\title{
DEGREE THEORY FOR EQUIVARIANT MAPS. I
}

\author{
J. IZE, I. MASSABÓ AND A. VIGNOLI
}

\begin{abstract}
A degree theory for equivariant maps is constructed in a simple geometrical way. This degree has all the basic properties of the usual degree theories and takes its values in the equivariant homotopy groups of spheres. For the case of a semifree $S^{1}$-action, a complete computation of these groups is given, the range of the equivariant degree is determined, and the general $S^{1}$. action is reduced to that special case. Among the applications one recovers and unifies both the degree for autonomous differential equations defined by Fuller [F] and the $S^{1}$-degree for gradient maps introduced by Dancer [Da]. Also, a simple but very useful formula of Nirenberg $[\mathrm{N}]$ is generalized (see Theorem 4.4(ii)).
\end{abstract}

\section{INTRODUCTION}

We expect this paper to be a reasonable example of the subject that we feel could be baptized as topological analysis. This term seems to us almost selfexplanatory. The gist would be to try to translate a problem from analysis into a problem in topology-or, to put it more gently, to try to use concepts and tools from topology in solving problems in analysis. Frequently, the nonvanishing of a topological invariant gives unexpected deep information on a certain question coming from analysis such as, say, the structure of the solution set of a given nonlinear equation. Thus, for example, ideas from singularity theory, Morse theory, Ljusternik-Snirel ' man category, index theories, topological degree theories, and their generalizations are more and more often used in analysis. This is particularly true with respect to what has been going on in nonlinear analysis during the past 15 years or so.

We believe that it is not necessary here to stress any further the topics covered by topological analysis and we hope that this label will be accepted by enough supporters among nonlinearists to become familiar in the mathematical community.

Our goal here is to carry on our program of research announced in [I.M.V] regarding the construction of a (generalized) degree theory for maps which are equivariant with respect to given representations of a compact Lie group $\Gamma$.

Received by the editors July 18, 1987 and, in revised form, April 14, 1988.

1980 Mathematics Subject Classification (1985 Revision). Primary 58B05, 58E07; Secondary 47H15, 54F45. degree.

Key words and phrases. Equivariant topological degree, $S^{1}$-homotopy groups of spheres, Fuller's 
In [I.M.V] we constructed a degree theory for $S^{1}$-equivariant maps defined on balls (see $\S 1$ for notation and definitions). We showed there that Fuller's degree [F] and the degree for $S^{1}$-equivariant gradient maps introduced in [Da] follow as particular cases. The techniques used in [I.M.V] originated mainly from elementary obstruction theory and we kept the spirit of some ideas already contained in [I].

We wish to present here both an extension and a simplification of what we have done in [I.M.V]. Namely, we extend our degree to maps defined on the closure of an arbitrary open set of the ambient space (not just on the closure of an open ball) and replace the circle group $S^{1}$ by an arbitrary compact Lie group $\Gamma$. Moreover, we simplify our presentation, avoiding obstruction theory. We shall rely instead on the very elementary idea of $\Gamma$-equivariant extensions of maps.

With a simple but useful trick we reduce the case of arbitrary domains to the case of maps defined on spheres. Consequently, our degree will be an element of the $\Gamma$-equivariant homotopy groups of spheres. These groups are by no means easy to compute. There are only some general results about their structure scattered in the literature. One of our tasks here is to compute them in the particular case when $\Gamma$ is the group $S^{1}$ acting almost semifreely. We shall be more specific on this topic when giving a more detailed report on the content of this paper.

We add in passing that our method of reducing the case of an arbitrary domain to that of spheres is interesting in itself even in the nonequivariant setting. Also, let us mention that a different approach of reducing an arbitrary domain to spheres has been presented in [G.M.V]. We briefly sketch it here so that the reader can compare both methods of reduction.

Let $U$ be an open and bounded subset of $\mathbf{R}^{m}$ and let $f: \bar{U} \rightarrow \mathbf{R}^{n}$ be a continuous map from the closure $\bar{U}$ of $U$ into $\mathbf{R}^{n}, n \leq m$, such that $f(x) \neq 0$ for any $x \in \partial U$. Now let $\mathbf{R}^{\wedge_{m}}, \mathbf{R}^{\wedge_{n}}$ be the Alexandroff onepoint compactification of $\mathbf{R}^{m}$ and $\mathbf{R}^{n}$ respectively and $\hat{f}: \mathbf{R}^{\wedge_{m}} \rightarrow \mathbf{R}^{\wedge_{n}}$ be a continuous extension of $f$ such that $\hat{f}(x)=f(x)$ for all $x \in \bar{U}$ and $\hat{f}(x) \neq$ 0 if $x \in \mathbf{R}^{\wedge_{m}} \backslash U$. Taking into account that $\mathbf{R}^{\wedge_{l}}$ is homeomorphic to the unit sphere $S^{l}$ of $\mathbf{R}^{l+1}$, one obtains a map $\tilde{f}: S^{m} \rightarrow S^{n}$ defined by $\tilde{f}=$ $\sigma_{n} \circ f \circ \sigma_{m}^{-1}$, where $\sigma_{n}: \mathbf{R}^{\wedge_{n}} \rightarrow S^{n}, \sigma_{m}: \mathbf{R}^{\wedge_{m}} \rightarrow S^{m}$ are the corresponding homeomorphisms. Thus, to any continuous map $f: \bar{U} \rightarrow \mathbf{R}^{n}$ not vanishing on $\partial U$ we may associate a continuous map $\tilde{f}: S^{m} \rightarrow S^{n}$. This device is exploited in [G.M.V] to define a generalized degree for maps acting between finite-dimensional Euclidean spaces of possibly different dimensions. When $m=n$ one recovers the classical Brouwer topological degree.

In the same situation our degree is constructed as follows. Let $B$ be a closed ball containing $U$ and let $\hat{f}: B \rightarrow \mathbf{R}^{n}$ be a continuous extension of $f$. Let $N$ be a bounded open neighborhood of $\partial U$ such that $\hat{f}(x) \neq 0$ for any $x \in \bar{N}$. Define $V=(U \cup N)^{c}$ and let $\varphi: B \rightarrow[0,1]$ be a Urysohn function such that 
$\varphi(x)=1$ if $x \in V$ and $\varphi(x)=0$ if $x \in \bar{U}$. Let $F:[0,1] \rightarrow \mathbf{R}^{n+1}$ be the map defined by

$$
F(t, x)=(2 t+2 \varphi(x)-1, \hat{f}(x)) .
$$

It is easy to see that $F(t, x)=0$ only if $x \in \Omega, f(x)=0$, and $t=\frac{1}{2}$. Thus $F$ maps $\partial([0,1] \times B)$ into $\mathbf{R}^{n+1} \backslash\{0\}$, which defines an element of $\pi_{m}\left(S^{n}\right)$, the generalized degree of $f$ with respect to $U$. The addition of two degrees is with respect to the group structure in $\pi_{m}\left(S^{n}\right)$. In the case when an action of a compact Lie group is present, this construction extends without difficulties.

An account of the structure of the present paper is in order. $\S 1$ is meant to make the whole work more handy and self-contained. It collects the majority of notions (to be used throughout the paper) which perhaps may not be too well known to nonspecialists.

The method we use in constructing our $\Gamma$-degree in infinite dimensions is strongly influenced by classical degree theories. Namely, we start with the construction of the degree for $\Gamma$-equivariant maps between finite-dimensional Euclidean spaces. This is carried out in $\S 2$ by reducing the case of arbitrary domains to that of spheres and showing that the $\Gamma$-degree is independent of this construction. We then prove its main properties which are analogous to those of the Brouwer topological degree. In particular, the Hopf classification property holds; i.e., if $f$ is defined on a ball $B$ and the $\Gamma$-degree of $f$ with respect to $B$ is trivial, then the restriction of $f$ to $\partial B$ has a nonvanishing $\Gamma$-equivariant extension to $B$. The additivity property is proved up to one suspension and we do not know if it holds in the case when the suspension is not performed.

The last result of $\S 2$ shows that when $\Gamma$ is the trivial group $\{e\}$ and $m=n$, then our $\Gamma$-degree reduces to the Brouwer topological degree.

We would like to point out some of the advantages of this definition, besides its quite simple geometric context: it is easy to construct and no heavy topological machinery is involved. Moreover, it is defined globally; that is, no generic arguments are used where one looks first at a local index and then proves that the degree obtained via the addition of the local indices is independent of the different approximations used (usually finite dimensional). Our construction is particularly handy in the case of equivariant problems since, in general, no generic framework is available. Furthermore, even in the case when one could define a local index for an isolated orbit, one has the problem of comparing the different "indices" for orbits of different type. It is also important to note that there are simple examples, coming from bifurcation theory, showing that a $\Gamma$-equivariant map has trivial generalized degree (forgetting the action) but its $\Gamma$-degree is nontrivial. This, together with some further observations on the $\Gamma$-degree, is given in Example D.7 of the Appendix.

In $\S 3$ we extend our theory to the infinite-dimensional context. In analogy with the classical case, we proceed by approximating a $\Gamma$-equivariant compact perturbation of the identity via $\Gamma$-equivariant maps acting in finite dimensions. 
Essentially, this can be done in two ways. The first way is sketched at the beginning of $\S 3$. The second one, which is closer in spirit to Leray and Schauder's construction [L.S], is embodied in Proposition 3.1. It should be emphasized again that this degree has all of the basic properties of a classical degree. Thus one may use it to obtain local and global results on existence, continuation, bifurcation, etc.

Let us stop for a moment and see what we have achieved so far. Essentially, we have shown that our $\Gamma$-degree is well defined as an element of the $\Gamma$-equivariant groups of spheres. We now face the formidable task of computing these groups. Since the corresponding computations are long and somewhat tedious we have decided to collect them in the Appendix. These results are exploited in $\S 4$ to show that Fuller's degree introduced in [F] and Dancer's degree [Da] follow as particular cases of our $S^{1}$-degree. We show, in particular, how to extend an $S^{1}$-equivariant map, not referring to obstruction theory, provided the action of $S^{1}$ is almost semifree. The fact that our $S^{1}$-degree does not depend on these extensions is by no means trivial and we settle this question in the long Theorem 4.2. The next step is to try to solve the problem of what elements of our $S^{1}$-homotopy groups of spheres are achieved by the $S^{1}$-degree. A complete answer is given by Theorem 4.3. Most of the proofs of the results contained in $\S 4$, due to their length and technicality, are given in Appendix D.

Let us say a few more words about the Appendix. We start by defining the $\Gamma$-homotopy groups of spheres $\pi_{m}^{\Gamma}\left(S^{n}\right)$ in a context which is suitable to our purposes. Next, we recall a $\Gamma$-equivariant version of the classical Freudenthal suspension theorem (see Theorem B). This is the main tool in extending the $\Gamma$-degree from the finite to the infinite-dimensional case.

Further, we collect some simple results on the structure of the group $\pi_{m}^{\Gamma}\left(S^{n}\right)$ (for example, Theorem C.2 represents a $\Gamma$-equivariant version of the classical Hopf classification theorem).

Finally, in D we confine ourselves to the particular case of an $S^{1}$-almost semifree action. In this case we are able to give complete information on the $S^{1}$-equivariant homotopy groups of spheres $\pi_{m}^{S^{1}}\left(S^{n}\right)$. In particular, we give explicit formulas for the generators of these groups.

In a forthcoming paper, which, as everyone would guess, will have the title Degree theory for equivariant maps, II, we shall undertake the study of our degree when the action is not necessarily almost semifree. This will involve the study of the degree with respect to all of the isotropy subgroups of $S^{1}$ (see Remark 2.1 in [I.M.V]).

\section{Preliminaries}

In this paper we shall use freely the following well-known facts and definitions regarding group actions and equivariant maps (cf. [B, Chapters 0 and 1]). The reader who is familiar with this material may skip 1.1 to 1.6. 
1.1. Group actions and the like. By a (left) topological transformation group we mean a triple $(\Gamma, X, \theta)$, where $\Gamma$ is a topological group, $X$ is a Hausdorff space, and $\theta: \Gamma \times X \rightarrow X$ is a continuous map satisfying the following conditions:

(i) $\theta\left(\gamma, \theta\left(\gamma^{\prime}, x\right)\right)=\theta\left(\gamma \gamma^{\prime}, x\right)$, for all $\gamma, \gamma^{\prime} \in \Gamma$ and all $x \in X$,

(ii) $\theta(e, x)=x$, for all $x \in X$, where $e$ is the identity of $\Gamma$.

The map $\theta$ is called the action of $\Gamma$ on $X$. The space $X$, together with a given action $\theta$ of $\Gamma$, is called a (left) $\Gamma$-space. In what follows we shall often use the notation $\theta(\gamma, x)=\gamma x$.

Let $X$ be a $\Gamma$-space and let $x \in X$. The set

$$
\Gamma_{x}=\{\gamma \in \Gamma: \gamma x=x\}
$$

is called the isotropy subgroup of $\Gamma$ at $x$. Obviously, $\Gamma_{x}$ is a closed subgroup of $\Gamma$, the action being continuous.

The action is said to be free if the (isotropy subgroup) $\Gamma_{x}$ is trivial (i.e., $\left.\Gamma_{x}=\{e\}\right)$ for any $x \in X$; thus for a free action each nontrivial element of $\Gamma$ moves every point of $X$. By a semifree action we mean that for each $x \in X$, $\Gamma_{x}$ is either trivial or is all of $\Gamma$.

A point $x \in X$ is called a fuxed point of $\Gamma$ on $X$, provided $\Gamma_{x}=\Gamma$.

We shall denote the subspace of fuxed points (fixed point space) of $\Gamma$ on $X$ by

$$
X^{\Gamma}=\{x \in X: \gamma x=x, \text { for all } \gamma \in \Gamma\} .
$$

(If $H$ is a subgroup of $\Gamma$, then $X^{H}$ will have the obvious meaning.)

Let $\Gamma$ and $X$ be as above and let $x \in X$. The subspace

$$
\Gamma(x)=\{\gamma x \in X: \gamma \in \Gamma\}
$$

is called the orbit of $x$ under $\Gamma$. It is easy to see that the orbits $\Gamma(x)$ and $\Gamma(y)$ of any two points $x, y \in X$ are either equal or disjoint. Let $X / \Gamma$ denote the set whose elements are the orbits $\hat{x}=\Gamma(x)$ of $\Gamma$ on $X$ (i.e., $\hat{x}=\hat{y}$ if and only if $x$ and $y$ are in the same orbit). Let $\pi: X \rightarrow X / \Gamma$ denote the natural map taking $x$ into its orbit $\hat{x}=\Gamma(x)$. Then the space $X / \Gamma$ endowed with the quotient topology (i.e., $U \subset X / \Gamma$ is open if and only if $\pi^{-1}(U)$ is open in $X$ ) is called the orbit space of $X$ with respect to $\Gamma$.

By a (real) representation of a topological group $\Gamma$ over a (real) Banach space $E$, we mean a homomorphism $\rho$ of $\Gamma$ into $\mathrm{GL}(E)$ - the general linear group of (linear) isomorphisms over $E$. This can be thought of as an action of $\Gamma$ on $E$ by linear transformations. In this case, we say that $\Gamma$ acts linearly on $E$ and the action $\theta: \Gamma \times E \rightarrow E$ is defined by $\theta(\gamma, x)=\rho(\gamma) x$; some authors prefer a terminology having a somewhat old-fashioned flavor: $\Gamma$ acts as a group of continuous linear operators on $E$; see, e.g; [R, p. 177].

1.2. Invariant sets and equivariant homotopies. A given subset $U$ of a $\Gamma$-space $X$ is called $\Gamma$-invariant if $\gamma x \in U$ for any $\gamma \in \Gamma$ and any $x \in U$ (i.e., $\Gamma(U) \subset U$ ). 
Let $X$ and $Y$ be $\Gamma$-spaces with actions $\theta$ and $\theta^{\prime}$ respectively, and let $U \subset$ $X$ be open and $\Gamma$-invariant. Then a map $g: U \rightarrow Y$ is called $\Gamma$-equivariant if

$$
g(\theta(\gamma, x))=\theta^{\prime}(\gamma, g(x)), \quad \text { for any } \gamma \in \Gamma \text { and } x \in U,
$$

and is called $\Gamma$-invariant if

$$
g(\theta(\gamma, x))=g(x), \quad \text { for any } \gamma \in \Gamma \text { and } x \in U .
$$

To simplify the writing, unless strictly necessary, we shall use the following notation:

$$
\begin{aligned}
& g(\gamma x)=\gamma^{\prime} g(x), \text { for } \Gamma \text {-equivariant maps, } \\
& g(\gamma x)=g(x), \text { for } \Gamma \text {-invariant maps } .
\end{aligned}
$$

It may be of interest to note that if $g$ is a $\Gamma$-equivariant $C^{1}$-map, then its Fréchet derivative $g_{x_{0}}$ at a stationary point $x_{0}$, that is, $\gamma x_{0}=x_{0}$ for any $\gamma \in \Gamma$, is also $\Gamma$-equivariant.

Two $\Gamma$-equivariant maps are $\Gamma$-homotopic if they are homotopic through $\Gamma$ equivariant maps. Alternatively, we define equivariant homotopies in terms of maps of cylinders. Namely, if $X$ is a $\Gamma$-space, we make $\Gamma$ act on $[0,1] \times X$ by

$$
\gamma(t, x)=(t, \gamma x), \quad \text { for any } \gamma \in \Gamma, t \in[0,1] \text {, and } x \in X .
$$

We shall say that the group $\Gamma$ acts trivially on $[0,1]$. Now two equivariant maps $f, g: X \rightarrow Y$ are $\Gamma$-homotopic, written $f \stackrel{\Gamma}{\sim} g$, if there exists a $\Gamma$-equivariant map $H:[0,1] \times X \rightarrow Y$ such that

$$
H(0, x)=f(x) \text { and } H(1, x)=g(x), \text { for all } x \in X .
$$

In the sequel we shall restrict our attention to the case when $\Gamma$ is a compact Lie group (i.e., $\Gamma$ is a compact topological group which is also a smooth manifold on which the group operations are smooth).

1.3. The Haar integral and isometric group representations. Let $E$ be a real Banach space and let $\Gamma$ be a compact Lie group acting linearly on $E$ with representation $\rho: \Gamma \rightarrow \mathrm{GL}(E)$.

One may renorm the Banach space $E$, for example by setting

$$
\|\mid x\|=\int_{\Gamma}\|\rho(\gamma) x\| d \gamma \quad \text { for } x \in E
$$

where the integral stands for the normalized Haar integral over the compact group $\Gamma$. Equipped with this new norm, the representation $\rho$ becomes an isometry due to the fact that the Haar integral is $\Gamma$-invariant on the class of continuous real-valued functions $f$ on $\Gamma$ under both left and right actions defined by $\left(L_{\gamma^{\prime}} f\right)(\gamma)=f\left(\left(\gamma^{\prime}\right)^{-1} \gamma\right)$ and $\left(R_{\gamma^{\prime}} f\right)(\gamma)=f\left(\gamma \gamma^{\prime}\right)$ respectively. This follows from

$$
\int_{\Gamma} f\left(\left(\gamma^{\prime}\right)^{-1} \gamma\right) d \gamma=\int_{\Gamma} f(\gamma) d \gamma=\int_{\Gamma} f\left(\gamma \gamma^{\prime}\right) d \gamma
$$

Thus, there is no loss of generality in assuming $\rho$ to be an isometry. 
1.4. Invariant Urysohn functions. Let $X$ be a normal space and let $\Gamma$ be a compact Lie group acting on $X$. Let $A$ and $B$ be closed $\Gamma$-invariant subsets of $X$ such that $A \cap B=\varnothing$.

Then there exists a continuous $\Gamma$-invariant Urysohn function $\varphi: X \rightarrow[0,1]$ such that $\varphi(x)=0$ if $x \in A$ and $\varphi(x)=1$ if $x \in B$. Indeed, let $\bar{\varphi}: X \rightarrow[0,1]$ be any Urysohn function relative to $A$ and $B$; then the required $\Gamma$-invariant Urysohn function is given by the standard averaging

$$
\varphi(x)=\int_{\Gamma} \bar{\varphi}(\gamma x) d \gamma .
$$

1.5. Construction of invariant neighborhoods. Let $X$ and $\Gamma$ be as above and let $A \subset X$ be a $\Gamma$-invariant closed set and $U \subset X$ be $\Gamma$-invariant, open, and such that $A \subset U$. Then there exists a $\Gamma$-invariant open subset $V$ such that $A \subset V \subset \bar{V} \subset U$. In fact, let $\varphi: X \rightarrow[0,1]$ be a $\Gamma$-invariant Urysohn function such that $\varphi(x)=0$ if $x \in A$ and $\varphi(x)=1$ if $x \in U^{c}$. A set with the required properties is given, for example, by $V=\varphi^{-1}\left(\left[0, \frac{1}{2}\right)\right)$.

1.6. The Dugundji-Gleason extension theorem. Let $\Gamma$ be a compact Lie group acting on a Banach space $E$ and let $A$ and $B$ be $\Gamma$-invariant closed subsets of $E$ such that $A \subset B$. Let $\rho: \Gamma \rightarrow \mathrm{GL}(F)$ be a representation of $\Gamma$ over a Banach space $F$ and let $g: A \rightarrow F$ be a $\Gamma$-equivariant map. Then there exists a $\Gamma$-equivariant extension $\bar{g}: B \rightarrow F$ of $g$. To see this, let $\hat{g}: B \rightarrow F$ be any Dugundji extension of $g$ (see [D]). The required $\Gamma$-equivariant extension is given by

$$
\bar{g}(x)=\int_{\Gamma} \rho\left(\gamma^{-1}\right) \hat{g}(\gamma x) d \gamma, \quad x \in B .
$$

Moreover, if $g$ is also compact, then so is $\bar{g}$. This follows by an argument given in [I, Lemma III.2.1].

1.7. Equivariant Borsuk homotopy extension theorem. The following statement is a direct extension to the equivariant context of a basic result in homotopy theory (see $[\mathrm{W}])$.

$$
\begin{aligned}
& \text { Let } \mathbf{R}^{m}, \mathbf{R}^{n} \text { be } \Gamma \text {-spaces and let } A, X \text { be closed } \Gamma \text {-invariant } \\
& \text { subsets of } \mathbf{R}^{m} \text { such that } A \subset X . \text { Let } F_{0}, F_{1}: A \rightarrow \mathbf{R}^{n} \backslash\{0\} \text { be } \\
& \Gamma \text {-equivariant maps which are } \Gamma \text {-homotopic. Then } F_{0} \text { extends } \\
& \Gamma \text {-equivariantly to } X \text { without zeros if and only if } F_{1} \text { does. }
\end{aligned}
$$

Indeed, let $\widetilde{F}_{0}$ be a $\Gamma$-equivariant extension of $F_{0}$ to $X$ and let $H: A \times$ $[0,1] \rightarrow \mathbf{R}^{n} \backslash\{0\}$ be a $\Gamma$-homotopy between $F_{0}$ and $F_{1}$.

Define $\bar{F}: X \times[0,1] \rightarrow \mathbf{R}^{n}$ to be any $\Gamma$-equivariant extension (where the group $\Gamma$ acts trivially on $[0,1])$ of the map

$$
F:(X \times\{0\}) \cup(A \times[0,1]) \rightarrow \mathbf{R}^{n} \backslash\{0\}
$$

defined by

$$
F(x, t)= \begin{cases}\widetilde{F}_{0}(x) & \text { if } x \in X \text { and } t=0 \\ H(x, t) & \text { if }(x, t) \in A \times[0,1]\end{cases}
$$


Let $B=\{x \in X: \bar{F}(x, t)=0$ for some $t \in[0,1]\}$. Clearly, $B$ is closed $\Gamma$-invariant and $A \cap B=\varnothing$. Hence there is a $\Gamma$-invariant Urysohn function $\varphi: X \rightarrow[0,1]$ such that $\varphi(B)=\{0\}$ and $\varphi(A)=\{1\}$.

Consider now the $\Gamma$-equivariant map $\widetilde{F}: X \times[0,1] \rightarrow \mathbf{R}^{n}$ defined by $\widetilde{F}(x, t)$ $=\bar{F}(x, \varphi(x) t)$. Notice that $\widetilde{F}(x, 0)=\widetilde{F}_{0}(x)$ for any $x \in X$ and $\widetilde{F}(x, 1)=$ $H(x, \varphi(x))$, if $x \in A$. Moreover, $\widetilde{F}(x, t) \neq 0$ for any $x \in X$ and any $t \in[0,1]$. Thus $\widetilde{F}(x, 1)$ is a nonvanishing $\Gamma$-equivariant extension of $F_{1}$ to $X$.

Note that $\widetilde{F}(x, 1)$ is $\Gamma$-homotopic to $\widetilde{F}_{0}(x)$, via $\widetilde{F}(x, t)$.

1.8. Equivariant suspension. Let us recall first the concept of suspension of a map in the case when there is no action.

For $k \in \mathbf{N}$, let $S^{k}$ denote the unit sphere in $\mathbf{R}^{k+1}$. Let $f: S^{m-1} \rightarrow S^{n-1}$ be a given map. The suspension $\Sigma(f): S^{m} \rightarrow S^{n}$ of $f$ is defined by first identifying $S^{m-1}$ with the equator of $S^{m}$ and similarly $S^{n-1}$ with $S^{n}$, then sending poles into poles, and finally extending $f$ linearly over meridians.

In particular, if

$$
g: S^{m} \rightarrow \mathbf{R}^{n+1} \backslash\{0\}
$$

is of the form

$$
g\left(x_{1}, \ldots, x_{m}, x_{m+1}\right)=\left(f\left(x_{1}, \ldots, x_{m}\right), x_{m+1}\right)
$$

then

$$
\Sigma(f)\left(x_{1}, \ldots, x_{m+1}\right)
$$

is homotopic to

$$
g\left(x_{1}, \ldots, x_{m+1}\right) /\left\|g\left(x_{1}, \ldots, x_{m+1}\right)\right\| .
$$

The homotopy classes of maps from $S^{m}$ into $S^{n}$ form the homotopy group $\pi_{m}\left(S^{n}\right)$. The important Freudenthal suspension theorem [S, p. 458] asserts that after a certain number of suspensions the homotopy class of a given map $f$ does not change any more. It is then called the stable homotopy class of $f$. More precisely, if $n$ is such that $k \leq n-2$ then the sequence of homomorphisms

$$
\pi_{k+n}\left(S^{n}\right) \stackrel{\Sigma}{\rightarrow} \pi_{k+n+1}\left(S^{n+1}\right) \stackrel{\Sigma}{\rightarrow} \cdots
$$

consists of isomorphisms. The direct limit of this sequence is called the $k$-stable homotopy group and denoted by $\Pi_{k}$.

In the case when $f: S^{m} \rightarrow S^{n}$ is $\Gamma$-equivariant, we may speak about the $\Gamma$-equivariant homotopy groups of spheres, denoted by

$$
\pi_{m}^{\Gamma}\left(S^{n}\right) \text {. }
$$

The fact that these sets are indeed groups (abelian under certain restrictions) is shown in Appendix A. For $\Gamma$-equivariant maps there is a corresponding notion of suspension. Given a $\Gamma$-space $V$ we denote by

$$
\Sigma^{V}(f): S^{m+\operatorname{dim} V} \rightarrow S^{n+\operatorname{dim} V}
$$


the $V$-Г-equivariant suspension of the $\Gamma$-equivariant map $f: S^{m} \rightarrow S^{n}$. In the case when $V=\mathbf{R}$ and $\Gamma$ acts trivially on $\mathbf{R}$, we shall use the notation $\Sigma(f)$ for the suspension of the $\Gamma$-equivariant map $f$. In this context an equivariant version of the Freudenthal suspension theorem is available (see $[H, N])$. The latter result is reported in Appendix B for the reader's convenience.

\section{GENERALIZED DEGREE FOR EQUIVARIANT MAPS IN FINITE DIMENSION}

In this section we construct a degree theory for $\Gamma$-equivariant maps acting on finite-dimensional $\Gamma$-spaces. We postpone to $\S 3$ the extension of this degree to the infinite-dimensional context.

Let $\Gamma$ be a compact Lie group acting linearly isometrically (see $\S 1$ ) on both $\mathbf{R}^{m}$ and $\mathbf{R}^{n}$. Let $\Omega$ be a $\Gamma$-invariant, bounded, open subset of $\mathbf{R}^{m}$ and let $f: \bar{\Omega} \rightarrow \mathbf{R}^{n}$ be a $\Gamma$-equivariant map such that

$$
f(x) \neq 0 \text { for } x \in \partial \Omega \text {. }
$$

We construct our degree as follows. Consider a closed ball $B_{R}$ centered at the origin with radius $R$ such that $\Omega \subset B_{R}$ (notice that $B_{R}$ is $\Gamma$-invariant since $\Gamma$ acts isometrically). Now let $\hat{f}: B_{R} \rightarrow \mathbf{R}^{n}$ be a continuous $\Gamma$-equivariant extension of $f$ such that $\hat{f}(x) \neq 0$ for any $x \in \bar{N}$, where $N$ is a $\Gamma$-invariant, bounded, open neighborhood of $\partial \Omega$ with $\bar{N} \subset B_{R}$. The existence of such a neighborhood is ensured both by the continuity of $f$ and by $\S 1.5$ of the Preliminaries. Define $V=(\Omega \cup N)^{c}$. Clearly, $V$ is $\Gamma$-invariant closed and $V \cap \bar{\Omega}=\varnothing$.

Let $\varphi: B_{R} \rightarrow[0,1]$ be a $\Gamma$-invariant Urysohn function such that $\varphi(x)=1$ if $x \in V$ and $\varphi(x)=0$ if $x \in \bar{\Omega}$. Let $F:[0,1] \times B_{R} \rightarrow \mathbf{R}^{n+1}$ be the $\Gamma$ equivariant map defined by

$$
F(t, x)=(2 t+2 \varphi(x)-1, \hat{f}(x))
$$

where $\Gamma$ acts trivially both on $[0,1]$ and on the first component of $\mathbf{R}^{n+1}$.

Notice that if $x \notin \bar{\Omega} \cup \bar{N}$ then $F(t, x)=(2 t+1, \hat{f}(x)), t \in[0,1]$, and if $x \in \bar{\Omega}$ then $F(t, x)=(2 t-1, \hat{f}(x)), t \in[0,1]$. Moreover, $F(t, x) \neq 0$ for any $x \in \Omega^{c} \cup \bar{N}, t \in[0,1]$. Hence if $F(t, x)=0$, then $x \in \Omega, \hat{f}(x)=f(x)=$ 0 , and $t=\frac{1}{2}$. Now $F$ maps $\partial\left([0,1] \times B_{R}\right)$ into $\mathbf{R}^{n+1} \backslash\{0\}$ and therefore the restriction of $F$ to $\partial\left([0,1] \times B_{R}\right)$ can be thought of as a $\Gamma$-equivariant map from $S^{m}$ into $S^{n}$ via the standard identifications $\partial\left([0,1] \times B_{R}\right) \approx S^{m}$ and $\mathbf{R}^{n+1} \backslash\{0\} \approx S^{n}$, respectively. We may thus consider the $\Gamma$-equivariant homotopy class $[F]_{\Gamma}$ of $F$ as an element of the $\Gamma$-equivariant homotopy group $\pi_{m}^{\Gamma}\left(S^{n}\right)$ (see Appendix A).

At this stage some readers may be puzzled by our somewhat tricky construction of the degree. Why not extend the $\Gamma$-equivariant map $f: \bar{\Omega} \rightarrow \mathbf{R}^{n}$ directly to a $\Gamma$-equivariant map $\hat{f}: B_{R} \rightarrow \mathbf{R}^{n}$ ? The point is, of course, that the extension $\hat{f}$ may vanish on $B_{R} \backslash \Omega$ (see also Remark 2.4 below). 
We have seen that to any map $f$ as above there corresponds an element $[F]_{\Gamma} \in \pi_{m}^{\Gamma}\left(S^{n}\right)$. Our next goal is to show that this correspondence is independent of its construction. Namely, we have the following:

Proposition 2.1. The homotopy class $[F]_{\Gamma}$ does not depend on

(a) the $\Gamma$-invariant Urysohn function $\varphi$,

(b) the choice of the $\Gamma$-invariant neighborhood $N$ of $\partial \Omega$,

(c) the $\Gamma$-equivariant extension $\hat{f}$ of $f$,

(d) the choice of the ball $B_{R}$ containing $\bar{\Omega}$.

Proof. (a) Let $\varphi_{0}, \varphi_{1}: B_{R} \rightarrow[0,1]$ be two $\Gamma$-invariant Urysohn functions vanishing on $\bar{\Omega}$ and taking value 1 on $V=(\Omega \cup N)^{c}$. Consider the map $\varphi_{\tau}: B_{R} \rightarrow[0,1], \tau \in[0,1]$, defined as $\varphi_{\tau}(x)=\tau \varphi_{1}(x)+(1-\tau) \varphi_{0}(x), x \in B_{R}$ and $\tau \in[0,1]$. Let $F_{\tau}:[0,1] \times B_{R} \rightarrow \mathbf{R}^{n+1}, \tau \in[0,1]$, be defined by

$$
F_{\tau}(t, x)=\left(2 t+2 \varphi_{\tau}(x)-1, \hat{f}(x)\right) \text {. }
$$

Clearly, $F_{\tau}(t, x)=(2 t+1, \hat{f}(x))$ for any $x \in \partial B_{R}$ and $t \in[0,1]$.

Moreover,

$$
F_{\tau}(0, x)=\left\{\begin{array}{ll}
(1, \hat{f}(x)), \\
\neq 0, \\
(-1, \hat{f}(x)),
\end{array} \quad F_{\tau}(1, x)= \begin{cases}(3, \hat{f}(x)) & \text { if } x \notin \bar{\Omega} \cup \bar{N}, \\
\neq 0 & \text { if } x \in \Omega^{c} \cap N, \\
(+1, \hat{f}(x)) & \text { if } x \in \bar{\Omega},\end{cases}\right.
$$

for any $\tau \in[0,1]$. Hence $F_{\tau}$ is an admissible $\Gamma$-homotopy between $F_{0}$ and $F_{1}$. Therefore $\left[F_{0}\right]_{\Gamma}=\left[F_{1}\right]_{\Gamma}$; that is, the $\Gamma$-homotopy class of $F$ does not depend on the choice of the Urysohn function.

(b) Let us assume first that there exist two invariant open neighborhoods $N_{0}$, $N_{1}$ of $\partial \Omega$ such that $N_{0} \subset N_{1} \subset B_{R}$. Let $\varphi_{0}, \varphi_{1}$ be the $\Gamma$-invariant Urysohn functions associated to $N_{0}, N_{1}$ respectively. Consider the map $\varphi_{\tau}(x)=$ $\tau \varphi_{1}(x)+(1-\tau) \varphi_{0}(x)$, for $x \in B_{R}, \tau \in[0,1]$, and the admissible $\Gamma$-homotopy $F_{\tau}:[0,1] \times B_{R} \rightarrow \mathbf{R}^{n+1}, \tau \in[0,1]$, between $F_{0}$ and $F_{1}$ which acts only on the first coordinate

$$
F_{\tau}(t, x)=\left(2 t+2 \varphi_{\tau}(x)-1, \hat{f}(x)\right) .
$$

This gives immediately that $\left[F_{0}\right]_{\Gamma}=\left[F_{1}\right]_{\Gamma}$.

In the case when $N_{0}, N_{1}$ are arbitrary, one can use the previous argument applied to $N_{0} \cap N_{1}$ and to each $N_{0}$ and $N_{1}$.

(c) Given two $\Gamma$-equivariant extensions $\hat{f}_{0}, \hat{f}_{1}$ of $f$ we can choose a $\Gamma$ invariant open neighborhood $N$ of $\partial \Omega$ on which the $\Gamma$-equivariant map $\tau \hat{f}_{1}+$ $(1-\tau) \hat{f}_{0}$ is not vanishing for $\tau \in[0,1]$. This map induces an admissible $\Gamma$-homotopy $F_{\tau}$ via (2.1) and the assertion follows.

(d) Let $B_{R_{0}} \subset B_{R}, 0 \leq R_{0} \leq R$, be such that $\Omega \subset B_{R_{0}}$. Let $\hat{f}_{0}$ and $\hat{f}$ be two $\Gamma$-equivariant extensions of $f$ to $B_{R_{0}}$ and $B_{R}$, respectively. By (b) and (c) we may assume that $\hat{f}_{0}$ and $\hat{f}$ do not vanish on a common $\Gamma$-invariant 
open neighborhood $N \subset B_{R_{0}}$ of $\partial \Omega$ and are such that $\hat{f}_{\mid B_{R_{0}}}=\hat{f}_{0}$. Let $\varepsilon>0$ be such that if $x \in \bar{N}$ then $\|x\| \leq R_{0}-\varepsilon$. Consider the $\Gamma$-map

$$
\hat{h}_{\tau}(x)=\hat{f}(\alpha(\tau, x) x) / \alpha(\tau, x), \quad \text { for any } \tau \in[0,1],
$$

where

$$
\alpha(\tau, x)= \begin{cases}1 & \text { if }\|x\| \leq R_{0}-\varepsilon, \\ 1+\tau \frac{\left(R-R_{0}\right)\left(\|x\|-R_{0}+\varepsilon\right)}{\varepsilon R_{0}} & \text { if } R_{0}-\varepsilon \leq\|x\| \leq R_{0} .\end{cases}
$$

For any $\tau \in[0,1]$, the scaling $S_{\tau}(x):=\alpha(\tau, x) x$ is a $\Gamma$-equivariant homeomorphism from $B_{R_{0}}$ into $B_{R}$, leaving fixed $B_{R_{0}-\varepsilon}$ and $S_{1}\left(B_{R_{0}}\right)=B_{R}$. Hence, $\hat{h}_{\tau}$ is a $\Gamma$-equivariant extension of $f$ to $B_{R_{0}}$ for any $\tau \in[0,1]$.

Thus, by (c), since $\hat{h}_{0}=\hat{f}_{\mid B_{R_{0}}}=\hat{f}_{0}$, the $\Gamma$-homotopy class $\left[F_{0}\right]_{\Gamma}$ of $F_{0}$ induced by $\hat{f}_{0}$ coincides with the class $\left[F_{1}\right]_{\Gamma}$, where $F_{1}$ is induced by $\hat{h}_{1}$, via (2.1).

Moreover, if we extend $\hat{h}_{1}$ as a constant outside $B_{R_{0}}$, we obtain a $\Gamma$ equivariant extension $\hat{h}_{1}$ of $f$ to $B_{R}$. Thus, once again applying (c) we have that $\left[F_{1}\right]_{\Gamma}=[F]_{\Gamma}$, where $F_{1}, F$ are the maps induced by $\hat{f}_{1}, f$ respectively. (Here we identify, via the scaling, the two groups of $\Gamma$-homotopy classes of maps defined on the two cylinders $[0,1] \times B_{R_{0}}$ and $[0,1] \times B_{R^{*}}$ ) Q.E.D.

We are now in a position to introduce the following important

Definition 2.2. To each $\Gamma$-equivariant map $f: \bar{\Omega} \rightarrow \mathbf{R}^{n}$, not vanishing on $\partial \Omega$, we assign the element $[F]_{\Gamma} \in \pi_{m}^{\Gamma}\left(S^{n}\right)$ and we call it the generalized $\Gamma$-degree of $f$, denoted by $\operatorname{deg}_{\Gamma}(f, \Omega)$.

We now list the main properties of this degree.

(a) Existence property. If $\operatorname{deg}_{\Gamma}(f, \Omega)$ is nontrivial, then there exists $x \in \Omega$ such that $f(x)=0$.

The proof of (a) can be drawn out of Remark A.2 in the Appendix. Note, however, that the $\Gamma$-equivariance of $f$ implies $f(\gamma x)=0$ for all $\gamma \in \Gamma$; that is, solutions come by orbits.

(b) $\Gamma$-homotopy invariance property. Let $f_{\tau}: \bar{\Omega} \rightarrow \mathbf{R}^{n}, 0 \leq \tau \leq 1$, be a continuous one-parameter family of $\Gamma$-equivariant maps not vanishing on $\partial \Omega$ for all $\tau \in[0,1]$. Then the generalized $\Gamma$-degree $\operatorname{deg}_{\Gamma}\left(f_{\tau}, \Omega\right)$ does not depend on $\tau \in[0,1]$.

Proof. Immediate from the fact that the construction of $F_{\tau}:[0,1] \times B_{R} \rightarrow \mathbf{R}^{n+1}$ via (2.1) can be performed uniformly with respect to $\tau$. Q.E.D.

(c) Excision property. Let $f: \bar{\Omega} \rightarrow \mathbf{R}^{n}$ be a continuous $\Gamma$-equivariant map such that $f(x) \neq 0$ in $\bar{\Omega} \backslash \Omega_{0}$, where $\Omega_{0} \subset \Omega$ is open and $\Gamma$-invariant. Then

$$
\operatorname{deg}_{\Gamma}(f, \Omega)=\operatorname{deg}_{\Gamma}\left(f_{\mid \bar{\Omega}_{0}}, \Omega_{0}\right) .
$$


Proof. Let $\Omega \subset B_{R}$, let $\hat{f}: B_{R} \rightarrow \mathbf{R}^{n}$ be a $\Gamma$-equivariant extension of $f$ to $B_{R}$, let $N$ be a $\Gamma$-invariant open neighborhood of $\partial \Omega$ where $\hat{f}$ does not vanish, and let $\varphi: B_{R} \rightarrow[0,1]$ be a $\Gamma$-invariant Urysohn function associated to $\bar{N}$; that is, $\varphi(x)=0$ if $x \in \bar{\Omega}$ and $\varphi(x)=1$ if $x \notin \Omega \cup N$. Thus $\operatorname{deg}_{\Gamma}(f, \Omega)=[F]_{\Gamma}$, where $F$ is defined by (2.1).

Now observe that the map $\hat{f}$ is also an extension of the restriction $f_{\mid \bar{\Omega}_{0}}$ of $f$ to $\bar{\Omega}_{0}$ which never vanishes on the $\Gamma$-invariant neighborhood $\bar{N}^{\prime}=$ $\left(\bar{\Omega} \backslash \Omega_{0}\right) \cup \bar{N} \cup\left(\bar{\Omega} \cap \bar{N}_{0}\right)$ of $\partial \Omega_{0}$ (where $N_{0}$ is the neighborhood of $\left.\partial \Omega_{0}\right)$.

Since $\bar{\Omega}_{0} \cup \bar{N}^{\prime}=\bar{\Omega} \cup \bar{N}$, the Urysohn function $\varphi$ is also a Urysohn function associated to $\bar{N}^{\prime}$. Thus, being $\operatorname{deg}_{\Gamma}\left(f_{\left.\right|_{\Omega_{0}}}, \Omega_{0}\right)$ independent of the choice of the neighborhood of $\partial \Omega_{0}$ and of the Urysohn function, it follows that

$$
[F]_{\Gamma}=\operatorname{deg}_{\Gamma}\left(f_{\mid \bar{\Omega}_{0}}, \Omega_{0}\right) .
$$

In particular, if $f(x) \neq 0$ for all $x \in \bar{\Omega}$, then $\operatorname{deg}_{\Gamma}(f, \Omega)=0$ (take $\Omega_{0}=$ $\phi)$. Q.E.D.

Remark 2.3. Using the excision property we may extend the notion of $\Gamma$-degree to the class of $\Gamma$-equivariant maps $f: \Omega \rightarrow \mathbf{R}^{n}, \Omega$ an open (not necessarily bounded) $\Gamma$-invariant set of $\mathbf{R}^{m}$, such that $f^{-1}(0)$ is a compact set, by restricting $f$ to a bounded open $\Gamma$-invariant set $\Omega_{0} \supset f^{-1}(0)$.

(d) Suspension property. Let $f: B_{R} \rightarrow \mathbf{R}^{n}$ be an $\Gamma$-equivariant continuous map not vanishing on $\partial B_{R}$. Then

$$
\operatorname{deg}_{\Gamma}\left(f, B_{R}\right)=\Sigma\left([f]_{\Gamma}\right) .
$$

Proof. Using a nonvanishing radial extension of $f$, we have that

$$
\operatorname{deg}_{\Gamma}\left(f, B_{R}\right)=[F]_{\Gamma}=[2 t-1, f]_{\Gamma}=\Sigma\left([f]_{\Gamma}\right) .
$$

Here $[f]_{\Gamma} \in \pi_{m-1}^{\Gamma}\left(S^{n-1}\right)$ and $\Sigma$ stands for the (one-dimensional) suspension homomorphism (see §1.8). Q.E.D.

Remark 2.4. Let $f: \bar{\Omega} \rightarrow \mathbf{R}^{n}$ be a $\Gamma$-equivariant continuous map such that $f(x) \neq 0$ for all $x \in \partial \Omega$ and assume that there exists a $\Gamma$-equivariant extension $\hat{f}: B_{R} \rightarrow \mathbf{R}^{n}$ of $f$ to $B_{R} \supset \Omega$ such that $\hat{f}(x) \neq 0$ for any $x \in B_{R} \backslash \Omega$. Then one may consider the $\Gamma$-homotopy class $[\hat{f}]_{\Gamma}$ in $\pi_{m-1}^{\Gamma}\left(S^{n-1}\right)$ as well the $\Gamma$ homotopy class in $\pi_{m}^{\Gamma}\left(S^{n}\right)$ of the map $F$ defined by $(2.1)$, that is, $\operatorname{deg}_{\Gamma}\left(\hat{f}, B_{R}\right)$. In this case, using the suspension and the excision properties of the $\Gamma$-degree, one obtains

$$
\operatorname{deg}_{\Gamma}\left(\hat{f}, B_{R}\right)=\Sigma\left([\hat{f}]_{\Gamma}\right)=\operatorname{deg}_{\Gamma}(f, \Omega) .
$$

We are now ready for the following

Corollary 2.5. Let $f: \bar{\Omega} \rightarrow \mathbf{R}^{n}$ be a continuous $\Gamma$-equivariant map such that $f(x) \neq 0$ for all $x \in \partial \Omega$. Let $\hat{f}_{i}: B_{R} \rightarrow \mathbf{R}^{n}, \Omega \subset B_{R} \subset \mathbf{R}^{m}, i=0,1$, be $\Gamma$-equivariant extensions of $f$ to $B_{R}$ (possibly with zeros in $B_{R} \backslash \Omega$ ) and let 
$g_{i}:[0,1] \times B_{R} \rightarrow \mathbf{R}, i=0,1$, be continuous $\Gamma$-invariant functions. Assume further that the maps $F_{i}:[0,1] \times B_{R} \rightarrow \mathbf{R}^{n+1}, i=0,1$, defined by

$$
F_{i}(t, x)=\left(g_{i}(t, x), \hat{f}_{i}(x)\right), \quad i=0,1,
$$

are $\Gamma$-homotopic on $\partial([0,1] \times \bar{\Omega})$ and such that

$$
F_{i}(t, x) \neq 0 \text { for all }(t, x) \in[0,1] \times\left(B_{R} \backslash \Omega\right) .
$$

Then

$$
\Sigma\left(\left[F_{0}\right]_{\Gamma}\right)=\Sigma\left(\left[F_{1}\right]_{\Gamma}\right) .
$$

Proof. Since $\operatorname{deg}_{\Gamma}\left(F_{0},(0,1) \times \Omega\right)=\operatorname{deg}_{\Gamma}\left(F_{1},(0,1) \times \Omega\right)$, then applying (2.2) to $\Omega^{\prime}=(0,1) \times \Omega$ and $F_{0}, F_{1}$, respectively, we get the assertion. Q.E.D.

(e) Additivity property (up to one suspension). Let $f: \bar{\Omega} \rightarrow \mathbf{R}^{n}$ be a $\Gamma$-equivariant continuous map such that $f(x) \neq 0$ on $\partial \Omega$ and let $\Omega=\Omega_{1} \cup \Omega_{2}$, where $\Omega_{1}, \Omega_{2}$ are open $\Gamma$-invariant subsets of $\Omega$ such that $\bar{\Omega}_{1} \cap \bar{\Omega}_{2}=\varnothing$.

Then

$$
\Sigma\left([F]_{\Gamma}\right)=\Sigma\left(\left[F_{1}\right]_{\Gamma}\right)+\Sigma\left(\left[F_{2}\right]_{\Gamma}\right),
$$

where $[F]_{\Gamma},\left[F_{1}\right]_{\Gamma}$, and $\left[F_{2}\right]_{\Gamma}$ are the $\Gamma$-equivariant homotopy classes induced by $f, f_{\bar{\Omega}_{1}}$, and $f_{\mid \bar{\Omega}_{2}}$ respectively.

Proof. Let $[F]_{\Gamma}$ be the $\Gamma$-equivariant homotopy class induced via the map $F:[0,1] \times B_{R} \rightarrow \mathbf{R}^{n+1}$ defined by

$$
F(t, x)=(2 t+2 \varphi(x)-1, \hat{f}(x))
$$

where $\varphi$ is a $\Gamma$-invariant Urysohn function associated to an open, bounded, $\Gamma$-invariant neighborhood $N$ of $\partial \Omega$ (on which $\hat{f} \neq 0$ ) such that $N=N_{1} \cup$ $N_{2}$ and $\bar{N}_{1} \cap \bar{N}_{2}=\varnothing$, where $N_{1}$ and $N_{2}$ are the corresponding $\Gamma$-invariant neighborhoods of $\partial \Omega_{1}$ and $\partial \Omega_{2}$ respectively. We shall denote by $\varphi_{1}$ and $\varphi_{2}$ the Urysohn functions associated to $N_{1}$ and $N_{2}$ respectively. Consider the following $\Gamma$-homotopy, $\tau \in[0,1]$ :

$$
\begin{cases}(2 t+(1-2 t \tau)(2 \varphi(x)-1), \hat{f}(x)), & 0 \leq t \leq \frac{1}{2}, \\ ((1-\tau)(2 t+2 \varphi(x)-1)+\tau, \hat{f}(x)), & \frac{1}{2} \leq t \leq 1 .\end{cases}
$$

Setting $\tau=1$, we obtain that $[F]_{\Gamma}$ is induced by the map

$$
\begin{cases}(2 t+(1-2 t)(2 \varphi(x)-1), \hat{f}(x)), & 0 \leq t \leq \frac{1}{2} \\ (1, \hat{f}(x)), & \frac{1}{2} \leq t \leq 1 .\end{cases}
$$

Notice that if we change the variable $t$ to $1-t$ in the above map, the new map so obtained induces the class $-[F]_{\Gamma}$ (the inverse of $[F]_{\Gamma}$; see Appendix A).

Consider the map

$$
\begin{cases}(2 t+(1-2 t)(2 \varphi(x)-1), \hat{f}(x)), & 0 \leq t \leq \frac{1}{2} \\ \left(2(1-t)+(2 t-1)\left(2 \varphi_{1}(x)-1\right), \hat{f}(x)\right), & \frac{1}{2} \leq t \leq 1 .\end{cases}
$$


This is the map which induces $[F]_{\Gamma}-\left[F_{1}\right]_{\Gamma}$. Next, consider the $\Gamma$-homotopy $H_{\tau}:[0,1] \times B_{R} \rightarrow \mathbf{R}^{n+1}$ defined by

$$
\begin{gathered}
H_{\tau}(t, x)=(1, \hat{f}(x)) \quad \text { if } t \in[0,1], x \notin \bar{\Omega} \cup \bar{N}, \text { and } \tau \in[0,1] ; \\
H_{\tau}(t, x)=\left\{\begin{array}{c}
\left(2 t+(1-2 t)\left(2 \varphi_{1}(x)-1\right), \hat{f}(x)\right) \quad \text { if } 0 \leq 2 t \leq \tau \text { and } x \in \bar{\Omega}_{1} \cup \bar{N}_{1}, \\
\left(\tau+(1-\tau)\left(2 \varphi_{1}(x)-1\right), \hat{f}(x)\right) \\
\text { if } \tau \leq 2 t \leq 2-\tau \text { and } x \in \bar{\Omega}_{1} \cup \bar{N}_{1}, \\
\left(2(1-t)+(2 t-1)\left(2 \varphi_{1}(x)-1\right), \hat{f}(x)\right) \\
\text { if } 2-\tau \leq 2 t \leq 2 \text { and } x \in \bar{\Omega}_{1} \cup \bar{N}_{1} ;
\end{array}\right. \\
H_{\tau}(t, x)=\left\{\begin{array}{c}
\left(2 t+(1-2 t)\left(2 \varphi_{2}(x)-1\right), \hat{f}(x)\right) \quad \text { if } 0 \leq t \leq \frac{1}{2} \text { and } x \in \bar{\Omega}_{2} \cup \bar{N}_{2}, \\
(1, \hat{f}(x)) \quad \text { if } \frac{1}{2} \leq t \leq 1 \text { and } x \in \bar{\Omega}_{2} \cup \bar{N}_{2} .
\end{array}\right.
\end{gathered}
$$

It is easy to check that $H_{\tau}$ is well defined (recall that $\varphi_{\mid \bar{\Omega}_{i} \cup \bar{N}_{i}}=\varphi_{i}, i=1,2$ ) and continuous. Clearly $H_{1}$ coincides with the map defined by (2.3). On the other hand,

$$
H_{0}(t, x)=\left(2 \varphi_{1}(x)-1, \hat{f}(x)\right) \quad \text { if } t \in[0,1], x \in \bar{\Omega}_{1} \cup \bar{N}_{1}
$$

and the restriction of $H_{0}$ to $[0,1] \times\left(\bar{\Omega}_{2} \cup \bar{N}_{2}\right)$ coincides with $F_{2}$. Thus the map $H_{0}$ can be viewed as an extension of $F_{2}$ which is nonvanishing on

$$
[0,1] \times B_{R} \backslash\left(\bar{\Omega}_{2} \cup \bar{N}_{2}\right) .
$$

Therefore, by Corollary 2.5 , we obtain $\Sigma\left([F]_{\Gamma}-\left[F_{1}\right]_{\Gamma}\right)=\Sigma\left(\left[F_{2}\right]_{\Gamma}\right)$. Since $\Sigma$ is a homomorphism we are done. Q.E.D.

Remark 2.6. In the case when $\Omega_{2}=\varnothing$, taking $\varphi_{2}=1$ in the above argument, one gets that $[F]_{\Gamma}-\left[F_{1}\right]_{\Gamma}$ is represented by the map

$$
\left(2 \varphi_{1}(x)-1, \hat{f}(x)\right) .
$$

This map is nonvanishing on the whole of $[0,1] \times B_{R}$, hence homotopically trivial on the boundary of the cylinder, that is, $[F]_{\Gamma}-\left[F_{1}\right]_{\Gamma}=0_{\Gamma}$ (this is another way of computing the class $-[F]_{\Gamma}$, which is the inverse of $[F]_{\Gamma}$ ).

Remark 2.7. Note that in all the above homotopies one has the last component $\hat{f}(x)$ unchanged. Thus one can show that the additivity property also holds in infinite dimensions when compact perturbations of the identity are considered.

(f) Relation between the generalized $\Gamma$-degree and $\Gamma$-epi maps. The class of $\Gamma$ epi maps has been introduced in [I.M.V]. The definition runs as follows (adapted here to the case of bounded domains).

A continuous $\Gamma$-equivariant map $f: \bar{\Omega} \rightarrow \mathbf{R}^{n}$ is called $\Gamma$-epi provided that (a) $f(x) \neq 0$ for all $x \in \partial \Omega$, and (b) for any continuous $\Gamma$-equivariant map $h: \bar{\Omega} \rightarrow \mathbf{R}^{n}$ such that $h(x)=0$ for all $x \in \partial \Omega$, the equation $f(x)=h(x)$ has a solution $x \in \Omega$. Let us add in passing that this class of maps coincides with 
that of $\Gamma$-essential maps (i.e., $f$ does not admit nonvanishing $\Gamma$-equivariant extensions $\bar{f}$ from $\partial \Omega$ to $\bar{\Omega}$ ). In this context the following relation holds.

Let $f: \bar{\Omega} \rightarrow \mathbf{R}^{n}$ be such that $\operatorname{deg}_{\Gamma}(f, \Omega)$ is not trivial; then $f$ is $\Gamma$-epi ( $\Gamma$-essential).

This follows at once from

$$
\operatorname{deg}_{\Gamma}(f-h, \Omega)=\operatorname{deg}_{\Gamma}(f, \Omega)
$$

provided $h$ is continuous $\Gamma$-equivariant and vanishing on $\partial \Omega$. This is, in its turn, a consequence of the boundary dependence property of the generalized $\Gamma$-degree: let $f, g: \bar{\Omega} \rightarrow \mathbf{R}^{n}$ be $\Gamma$-equivariant maps such that

$$
f(x)=g(x) \text { for all } x \in \partial \Omega \text {; }
$$

then, if defined, $\operatorname{deg}_{\Gamma}(f, \Omega)=\operatorname{deg}_{\Gamma}(g, \Omega)$. This last equality is an immediate consequence of the homotopy property and the fact that, under the above assumption, the maps $f$ and $g$ are $\Gamma$-homotopic.

(g) Recovering the Brouwer topological degree. In the case when $\Gamma=\{e\}$ (i.e., $\Gamma$ is the trivial group) and $m=n$ the generalized $\Gamma$-degree introduced above reduces to the classical Brouwer topological degree (here denoted by $\operatorname{deg}_{B}$ ). This can be shown as follows:

$$
\begin{aligned}
\operatorname{deg}_{\Gamma}(f, \Omega) & =[F]=\operatorname{deg}_{B}\left(F,(0,1) \times B_{R}, 0\right)=\operatorname{deg}_{B}(F,(0,1) \times \Omega, 0) \\
& =\operatorname{deg}_{B}((2 t-1, f),(0,1) \times \Omega, 0) .
\end{aligned}
$$

Now, by the product formula of the Brouwer degree, we obtain

$$
\begin{aligned}
\operatorname{deg}_{B}((2 t-1, f),(0,1) \times \Omega, 0) & =\operatorname{deg}_{B}(2 t-1,(0,1), 0) \operatorname{deg}_{B}(f, \Omega, 0) \\
& =\operatorname{deg}_{B}(f, \Omega, 0) .
\end{aligned}
$$

If $m \neq n$, then the same relationship holds with cohomotopy theory (cf. $\left[\mathrm{I}_{0}\right]$ ) except that our addition is a homotopy operation and not a cohomotopy sum.

\section{GENERALIZED DEGREE FOR EQUIVARIANT MAPS}

IN INFINITE DIMENSIONS AND FINITE-DIMENSIONAL APPROXIMATIONS

In this section we extend our $\Gamma$-degree to the context of infinite-dimensional Banach spaces and then show how to compute it by means of finite-dimensional approximations. We shall confine ourselves to the following case, which is particularly suitable for applications.

Let $E$ be a Banach space and let $\Gamma$ be a compact Lie group acting linearly isometrically on each of the spaces $E, \mathbf{R}^{M}$, and $\mathbf{R}^{N}, M, N \in \mathbf{N}$. Let $\Omega$ be a $\Gamma$-invariant open bounded subset of $\mathbf{R}^{M} \times E$ and let $f: \bar{\Omega} \rightarrow \mathbf{R}^{N} \times E$ be a compact $\Gamma$-equivariant map of the form

$$
f(x, y)=\left(f_{N}(x, y), f_{\infty}(x, y)\right) \in \mathbf{R}^{N} \times E, \quad \text { for }(x, y) \in \bar{\Omega} \subset \mathbf{R}^{M} \times E .
$$


Finally, let us denote by $\Phi: \bar{\Omega} \rightarrow \mathbf{R}^{N} \times E$ the $\Gamma$-equivariant map defined as

$$
\Phi(z)=\left(-f_{N}(x, y), y-f_{\infty}(x, y)\right), \quad \text { where } z=(x, y) \in \bar{\Omega} .
$$

To define a $\Gamma$-degree for the map $\Phi$ with respect to the set $\Omega$ we make the basic assumption

$$
\Phi(z) \neq 0 \text { for all } z \in \partial \Omega \text {. }
$$

Mimicking the construction of the $\Gamma$-degree in finite-dimensional spaces, we consider first a ball $B$ centered at the origin containing $\Omega$ and a compact $\Gamma$ equivariant extension $\hat{f}=\left(\hat{f}_{N}, \hat{f}_{\infty}\right)$, of the map $f=\left(f_{N}, f_{\infty}\right)$ to $B$. We then proceed as follows.

Let $\widehat{\Phi}: B \rightarrow \mathbf{R}^{N} \times E$ be the $\Gamma$-equivariant map defined by

$$
\widehat{\Phi}(z)=\left(-\hat{f}_{N}(x, y), y-\hat{f}_{\infty}(x, y)\right)=y-\hat{f}(x, y), \quad z=(x, y) \in B \subset \mathbf{R}^{M} \times E .
$$

Now, using both the compactness of $f$ and assumption $(\mathrm{H})$, we may construct a $\Gamma$-invariant, open neighborhood $N$ of $\partial \Omega$ such that $\bar{N} \subset B$ and

$$
\widehat{\Phi}(z) \neq 0 \text { for any } z \in \bar{N} \text {. }
$$

Set $V=B \backslash(\Omega \cup N)$. Clearly, $V$ is $\Gamma$-invariant, closed, and such that $V \cap \bar{\Omega}=$ $\varnothing$. Let $\varphi: B \rightarrow[0,1]$ be a $\Gamma$-invariant Urysohn function such that $\varphi(z)=0$ for $z \in \bar{\Omega}$ and $\varphi(z)=1$ if $z \in V$.

Let $F:[0,1] \times B \rightarrow \mathbf{R}^{N+1} \times E$ be the map defined as

$$
F(t, z)=(2 t+2 \varphi(z)-1, \widehat{\Phi}(z)) \text { if }(t, z) \in[0,1] \times B .
$$

Notice that $F$ is continuous and $\Gamma$-equivariant (the group $\Gamma$ acts trivially both on $[0,1]$ and on the first component of $\mathbf{R}^{N+1}$ ). Moreover, the map $F$ does not vanish on $\partial([0,1] \times B)$ and if $F(t, z)=0$ then $z \in \Omega, \widehat{\Phi}(z)=\Phi(z)=0$, and $t=\frac{1}{2}$. Thus, $F$ maps $\partial([0,1] \times B)$ into $\mathbf{R}^{N+1} \times E \backslash\{0\}$ and therefore we may consider the homotopy class $[F]_{\Gamma}$ of $F$. In analogy with the finite-dimensional case, we may consider the group of all $\Gamma$-equivariant homotopy classes induced by maps of the form (3.1). These groups will be denoted by $\Pi_{M, N}^{\Gamma}$. In this case the admissible $\Gamma$-homotopies are those of the form

$$
H(t, x, y ; \tau)=\left(f_{0}(t, x, y ; \tau),-\hat{f}_{N}(t, x, y ; \tau), y-\hat{f}_{\infty}(t, x, y ; \tau)\right)
$$

where $f_{0}$ is real valued, $\hat{f}_{N}$ has values in $\mathbf{R}^{N}$, and $\hat{f}_{\infty}$ is compact. Hence to the couple $(\Phi, \Omega)$ there corresponds an element $[F]_{\Gamma} \in \Pi_{M, N}^{\Gamma}$. It is easily verified that $[F]_{\Gamma}$ is independent of its construction and we may call it the generalized $\Gamma$-degree of $\Phi$ on $\Omega$, denoted by $\operatorname{deg}_{\Gamma}(\Phi, \Omega)$.

Clearly, $\operatorname{deg}_{\Gamma}(\Phi, \Omega)$ has the properties listed for the finite-dimensional case: existence, $\Gamma$-homotopy invariance, excision, and suspension. In fact, all of the corresponding proofs run as for the finite-dimensional case, with only minor modifications, taking into account the compactness of the $\Gamma$-equivariant map $f$ (see Remark 2.7). 
We proceed now to the task of computing $\operatorname{deg}_{\Gamma}(f, \Omega)$ by appealing to suitable finite-dimensional approximations of the couple $(\Phi, \Omega)$. As a by-product of this procedure we get a different way of defining $\Pi_{M, N}^{\Gamma}$. Assume now that the above assumptions hold and let us consider the $\Gamma$-extension $\hat{f}$ of $f$ to $B$ introduced in the construction of $\operatorname{deg}_{\Gamma}(\Phi, \Omega)$. As $\hat{f}$ is compact there exist both a sequence of finite-dimensional subspaces $E_{n}^{\prime}, n \in \mathbf{N}$, of $\mathbf{R}^{N} \times E$ and a sequence of continuous maps $f_{n}^{\prime}: B \rightarrow E_{n}^{\prime}, n \in \mathbf{N}$, such that

$$
\hat{f}(z)=\sum_{0}^{\infty} f_{n}^{\prime}(z) \text { and }\left|f_{n}^{\prime}(z)\right| \leq 2^{-n} \text { for all } z \in B, n \in \mathbf{N} .
$$

We may assume that $\mathbf{R}^{N} \subset E_{n}^{\prime}, n \in \mathbf{N}$. By averaging $f_{n}^{\prime}$ on the group we obtain a new sequence of maps $f_{n}$ (i.e., $f_{n}(z)=\int_{\Gamma} \gamma^{-1} f_{n}^{\prime}(\gamma z) d \gamma$ ) with the following properties (see [I, p. 783]).

(1) $f_{n}: B \rightarrow \Gamma E_{n}^{\prime}$ is $\Gamma$-equivariant,

(2) $\Gamma E_{n}^{\prime}=\bigcup_{\gamma \in \Gamma} \gamma E_{n}^{\prime}$ is a finite-dimensional $\Gamma$-invariant space,

(3) $\left\|f_{n}(z)\right\| \leq 2^{-n}$ for all $z \in B$ and $n \in \mathbf{N}$,

(4) $\hat{f}(z)=\sum_{n=0}^{\infty} f_{n}(z)$ for all $z \in B$.

Now let $\widetilde{E}_{n}=\left\langle\mathbf{R}^{N}, \Gamma E_{1}^{\prime}, \Gamma E_{2}^{\prime}, \ldots, \Gamma E_{n}^{\prime}\right\rangle=\mathbf{R}^{N} \oplus E_{n}, n \in \mathbf{N}$. Clearly, $\widetilde{E}_{n}$ is a $\Gamma$-invariant finite-dimensional space. Consider the sequence of maps $\hat{f}_{n}(z)=$ $\sum_{i=0}^{n} f_{i}(z), n \in \mathbf{N}$. Each map $\hat{f}_{n}: B \rightarrow \widetilde{E}_{n}, n \in \mathbf{N}$, is $\Gamma$-equivariant and such that

$$
\left\|\hat{f}(z)-\hat{f}_{n}(z)\right\| \leq 2^{-n} \text { for all } z \in B .
$$

Let $P_{n}^{\prime}$ be a projection of $E$ onto $E_{n}$ and let $P_{n}$ be its average over the group $\Gamma$ (thus $P_{n}$ is $\Gamma$-equivariant; see [V, p. 94]).

Finally, set $P_{n} y=y_{n}, y \in E$. Now, since $\hat{f}$ is compact and $\|\widehat{\Phi}(z)\| \geq 2 \varepsilon>$ 0 for all $z \in \bar{N}$, where $N$ is the $\Gamma$-invariant open neighborhood of $\partial \Omega$ used in the definition of the $\Gamma$-degree of $f$, it follows that there exists $n_{0} \in \mathbf{N}$ such that for $n \geq n_{0}$

$$
\left\|y-\hat{f}_{n}(x, y)\right\| \geq \varepsilon \text { for all }(x, y) \in \bar{N} .
$$

For any $n \in \mathbf{N}$, set $B^{n}=B \cap\left(\mathbf{R}^{M} \times E_{n}\right)$ and let $\widehat{\Phi}_{n}: B^{n} \rightarrow \mathbf{R}^{N} \times E_{n}$ be the $\Gamma$-equivariant map defined as

$$
\widehat{\Phi}_{n}\left(x_{n}, y_{n}\right)=y_{n}-\hat{f}_{n}\left(x_{n}, y_{n}\right), \text { where } y_{n}=P_{n} y .
$$

Let $\Omega_{n}=\Omega \cap\left(\mathbf{R}^{M} \times E_{n}\right)$ and $\bar{N}_{n}=\bar{N} \cap\left(\mathbf{R}^{M} \times E_{n}\right)$. Since $\partial \Omega_{n} \subset \bar{N}_{n} \cap\left(\mathbf{R}^{M} \times E_{n}\right)$, we have that the $\Gamma$-equivariant map $\Phi_{n}: \bar{\Omega}_{n} \rightarrow \widetilde{E}_{n}$ defined as the restriction of $\widehat{\Phi}_{n}$ to $\bar{\Omega}_{n}$ does not vanish on $\partial \Omega_{n}$ by (3.3), provided $n \geq n_{0}$. Therefore $\operatorname{deg}_{\Gamma}\left(\Phi_{n}, \Omega_{n}\right)$ is well defined. Furthermore

$$
\operatorname{deg}_{\Gamma}(\Phi, \Omega)=\Sigma^{\widetilde{E}} \operatorname{deg}_{\Gamma}\left(\Phi_{n}, \Omega_{n}\right)
$$


where $\Sigma^{\widetilde{E}}$ stands for the $\widetilde{E}$ - $\Gamma$-equivariant suspension and $\widetilde{E}$ is a $\Gamma$-invariant subspace of $E$ such that $E=E_{n} \oplus \widetilde{E}$. Indeed, by (3.2) and (3.3) the map $\Phi$ is $\Gamma$-homotopic to the map $\widetilde{\Phi}(x, y)=y-f_{n}(x, y)$. Decompose $y=$ $\left(y_{n}, \tilde{y}\right)$, where $y_{n} \in E_{n}$ and $\tilde{y} \in \tilde{E}$. Then the map $\widetilde{\Phi}$ is $\Gamma$-homotopic to $\left(y_{n}-f_{n}\left(x, y_{n}, 0\right), \tilde{y}\right)$, which can be thought as the $\widetilde{E}$ - $\Gamma$-suspension of $\Phi_{n}$. This brings immediately to one's mind the idea of relating the $\Gamma$-degree of the original map $\Phi$ with the $\Gamma$-degree of its finite-dimensional approximations $\Phi_{n}$. This is, of course, nothing else but an adaptation of the classical technique due to Leray and Schauder when constructing the topological degree for compact perturbations of the identity via the Brouwer degree of their finite-dimensional approximations.

To be more precise, we have to proceed by comparing $\operatorname{deg}_{\Gamma}\left(\Phi_{n}, \Omega_{n}\right)$ and $\operatorname{deg}_{\Gamma}\left(\Phi_{m}, \Omega_{m}\right)$ for $n, m \geq n_{0}$. To this end, some further notation and preliminary results are needed. Denote with $E_{n, m}$ the $\Gamma$-invariant space $E_{n, m}=$ $\left\langle E_{n}, E_{m}\right\rangle$ and let $P_{n, m}$ be a $\Gamma$-equivariant projection onto $E_{n, m}$. Set

$$
\widetilde{E}=\left(I-P_{n}\right) \circ P_{n, m}\left(\mathbf{R}^{N} \times E\right)
$$

and let $\tilde{y}=\left(I-P_{n}\right) \circ P_{n, m} y$. Clearly the space $\widetilde{E}$ is $\Gamma$-invariant and $E_{n} \oplus \widetilde{E}=$ $E_{n, m}$. Put $\Omega_{n, m}=\Omega \cap\left(\mathbf{R}^{N} \times E_{n, m}\right)$, let

$$
\widehat{\Psi}: \bar{B} \cap\left(\mathbf{R}^{M} \times E_{n, m}\right) \rightarrow \mathbf{R}^{N} \times E_{n, m}
$$

be the $\Gamma$-equivariant map defined by $\hat{\Psi}\left(x, y_{n}, \tilde{y}\right)=\left(y_{n}-\hat{f}_{n}\left(x, y_{n}, \tilde{y}\right), \tilde{y}\right)$ and set $\Psi=\widehat{\Psi}_{\mid \bar{\Omega}_{n, m}}$. Notice that $\Psi_{\mid \mathbf{R}^{M} \times E_{n}}=\Phi_{n}$. We are now in a position of proving the following.

Proposition 3.1. $\operatorname{deg}_{\Gamma}\left(\Psi, \Omega_{n, m}\right)=\Sigma^{\widetilde{E}} \operatorname{deg}_{\Gamma}\left(\Phi_{n}, \Omega_{n}\right)$, where $\operatorname{deg}_{\Gamma}\left(\Psi, \Omega_{n, m}\right) \in$ $\pi_{M+n+\operatorname{dim} \widetilde{E}}^{\Gamma}\left(S^{N+n+\operatorname{dim} \widetilde{E}}\right)$ and $\Sigma^{\widetilde{E}} \operatorname{deg}_{\Gamma}\left(\Phi_{n}, \Omega_{n}\right)$ is the $\widetilde{E}$ - $\Gamma$-equivariant suspension of the element $\operatorname{deg}_{\Gamma}\left(\Phi_{n}, \Omega_{n}\right) \in \pi_{M+n}^{\Gamma}\left(S^{N+n}\right)$.

Proof. Note first that by the excision property of the $\Gamma$-degree we may replace the set $\Omega_{n, m}$ by the set $\Omega_{n, m} \cup\left(\Omega_{n} \times\{\tilde{y} \in \tilde{E}:\|\tilde{y}\|<\varepsilon\}\right)$ and this, in its turn, by the set $\Omega_{n} \times\{\tilde{y} \in \tilde{E}:\|\tilde{y}\|<\varepsilon\}$. We may also deform the map $\widetilde{\Psi}$ to the map

$$
\tilde{\Psi}^{\prime}\left(x, y_{n}, \tilde{y}\right)=\left(y_{n}-\hat{f}_{n}\left(x, y_{n}, 0\right), \tilde{y}\right) .
$$

Set $N_{n}=N \cap\left(\mathbf{R}^{M} \times E_{n}\right)$ and $\varphi_{n}=\varphi_{\mid B^{n}}$, where $\varphi$ is the Urysohn function associated to $N$. Obviously, $\partial \Omega_{n} \subset N_{n}$ and $\varphi_{n}$ is a $\Gamma$-invariant Urysohn function associated to $N_{n}$.

If we set $B_{r}^{\prime}=\{\tilde{y} \in \widetilde{E}:\|\tilde{y}\|<r\}$, then $\bar{N}_{n} \times B_{2 \varepsilon}^{\prime}$ is a $\Gamma$-invariant neighborhood of $\partial\left(\Omega_{n} \times B_{\varepsilon}^{\prime}\right)$ such that

$$
\partial\left(\bar{N}_{n} \times B_{2 \varepsilon}^{\prime}\right) \cap\left(\Omega_{n} \times B_{\varepsilon}^{\prime}\right)^{c}=\varnothing
$$


and, by (3.3), $\widehat{\Psi} \neq 0$ on $\bar{N}_{n} \times B_{2 \varepsilon}^{\prime}$. Let $\psi: \bar{B} \cap\left(\mathbf{R}^{M} \times E_{n, m}\right) \rightarrow[0,1]$ be defined as

$$
\psi\left(x, y_{n}, \tilde{y}\right)=\left\{\begin{array}{l}
\varphi_{n}\left(x, y_{n}\right) \quad \text { if }\|\tilde{y}\| \leq \varepsilon, \\
\varphi_{n}\left(x, y_{n}\right)(2-\|\tilde{y}\| / \varepsilon)-1+\|\tilde{y}\| / \varepsilon \quad \text { if } \varepsilon \leq\|\tilde{y}\| \leq 2 \varepsilon, \\
1 \text { if } 2 \varepsilon \leq\|\tilde{y}\| .
\end{array}\right.
$$

Clearly $\psi$ is a $\Gamma$-invariant Urysohn function associated to $\bar{N}_{n} \times B_{2 \varepsilon}^{\prime}$. It follows that in the $\Gamma$-homotopy class of $\operatorname{deg}_{\Gamma}\left(\psi, \Omega_{n, m}\right)$ we have the map

$$
\left(2 t+2 \psi(z)-1, \hat{\Psi}^{\prime}(z)\right), \quad t \in[0,1], z \in B \cap\left(\mathbf{R}^{M} \times E_{n, m}\right),
$$

which can be deformed (via a convex $\Gamma$-homotopy) to the map

$$
\left(2 t+2 \varphi_{n}\left(x, y_{n}\right)-1, y_{n}-\hat{f}_{n}\left(x_{n}, y_{n}\right), \tilde{y}\right) .
$$

Note that if $\tilde{y}=0$ then the first components of the maps (3.4) and (3.5) coincide. The map (3.5) is clearly the $\widetilde{E}-\Gamma$-suspension of the map associated to

$$
\Phi_{n}: \Omega_{n} \rightarrow \mathbf{R}^{N} \times E_{n}
$$

defined through (3.1). Q.E.D.

Clearly Proposition 3.1 can be applied equally well to the maps $\Phi_{m}$ and $\boldsymbol{\Theta}=\widehat{\boldsymbol{\Theta}}_{\left.\right|_{\Omega_{n, m}}}$ where the map $\widehat{\boldsymbol{\Theta}}: B \cap\left(\mathbf{R}^{M} \times E_{n, m}\right) \rightarrow \mathbf{R}^{N} \times E_{n, m}$ is defined by $\widehat{\Theta}\left(x, y_{m}, \hat{y}\right)=\left(y_{m}-\hat{f}_{m}\left(x, y_{m}, \hat{y}\right), \hat{y}\right)$ with $\hat{y}_{m}=P_{m} y, P_{m}$ a $\Gamma$-equivariant projection onto $E_{m}$ and $\tilde{y} \in \widehat{E}=\left(I-P_{m}\right) \circ P_{n, m} E$. Hence, we have that

$$
\operatorname{deg}_{\Gamma}\left(\boldsymbol{\Theta}, \Omega_{n, m}\right)=\Sigma^{\widehat{E}} \operatorname{deg}_{\Gamma}\left(\Phi_{m}, \Omega_{m}\right)
$$

Finally, using (3.2) and (3.3), it follows that the maps $\widehat{\Phi}$ and $\widehat{\Theta}$ are $\Gamma$ homotopic on $B \cap\left(\mathbf{R}^{N} \times E_{n, m}\right)$ (take a convex $\Gamma$-homotopy). Therefore

$$
\Sigma^{\widetilde{E}} \operatorname{deg}_{\Gamma}\left(\Phi_{n}, \Omega_{n}\right)=\Sigma^{\widehat{E}} \operatorname{deg}_{\Gamma}\left(\Phi_{m}, \Omega_{m}\right) \text { provided } n, m>n_{0} .
$$

To perform the last step of our construction we assume that the $\Gamma$-equivariant Freudenthal suspension theorem applies (see Theorem B.1 in the Appendix).

We may now define $\operatorname{deg}_{\Gamma}(\Phi, \Omega)$ as the direct limit of the finite-dimensional $\Gamma$-degrees $\operatorname{deg}_{\Gamma}\left(\Phi_{n}, \Omega_{n}\right)$ and $\Pi_{M, N}^{\Gamma}=\lim _{\rightarrow} \pi_{M+\operatorname{dim} E_{n}}^{\Gamma}\left(S^{N+\operatorname{dim} E_{n}}\right)$. This is an alternative approach to defining the $\Gamma$-degree in infinite dimension.

Remark 3.2.

(i) The $\Gamma$-degree for mappings between infinite-dimensional spaces has the complete additivity property in the case when the suspension is an isomorphism (see Appendix B).

(ii) If $\Gamma=\{e\}$ and $M=N$, then $\operatorname{deg}_{\Gamma}(\Phi, \Omega)=\operatorname{deg}_{L S}(\Phi, \Omega)$, where $\operatorname{deg}_{L S}$ denotes the Leray-Schauder degree for the compact vector field $\Phi$ (see Property (g) in $\S 2$ ). 
(iii) When defining the degree through finite-dimensional approximations, one has to keep track of the suspension isomorphisms used, in particular, of the orientation chosen.

(iv) The reader can see easily that one may extend this degree to other categories of maps: $\Gamma-k$-set contractions, $\Gamma$ - $A$-proper, $\Gamma-C^{1}$-Fredholm nonlinear operators, and other classes as considered in [I.M.P.V].

We close this section with some results related to global continuation and global bifurcation problems.

It is clear that once the degree is defined one may use it to get the global results about existence and dimension which were given in [I.M.P.V] and [I.M.V] since the notion of $\Gamma$-epi map is more general than that of the $\Gamma$-degree (see property (f) in $\S 2)$.

For example, for continuation problems, suppose one considers the equation $x-F(x, \lambda)=0$ in an open bounded $\Gamma$-invariant subset $\Omega$ of $\mathbf{R}^{M} \times E \times \Lambda$, where $F$ is a compact, $\Gamma$-equivariant map with values in $\mathbf{R}^{N} \times E$. The group $\Gamma$ may also act on $\Lambda$. Assume that for some $\lambda_{0} \in \Lambda^{\Gamma}, x-F\left(x, \lambda_{0}\right) \neq 0$ on $\partial \Omega_{\lambda_{0}}=\partial\left\{(x, \lambda) \in \Omega: \lambda=\lambda_{0}\right\}$ and $\operatorname{deg}_{\Gamma}\left(x-F\left(x, \lambda_{0}\right), \Omega_{\lambda_{0}}, 0\right) \neq 0$. Assume further that the $\Gamma$-equivariant Freudenthal suspension theorem applies and that $\Lambda=\Lambda^{\Gamma} \oplus \Lambda_{1}$ with $\operatorname{dim} \Lambda^{\Gamma}>0$.

Then there exists a "continuum" $\Sigma$ of solutions of the equation $x-F(x, \lambda)=$ 0 , with $\lambda \in \Lambda^{\Gamma}$, such that $\bar{\Sigma} \cap \partial \Omega \neq \varnothing$ and $\Sigma / \Gamma$ is connected and has dimension at each point at least $\operatorname{dim} \Lambda^{\Gamma}$.

Indeed, consider the map $H(x, \lambda)=\left(x-F(x, \lambda), \lambda-\lambda_{0}\right)$. Clearly, $H$ is $\Gamma$ equivariant, nonzero on $\partial \Omega$, and $\operatorname{deg}_{\Gamma}(H, \Omega)=\Sigma^{\Lambda} \operatorname{deg}_{\Gamma}\left(x-F\left(x, \lambda_{0}\right), \Omega_{\lambda_{0}}, 0\right)$. Thus, the map $H$ is $\Gamma$-epi on $\Omega$ and then the map $\lambda-\lambda_{0}$ is $\Gamma$-epi on the set of zeros of $x-F(x, \lambda)$, with $\lambda \in \Lambda^{\Gamma}$. Now one may apply Theorem 3.1 of [I.M.V].

For bifurcation problems one considers, as before, an equation of the form $x-F(x, \lambda)=0$ with $F(0, \lambda)=0$ for any $\lambda \in \Lambda$. Assume that $F$ is $C^{1}$ and that for some $\lambda_{0} \in \Lambda^{\Gamma}$ the map $I-F_{x}\left(0, \lambda_{0}\right)$ is noninvertible but $I-F_{x}(0, \lambda)$ is invertible for $0<\left|\lambda-\lambda_{0}\right| \leq \rho$ with $\lambda \in \tilde{\Lambda}$ (where $\tilde{\Lambda}$ is some invariant subspace of $\Lambda$ ). Let $\widehat{\Lambda}$ be an invariant subspace of $\Lambda$ such that $\Lambda=\widetilde{\Lambda} \oplus \widehat{\Lambda}$ and let $\widetilde{B}_{\rho}=\left\{\tilde{\lambda}:\left|\tilde{\lambda}-\tilde{\lambda}_{0}\right|<\rho\right\}$. Finally, assume that

$$
\operatorname{deg}_{\Gamma}\left(\left(x-F_{x}(0, \tilde{\lambda}, 0) x,\|x\|-\varepsilon\right), B_{2 \varepsilon} \times \widetilde{B}_{\rho}\right) \neq 0
$$

and one is in a position to apply the $\Gamma$-equivariant Freudenthal suspension theorem. (If $\operatorname{dim} \tilde{\Lambda}=1$, then the above inequality holds if and only if

$$
\left.\operatorname{deg}_{\Gamma}\left(x-F_{x}(0, \tilde{\lambda}-\rho, 0) x, B_{2 \varepsilon}\right) \neq \operatorname{deg}_{\Gamma}\left(x-F_{x}(0, \tilde{\lambda}+\rho, 0) x, B_{2 \varepsilon}\right)\right) .
$$

Then there exists a branch $\Sigma$ of solutions bifurcating from $\left(0, \lambda_{0}\right)$ with $\hat{\lambda}=0$ such that

(1) $\Sigma$ is either unbounded or returns to $(0, \tilde{\lambda}, 0)$. 
(2) The local dimension of $\Sigma / \Gamma$ is at least $\operatorname{dim} \widehat{\Lambda}^{\Gamma}+1$.

(3) If the return points on $\Sigma$ have a linearization, with the same invertibility properties assumed for $\lambda_{0}$, and $\Sigma$ is bounded, then the sum of the local $\Gamma$-indices, $\operatorname{deg}_{\Gamma}\left(\left(x-F\left(0, \tilde{\lambda}_{i}, 0\right) x,\|x\|-\varepsilon\right), B_{2 \varepsilon} \times B_{\rho}\right)$, is trivial.

(4) If for some subgroup $H$ of $\Gamma$ one knows that if $x^{H}-F^{H}\left(x^{H}, \tilde{\lambda}^{H}, 0\right)$ $=0$ for $\left|\lambda^{H}-\lambda_{0}^{H}\right| \leq \rho$ implies $x^{H}=0$-that is, there is no bifurcation in $\left(\mathbf{R}^{M} \times E \times \tilde{\Lambda}\right)^{H}$-then (1), (2), and (3) hold for a subset $\Sigma$ in the complement of $E^{H}$. The return points, if there are any, belong to $E^{H}$. In particular, for $H=\Gamma$, one has nonstationary solutions.

Indeed, the map $\left(x-F(x, \lambda),\|x\|-\varepsilon, \hat{\lambda}-\hat{\lambda}_{0}\right)$ has $\Gamma$-degree on $B_{2 \varepsilon} \times B_{\rho}$, for $\varepsilon$ small enough, equal to $\Sigma^{\hat{\Lambda}} \operatorname{deg}_{\Gamma}\left(x-F_{x}(0, \tilde{\lambda}, 0),\|x\|-\varepsilon\right)$. Then the argument proceeds as in [I.M.P.V] and [I.M.V, Theorems 3.1 and 4.2]. Note that assertion (3) is obtained by using the arguments of $\left[\mathrm{I}_{0}\right.$, p. 77] (there is no need for cohomotopy here). To obtain (4), one has to complement the equation $x-$ $F(x, \lambda)=0$ with $\left\|x_{1}\right\|-\varepsilon$ instead of $\|x\|-\varepsilon$, where $x_{1}$ is in the complement of $E^{H}$. Finally note that here $\operatorname{dim}(\mathbf{R} \times \Lambda)^{\Gamma} \geq 1$.

One may also refine these results using the different ideas and theorems proved in [I.M.V].

\section{THE $S^{1}$-EQUIVARIANT DEgREE}

In this part of the paper we shall restrict our attention to the case $\Gamma=S^{1}$. We will give a complete characterization of the $S^{1}$-degree when a semifree (actually almost semifree) action is considered. More precisely, if $S^{1}$ acts on $\mathbf{R}^{k} \times \mathbf{C}^{m}$ and $\mathbf{R}^{l} \times \mathbf{C}^{n}$, leaving fixed $\mathbf{R}^{k}$ and $\mathbf{R}^{l}$ respectively, and multiplying each component of $\mathbf{C}^{m}$ by $e^{i m_{0} \varphi}$, where $m_{0} \geq 1$, and each component of $\mathbf{C}^{n}$ by $e^{i n_{j} \varphi}$, where $n_{j}$ are multiples of $m_{0}$, then we shall give in Corollaries 4.1 and 4.2 a complete description of $\pi_{k+2 m}^{S^{1}}\left(S^{l+2 n}\right)$ whenever $m=1$ or $m \leq$ $n+1-(k-l) / 2$ (any of these numbers may be zero). When the action of $S^{1}$ on $\mathrm{C}^{m}$ is not of one orbit type (see [B, p. 42]), we shall construct an auxiliary degree which has all the properties of a degree by reducing the problem to an almost semifree action. This auxiliary degree can also be extended to infinite dimensions. In particular, if $k=l+1$ and $m=n$ (these last numbers may be infinite), we extend the $S^{1}$-degree introduced in [I.M.V] to general domains, recovering both Fuller's and Dancer's degrees (see [F] and [Da]).

We emphasize that we obtain this extension without referring to the formalism of obstruction theory.

We shall explore further the $S^{1}$-degree (when the action is not necessarily almost semifree) in the second paper of this series.

4.1. The finite-dimensional case, reduction to semifree action. Let $\mathbf{R}^{M}, \mathbf{R}^{N}$ be linear representations of $S^{1}$ with fixed-point subspaces $\mathbf{R}^{k}$ and $\mathbf{R}^{l}$ respectively. 
Then the action of $S^{1}$ on the orthogonal complements of those subspaces will give them a complex structure. Thus $\mathbf{R}^{M}=\mathbf{R}^{k} \times \mathbf{C}^{m}, \mathbf{R}^{N}=\mathbf{R}^{l} \times \mathbf{C}^{n}$, and the action will be represented by

$$
\begin{aligned}
\mathbf{e}^{i m \varphi} z & :=\mathbf{e}^{i m \varphi}\left(z_{1}, \ldots, z_{m}\right)=\left(e^{i m_{1} \varphi} z_{1}, \ldots, e^{i m_{m} \varphi} z_{m}\right), \\
\mathbf{e}^{i n \varphi} \xi & :=\mathbf{e}^{i n \varphi}\left(\xi_{1}, \ldots, \xi_{n}\right)=\left(e^{i n_{1} \varphi} \xi_{1}, \ldots, e^{i n_{n} \varphi} \xi_{n}\right),
\end{aligned}
$$

where $z_{i}, \xi_{j}$ are complex numbers and $m_{i}, n_{j}$ are nonzero integers, $i=$ $1, \ldots, m, j=1, \ldots, n$. By taking conjugates if necessary, we may assume without loss of generality that $m_{i}$ and $n_{j}$ are positive and form a nondecreasing sequence of integers (conjugation changes only the orientation in $\mathbf{C}^{m}$ and $\mathbf{C}^{n}$ and induces isomorphisms at the level of the equivariant homotopy groups). We set $M^{\prime}=\prod_{i=1}^{m} m_{i}, N^{\prime}=\prod_{i=1}^{n} n_{i}$, and let $m_{0}$ be a common divisor of $m_{i}$, $i=1, \ldots, m$.

Any element of $\mathbf{R}^{M}$ will be written as $\left(x_{0}, z\right) \in \mathbf{R}^{k} \times \mathbf{C}^{m}$ and any $S^{1}$. equivariant map $F$ from an invariant subset of $\mathbf{R}^{M}=\mathbf{R}^{k} \times \mathbf{C}^{m}$ into $\mathbf{R}^{N}=\mathbf{R}^{l} \times$ $\mathbf{C}^{n}$ will have the form $\left(\Phi_{0}\left(x_{0}, z\right), \Phi\left(x_{0}, z\right)\right)$ where $\Phi_{0}\left(x_{0}, \mathbf{e}^{i m \varphi} z\right)=\Phi_{0}\left(x_{0}, z\right)$ and $\Phi\left(x_{0}, \mathbf{e}^{i m \varphi} z\right)=\mathbf{e}^{i n \varphi} \Phi\left(x_{0}, z\right)$. Note that due to the equivariance of $F$ the integer $m_{0}$ divides $n_{j}, j=1, \ldots, n$, provided that the $j$ th component of $\Phi$ is not identically equal to zero. Finally, observe that the radial scaling from the ball $\left\{\left(x_{0}, z\right):\left\|x_{0}\right\|^{2}+\|z\|^{2} \leq R_{0}^{2}+R^{2}\right\}$ to the set

$$
D=\left\{\left(x_{0}, z\right):\left\|x_{0}\right\| \leq R_{0},\|z\| \leq R\right\}
$$

is a $S^{1}$-equivariant homeomorphism which induces an isomorphism at the equivariant homotopy level. We shall then identify the $S^{1}$-homotopy group of equivariant maps from $\partial([0,1] \times D)$ into $\mathbf{R} \times \mathbf{R}^{N} \backslash\{0\}$ of the form $\left(f_{0}(x, z)\right.$, $\left.\Phi_{0}(x, z), \Phi(x, z)\right)$ with $\pi_{k+2 m}^{S^{1}}\left(S^{l+2 n}\right)$. Here the variable $x$ stands for the pair $\left(t, x_{0}\right) \in[0,1] \times \mathbf{R}^{k}$. In the case of the generalized $\Gamma$-degree, the map $f_{0}$ is the auxiliary real-valued function $f_{0}\left(x_{0}, z\right)=2 t+2 \varphi\left(x_{0}, z\right)-1$, and the pair of maps $\left(\Phi_{0}, \Phi\right)$ does not depend explicitly on the variable $t$.

Let $\mathbf{C}^{m}$. denote $\mathbf{C}^{m}$ with the following $S^{1}$-action:

$$
\mathrm{e}^{i m_{0} \varphi} Z:=\mathrm{e}^{i m_{0} \varphi}\left(Z_{1}, \ldots, Z_{m}\right)=\left(e^{i m_{0} \varphi} Z_{1}, \ldots, e^{i m_{0} \varphi} Z_{m}\right),
$$

which reduces to the standard free action of $S^{1}$ when $m_{0}=1$.

Then, leaving $S^{1}$ to act trivially on $\mathbf{R}^{k}$, we shall refer to the action on $\mathbf{R}^{k} \times \mathbf{C}^{m}$ as an almost semifree $S^{1}$-action. Now, let $\boldsymbol{\Theta :} \mathbf{R}^{k} \times \mathbf{C}^{m} \rightarrow \mathbf{R}^{k} \times \mathbf{C}^{m}$ be the $S^{1}$-equivariant map defined by

$$
\Theta\left(x_{0}, Z_{1}, \ldots, Z_{m}\right)=\left(x_{0}, Z_{1}^{\mu_{1}}, \ldots, Z_{m}^{\mu_{m}}\right), \quad \text { where } m_{0} \mu_{i}=m_{i},
$$

and let $\pi_{k+2 m}^{S^{1}},\left(S^{l+2 n}\right)$ be the group of all equivariant homotopy classes of $S^{1}$-maps from the boundary of the set $D^{\prime}=\left\{\left(t, x_{0}, Z\right): t \in[0,1],\left\|x_{0}\right\| \leq\right.$ 
$\left.R_{0},\|Z\| \leq R\right\}$ into $\mathbf{R} \times \mathbf{R}^{l} \times \mathbf{C}^{n} \backslash\{0\} \quad$ (define the addition as for the group $\left.\pi_{k+2 m}^{S^{1}}\left(S^{l+2 n}\right)\right)$.

The following results are in order.

Lemma 4.1. The map $\Theta$ induces a group homomorphism

$$
\Theta^{*}: \pi_{k+2 m}^{S^{1}}\left(S^{l+2 n}\right) \rightarrow \pi_{k+2 m}^{S^{1}},\left(S^{l+2 n}\right) .
$$

Proof. Let $F\left(t, x_{0}, z\right)=\left(f_{0}, \Phi_{0}, \Phi\right)\left(t, x_{0}, z\right)$ be a $S^{1}$-equivariant map from $[0,1] \times D$ into $\mathbf{R} \times \mathbf{R}^{l} \times \mathbf{C}^{n}$ such that $F(\partial([0,1] \times D)) \subset \mathbf{R} \times \mathbf{R}^{l} \times \mathbf{C}^{n} \backslash\{0\}$ where $D$ is as in (4.2). Consider the set

$$
\widetilde{D}=\left\{\left(t, x_{0}, Z\right): t \in[0,1], \Theta\left(x_{0}, Z\right) \in D\right\} .
$$

Clearly, $\widetilde{D}$ is a $S^{1}$-invariant set with respect to the $S^{1}$-almost semifree action and the map

$$
\widetilde{F}\left(t, x_{0}, Z\right)=F\left(t, \Theta\left(x_{0}, Z\right)\right)
$$

from $\widetilde{D}$ into $\mathbf{R} \times \mathbf{R}^{l} \times \mathbf{C}^{n}$ is $S^{1}$-equivariant (note that $\widetilde{F}(\partial \widetilde{D}) \subset \mathbf{R} \times \mathbf{R}^{l} \times \mathbf{C}^{n} \backslash\{0\}$ ). Moreover, the sets $D$ and $\widetilde{D}$ being homeomorphic via a radial scaling (hence $S^{1}$-invariant), we may identify the set of all equivariant homotopy classes of $S^{1}$-maps defined on the set $\widetilde{D}$ with $\pi_{k+2 m}^{S^{1}},\left(S^{l+2 n}\right)$. Let $\Theta^{*}\left([F]_{S^{1}}\right)=[\widetilde{F}]_{S^{1}}$, where $\widetilde{F}$ is given by (4.3). Clearly, the morphism $\Theta^{*}$ does not depend on the choice of the map $F$ but only on its homotopy class. Hence the morphism $\Theta^{*}$ is well defined. Finally, $\Theta^{*}$ is a group homomorphism since the addition is an operation on the $t$ variable only (where the group $S^{1}$ acts trivially). Q.E.D.

Lemma 4.2. Let $\Omega$ be an open and bounded $S^{1}$-invariant subset of $\mathbf{R}^{k} \times \mathbf{C}^{m}$ and let $f: \partial \Omega \rightarrow \mathbf{R}^{l} \times \mathbf{C}^{n} \backslash\{0\}$ be $S^{1}$-equivariant (the action is the one described by (4.1)). Then

$$
\Theta^{*} \operatorname{deg}_{S^{1}}(f, \Omega)=\operatorname{deg}_{S^{1}}\left(f \circ \Theta, \Theta^{-1}(\Omega)\right) .
$$

Proof. Let $R_{0}, R$ be such that $\Omega \subset D=\left\{\left(x_{0}, z\right) \in \mathbf{R}^{k} \times \mathbf{C}^{m}:\left\|x_{0}\right\| \leq R_{0}\right.$, $\|z\| \leq R\}$. As in $\S 2$, let $\hat{f}: D \rightarrow \mathbf{R}^{l} \times \mathbf{C}^{n}$ be a $S^{1}$-equivariant extension of $f$ to $D$, let $N$ be a $S^{1}$-invariant neighborhood of $\partial \Omega$ on which $\hat{f} \neq 0$, and let $\varphi$ be a $S^{1}$-invariant Urysohn function associated to $N$. Thus $\operatorname{deg}_{S^{1}}(f, \Omega)=[F]_{S^{1}}$, where $F\left(t, x_{0}, z\right)=\left(2 t+2 \varphi\left(x_{0}, z\right)-1, \hat{f}\left(x_{0}, z\right)\right)$. Now consider $U=\boldsymbol{\Theta}^{-1}(\Omega)$. Then $U$ is an open bounded and $S^{1}$-invariant subset of $\mathbf{R}^{k} \times \mathbf{C}^{m}$, the set $\Theta^{-1}(N)$ is a $S^{1}$-invariant neighborhood of $\partial U$, and $\tilde{\varphi}=\varphi \circ \Theta$ is a $S^{1}$ invariant Urysohn function over $\Theta^{-1}(N)$ taking the right values on the different $S^{1}$-invariant sets. Hence, $\operatorname{deg}_{S^{1}}(f \circ \Theta, U)$ can be defined in terms of the map $\widetilde{F}\left(t, x_{0}, Z\right)=F\left(t, \Theta\left(x_{0}, Z\right)\right)$ and $\operatorname{deg}_{S^{1}}(f \circ \Theta, U)=[\widetilde{F}]_{S^{1}}$ (see $\left.\S 2\right)$. It is now a direct consequence of Lemma 4.1 that

$$
\Theta^{*} \operatorname{deg}_{S^{1}}(f, \Omega)=\operatorname{deg}_{S^{1}}\left(f \circ \Theta, \Theta^{-1}(\Omega)\right) \text {. Q.E.D. }
$$


On the basis of (4.4), we may consider from now on $S^{1}$-equivariant maps from $\mathbf{R}^{k} \times \mathbf{C}_{\text {. }}^{m}$ into $\mathbf{R}^{l} \times \mathbf{C}^{n}$.

We now introduce the following morphism of groups. Let $F\left(t, x_{0}, z\right)=$ $\left(f_{0}, \Phi_{0}, \Phi\right)\left(t, x_{0}, z\right)$ be a $S^{1}$-equivariant map from $\partial([0,1] \times D)$ into $\mathbf{R} \times$ $\mathbf{R}^{l} \times \mathbf{C}^{n} \backslash\{0\}$ where $D$ is the set defined by (4.2). Due to the $S^{1}$-action, we have that $\Phi\left(t, x_{0}, 0\right)=0$. Thus to the $S^{1}$-map $F$ we can associate the $S^{1}$ invariant map $\left(f_{0}, \Phi_{0}\right): \partial\left([0,1] \times\left\{x_{0} \in \mathbf{R}^{k}:\left\|x_{0}\right\| \leq R_{0}\right\}\right) \rightarrow \mathbf{R} \times \mathbf{R}^{l}$ defined as $\left(f_{0}, \Phi_{0}\right)\left(t, x_{0}\right)=\left(f_{0}, \Phi_{0}\right)\left(t, x_{0}, 0\right)$. Call this assignment $p$. Then we have the following.

Lemma 4.3. The map $p$ induces a group homomorphism

$$
p_{*}: \pi_{k+2 m, \cdot}^{S^{1}}\left(S^{l+2 n}\right) \rightarrow \pi_{k}\left(S^{l}\right)
$$

which is onto if $m \leq n$.

Furthermore, assuming $m \leq n$ and letting $F=\left(f_{0}, \Phi_{0}, \Phi\right)$ represent an element of $\pi_{k+2 m, \cdot}^{S^{1}}\left(S^{l+2 n}\right)$, the assignment

$$
[F]_{S^{1}} \mapsto\left(p_{*}\left([F]_{S^{1}}\right),[F]_{S^{1}}-[\tilde{F}]_{S^{1}}\right)
$$

from $\pi_{k+2 m, \cdot}^{S^{1}}\left(S^{l+2 n}\right)$ onto $\pi_{k}\left(S^{l}\right) \times \operatorname{ker} p_{*}$ is an isomorphism, where $\widetilde{F}=\left(f_{0}, \Phi_{0}\right.$, $\widetilde{\Phi})$ with

$$
\widetilde{\Phi}\left(t, x_{0}, Z\right)=t(1-t)(Z_{1}^{n_{1} / m_{0}}, \ldots, Z_{m}^{n_{m} / m_{0}}, \underbrace{0, \ldots, 0}_{n-m}) .
$$

(Recall that the map $F$ can be chosen such that $F\left(0, x_{0}, Z\right)=F\left(1, x_{0}, Z\right)=$ $(1,0,0)$; see Proposition A.1.)

Proof. The fact that $p_{*}$ is a morphism follows directly from the definition of the addition. Furthermore, if $\left[\left(f_{0}, \Phi_{0}\right)\right]$ is an element in $\pi_{k}\left(S^{l}\right)$ then the map

$$
F\left(t, x_{0}, Z\right)=(f_{0}\left(t, x_{0}\right), \Phi_{0}\left(t, x_{0}\right), Z_{1}^{n_{1} / m_{0}}, \ldots, Z^{n_{m} / m_{0}}, \underbrace{0, \ldots, 0}_{n-m})
$$

has the property that $p_{*}[F]_{S^{1}}=\left[\left(f_{0}, \Phi_{0}\right)\right]$; that is, $p_{*}$ is onto.

It remains to prove the last part of the assertion. To this end, recall that the element $[F]_{S^{1}}-[\widetilde{F}]_{S^{1}}$ is represented by

$$
G\left(t, x_{0}, Z\right)= \begin{cases}F\left(2 t, x_{0}, Z\right) & \text { if } 0 \leq t \leq \frac{1}{2}, \\ \widetilde{F}\left(2-2 t, x_{0}, Z\right) & \text { if } \frac{1}{2} \leq t \leq 1 .\end{cases}
$$

Clearly, $p_{*}\left([G]_{S^{1}}\right)=0$. That the assignment is well defined follows from the fact that if $F_{1}$ and $F_{2}$ are $S^{1}$-homotopic so will $\widetilde{F}_{1}$ and $\widetilde{F}_{2}$ be. To show that it is indeed a group morphism it suffices to note that the factor $t(1-t)$ in the definition of $\widetilde{\Phi}$ can be deformed to 1 and thus the addition is performed only on the invariant part of the maps. 
If $p_{*}\left([F]_{S^{1}}\right)=0$, then $F\left(t, x_{0}, 0\right)$ is deformable to $(1,0,0)$ and, by the $S^{1}$-equivariant Borsuk extension theorem, we may assume that $F\left(t, x_{0}, 0\right)=$ $(1,0,0)$. Since

$$
[\widetilde{F}]_{S^{1}}=\left[\left(f_{0}\left(t, x_{0}, 0\right), \Phi_{0}\left(t, x_{0}, 0\right), Z_{1}^{n_{1} / m_{0}}, \ldots, Z_{m}^{n_{m} / m_{0}}, 0, \ldots, 0\right)\right]_{S^{1}}
$$

(by deforming $Z$ to 0 in both $f_{0}$ and $\Phi_{0}$ ), then $[\widetilde{F}]_{S^{1}}=0_{S^{1}}$. This implies that the assignment is one to one. Let $\left(f_{0}, \Phi_{0}\right)$ induce an element in $\pi_{k}\left(S^{l}\right)$ (extended continuously to $[0,1] \times B_{0}$ and taking value $(1,0)$ at both $t=0$ and $t=1)$. Now let $H\left(t, x_{0}, Z\right)=\left(h_{0}, \Psi_{0}, \Psi\right)\left(t, x_{0}, Z\right)$ be a representative of an element in $\operatorname{ker} p_{*}$. As above, we may assume that $H\left(t, x_{0}, 0\right)=(1,0,0)$. Taking

$$
F\left(t, x_{0}, Z\right)=\left\{\begin{array}{l}
\left(f_{0}\left(2 t, x_{0}\right), \Phi_{0}\left(2 t, x_{0}\right),\right. \\
\left.2 t(1-2 t)\left(Z_{1}^{n_{m} / m_{0}}, \ldots, Z_{m}^{n_{m} / m_{0}}, 0, \ldots, 0\right)\right), \quad t \in\left[0, \frac{1}{2}\right], \\
H\left(2 t-1, x_{0}, Z\right), \quad t \in\left[\frac{1}{2}, 1\right],
\end{array}\right.
$$

it follows that $[F]_{S^{1}}=[H]_{S^{1}}+\left[\left(f_{0}, \Phi_{0}, Z_{1}^{n_{1} / m_{0}}, \ldots, Z_{m}^{n_{m} / m_{0}}, 0, \ldots, 0\right)\right]_{S^{1}}$. Moreover, $p_{*}\left([F]_{S^{1}}\right)=\left[\left(f_{0}, \Phi_{0}\right)\right]$, since $p_{*}\left([H]_{S^{1}}\right)=0$, and $[F]_{S^{1}}-[\tilde{F}]_{S^{1}}=$ $[H]_{S^{1}}$ by the associativity of the sum. Thus the assignment is onto. Q.E.D.

In [I] and [I.M.V] we introduced, for computational purposes, the $S^{1}$-equivariant map

$$
\beta: \mathbf{R}^{l} \times \mathbf{C}^{n} \rightarrow \mathbf{R}^{l} \times \mathbf{C}_{\diamond}^{n}
$$

defined as

$$
\beta\left(y_{0}, \xi_{1}, \ldots, \xi_{n}\right)=\left(y_{0}, \xi_{1}^{N^{\prime} / n_{1}}, \ldots, \xi_{n}^{N^{\prime} / n_{n}}\right)
$$

where $\mathbf{C}_{\diamond}^{n}$ stands for $\mathbf{C}^{n}$ with the $S^{1}$-action given by

$$
\mathrm{e}^{i N^{\prime} \varphi}\left(\xi_{1}, \ldots, \xi_{n}\right):=\left(e^{i N^{\prime} \varphi} \xi_{1}, \ldots, e^{i N^{\prime} \varphi} \xi_{n}\right) .
$$

As in the first part of this section, one can define the group $\pi_{k+2 m}^{S^{1}}\left(S^{l+2 n, \diamond}\right)$ (all the equivariant homotopy classes of $S^{1}$-maps with range in $\left.\mathbf{R} \times \mathbf{R}^{l} \times \mathbf{C}_{\diamond}^{n}\right)$ and it is easy to check that the map $\beta$ induces the group homomorphism

$$
\beta_{*}: \pi_{k+2 m}^{S^{1}}\left(S^{l+2 n}\right) \rightarrow \pi_{k+2 m}^{S^{1}}\left(S^{l+2 n, \diamond}\right)
$$

defined by

$$
\beta_{*}\left([F]_{S^{1}}\right)=[\beta \circ F]_{S^{1}} \text {. }
$$

After these preliminaries, we have the following commutative diagram.

$$
\begin{aligned}
& \pi_{k+2 m}^{S^{1}}\left(S^{l+2 n}\right) p_{*} \searrow \stackrel{\Theta^{*}}{\rightarrow} \swarrow_{*} \pi_{k+2 m, \cdot}^{S^{1}}\left(S^{l+2 n}\right) \\
& \beta * \downarrow \\
& \pi_{k+2 m}^{S^{1}}\left(S^{l+2 n, \diamond}\right) \\
& \nearrow_{*} \pi_{k}\left(S^{l}\right) \nwarrow p \text {. } \\
& \downarrow \beta \\
& \overrightarrow{\Theta^{*}} \quad \pi_{k+2 m, \cdot}^{S^{1}}\left(S^{l+2 n, \diamond}\right)
\end{aligned}
$$


In [I.M.V] we defined a degree existing in the group at the lower right corner. In the next section we will define an auxiliary degree taking values in the group at the upper right corner, and we shall completely determine the right half of the diagram when $m=1$ or $m \leq n+1-(k-l) / 2$. In order to do so we study the kernel of the homomorphism $p_{*}$. The next section will be devoted to this task.

4.2. The equivariant extension procedure (the study of $\operatorname{ker} p_{*}$ ). We shall consider here the question of extending $S^{1}$-equivariant maps (for a $S^{1}$-almost semifree action) without using the machinery of obstruction theory as developed in [I] and [I.M.V].

Let $I=[0,1], B_{0}=\left\{x_{0} \in \mathbf{R}^{k}:\left\|x_{0}\right\| \leq R_{0}\right\}$, and $B=\left\{Z \in \mathbf{C}_{.}^{m}:\|Z\| \leq R\right\}$. Until further notice, we will consider $S^{1}$-equivariant maps

$$
F: \partial\left(I \times B_{0} \times B\right) \rightarrow \mathbf{R} \times \mathbf{R}^{l} \times \mathbf{C}^{n} \backslash\{0\}
$$

of the particular form

$$
F\left(t, x_{0}, Z\right)=\left(f_{0}, \Phi_{0}, \Phi\right)\left(t, x_{0}, Z\right)
$$

with

$$
\begin{aligned}
f_{0}\left(t, x_{0}, \mathbf{e}^{i m_{0} \varphi} Z\right) & =f_{0}\left(t, x_{0}, e^{i m_{0} \varphi} Z_{1}, \ldots, e^{i m_{0} \varphi} Z_{m}\right) \\
& =f_{0}\left(t, x_{0}, Z_{1}, \ldots, Z_{m}\right), \\
\Phi_{0}\left(t, x_{0}, \mathbf{e}^{i m_{0} \varphi} Z\right) & =\Phi_{0}\left(t, x_{0}, e^{i m_{0} \varphi} Z_{1}, \ldots, e^{i m_{0} \varphi} Z_{m}\right) \\
& =\Phi_{0}\left(t, x_{0}, Z_{1}, \ldots, Z_{m}\right), \\
\Phi_{j}\left(t, x_{0}, \mathbf{e}^{i m_{0} \varphi} Z\right) & =\Phi_{j}\left(t, x_{0}, e^{i m_{0} \varphi} Z_{1}, \ldots, e^{i m_{0} \varphi} Z_{m}\right) \\
& =e^{i n_{j} \varphi} \Phi_{j}\left(t, x_{0}, Z_{1}, \ldots, Z_{m}\right)
\end{aligned}
$$

for $j=1, \ldots, n$.

Recall that due to the equivariance of $F$ the $n_{j}$ 's are multiples of $m_{0}$. Assume that $p_{*}\left([F]_{S^{1}}\right)=0$; that is, the invariant part of $F$,

$$
\left(f_{0}, \Phi_{0}\right): \partial\left(I \times B_{0}\right) \rightarrow \mathbf{R} \times \mathbf{R}^{l} \backslash\{0\},
$$

extends to a nonvanishing map $\left(\tilde{f}_{0}, \widetilde{\boldsymbol{\Phi}}_{0}\right): I \times B_{0} \rightarrow \mathbf{R} \times \mathbf{R}^{l} \backslash\{0\}$. Consider the map $\widetilde{F}_{1}: \partial\left(I \times B_{0} \times\{r \in \mathbf{R}: 0 \leq r \leq R\}\right) \rightarrow \mathbf{R} \times \mathbf{R}^{l} \times \mathbf{C}^{n} \backslash\{0\}$ defined by

$$
\widetilde{F}_{1}= \begin{cases}\left(\tilde{f}_{0}, \widetilde{\Phi}_{0}, 0\right) & \text { for } r=0, \\ F & \text { for } 0<r \leq R .\end{cases}
$$

Clearly, the map $\widetilde{F}_{1}$ gives an element $\left[\widetilde{F}_{1}\right]$ in the ordinary homotopy group $\pi_{k+1}\left(S^{l+2 n}\right)$ and $\widetilde{F}_{1}$ admits a nonvanishing extension to $I \times B_{0} \times\{r \in \mathbf{R}: 0 \leq$ $r \leq R\}$ if and only if $\left[\widetilde{F}_{1}\right]=0$ (one has always an extension if $k+1<l+2 n$ ).

Suppose now that $\left(\tilde{f}_{0}, \tilde{\Phi}_{0}, \widetilde{\Phi}\right)$ is such an extension of $\widetilde{F}_{1}$. Then we may define a $S^{1}$-equivariant extension $F_{1}$ of $\widetilde{F}_{1}$ to $I \times B_{0} \times\left(B \cap\left\{Z_{2}=\cdots=Z_{m}=0\right\}\right)$ 
by setting for $Z_{1}=e^{i \psi}\left|Z_{1}\right|$

$$
F_{1}\left(t, x_{0}, e^{i \psi}\left|Z_{1}\right|\right)=\left(\tilde{f}_{0}, \widetilde{\Phi}_{0}, \ldots, e^{i n_{j} / m_{0} \psi} \widetilde{\Phi}_{j}, \ldots\right)\left(t, x_{0},\left|Z_{1}\right|\right) \text {. }
$$

Note that $F_{1}\left(t, x_{0}, e^{i(\psi+2 k \pi)}\left|Z_{1}\right|\right)=F_{1}\left(t, x_{0}, e^{i \psi}\left|Z_{1}\right|\right)$ since $n_{j}$ is a multiple of $m_{0}$ for $j=1, \ldots, n$. Clearly, $F_{1}$ is a nonvanishing extension of $F$ to $I \times B_{0} \times\left(B \cap\left\{Z_{2}=\cdots=Z_{m}=0\right\}\right)$. Let us check that the map $F_{1}$ is $S^{1}$-equivariant. Indeed the $S^{1}$-action on the domain can be written as $\mathrm{e}^{i m_{0} \varphi}\left(t, x_{0}, Z_{1}\right)=\left(t, x_{0}, e^{i\left(m_{0} \varphi+\psi\right)}\left|Z_{1}\right|\right)$. Thus

$$
\begin{aligned}
F_{1}\left(\mathbf{e}^{i m_{0} \varphi}\left(t, x_{0}, Z_{1}\right)\right) & =\left(\tilde{f}_{0}, \widetilde{\Phi}_{0}, \ldots, e^{i\left(n_{j} / m_{0}\right)\left(m_{0} \varphi+\psi\right)} \tilde{\boldsymbol{\Phi}}_{j}, \ldots\right)\left(t, x_{0},\left|Z_{1}\right|\right) \\
& =\left(\tilde{f}_{0}, \tilde{\Phi}_{0}, \ldots, e^{i n_{j} \varphi} e^{i\left(n_{j} / m_{0}\right) \psi} \tilde{\Phi}_{j}, \ldots\right)\left(t, x_{0},\left|Z_{1}\right|\right) \\
& =\mathbf{e}^{i n \varphi} F_{1}\left(t, x_{0}, Z_{1}\right)
\end{aligned}
$$

Now suppose that the map $F$ has been extended $S^{1}$-equivariantly to $I \times B_{0} \times$ $\left(B \cap\left\{Z_{h}=Z_{h+1}=\cdots=Z_{m}=0\right\}\right)$ via the $S^{1}$-map $F_{h-1}\left(t, x_{0}, Z_{1}, \ldots, Z_{h-1}\right)$. Then on the topological sphere $\partial\left(I \times B_{0} \times\left(B \cap\left\{Z_{h+1}=\cdots=Z_{m}=0, Z_{h} \in \mathbf{R}\right.\right.\right.$, $\left.\left.0 \leq Z_{h} \leq R\right\}\right)$ ) one may consider the map $\widetilde{F}_{h}$ defined by

$$
\tilde{F}_{h}= \begin{cases}F_{h-1} & \text { on } I \times B_{0} \times\left(B \cap\left\{Z_{h}=Z_{h+1}=\cdots=Z_{m}=0\right\}\right), \\ F & \text { on the rest of the sphere. }\end{cases}
$$

Now the map $\widetilde{F}_{h}$ can be extended to the set $I \times B_{0} \times\left(B \cap\left\{Z_{h+1}=\cdots=Z_{m}=0\right.\right.$, $\left.\left.Z_{h} \in \mathbf{R}, 0 \leq Z_{h} \leq R\right\}\right)$ if it is homotopically trivial in $\pi_{k+2 h-1}\left(S^{l+2 n}\right)$. If this is the case and $\left(\tilde{f}_{0}, \widetilde{\Phi}_{0}, \widetilde{\Phi}\right)$ is a nonvanishing extension of $\widetilde{F}_{h}$, one obtains a $S^{1}$ equivariant extension $F_{h}$ of $F_{h-1}$ to the set $I \times B_{0} \times\left(B \cap\left\{Z_{h+1}=\cdots=Z_{m}=0\right\}\right)$ by setting

$$
\begin{aligned}
F_{h}\left(t, x_{0}, Z_{1}, \ldots, Z_{h-1}, e^{i \psi}\left|Z_{h}\right|\right) \\
\quad=\left(\tilde{f}_{0}, \widetilde{\Phi}_{0}, \ldots, e^{i\left(n_{j} / m_{0}\right) \psi} \widetilde{\Phi}_{j}, \ldots\right)\left(t, x_{0}, e^{-i \psi} Z_{1}, \ldots, e^{-i \psi} Z_{h-1},\left|Z_{h}\right|\right)
\end{aligned}
$$

for $Z_{h}=e^{i \psi}\left|Z_{h}\right|$. It is easy to see that the map $F_{h}$ is a $S^{1}$-equivariant extension of $F$ to the set $I \times B_{0} \times\left(B \cap\left\{Z_{h+1}=\cdots=Z_{m}=0\right\}\right)$, using as above the facts that $F_{h-1}$ is equivariant and $n_{j}$ is a multiple of $m_{0}$.

Clearly, the procedure just described may be repeated automatically as long as $k+2 h-1<l+2 n$, with $1 \leq h \leq m$, but it will require a particular consideration as soon as

$$
\begin{array}{ll}
h=h_{c}:=1 & \text { if } k-l \geq 2 n-1, \\
h=h_{c}:=n-\frac{k-l-1}{2} & \text { if }|k-l| \text { is odd, } \\
h=h_{c}:=n-\frac{k-l}{2}+1 & \text { if }|k-l| \text { is even } .
\end{array}
$$


Namely, we shall denote by $\operatorname{deg}_{K}(F)$ the ordinary homotopy class of the map $\widetilde{F}_{h_{c}}$ defined above, where $K$ stands for the kernel of $p_{*}$. Therefore,

$$
\operatorname{deg}_{K}(F) \in \begin{cases}\pi_{k+1}\left(S^{l+2 n}\right), & \text { if } k-l \geq 2 n-1\left(h_{c}=1\right), \\ \pi_{l+2 n}\left(S^{l+2 n}\right) \cong \mathbf{Z}, & \text { if }|k-l| \text { is odd } \\ \pi_{l+2 n+1}\left(S^{l+2 n}\right) \cong \mathbf{Z}_{2}, & \text { if }|k-l| \text { is even and } l+2 n>2, \\ \pi_{l+2 n+1}\left(S^{l+2 n}\right) \cong \mathbf{Z}, & \text { if }|k-l| \text { is even and } l+2 n=2, \\ \pi_{l+2 n+1}\left(S^{l+2 n}\right) \cong 0, & \text { if }|k-l| \text { is even and } l+2 n<2 .\end{cases}
$$

Note that the degree $\operatorname{deg}_{K}(F)$ depends a priori on the different extensions that one has to perform before arriving at the critical level $h_{c}$. That this is in fact not so will be proved below. First, we would like to point out some simple consequences of the above construction.

To this task let us introduce the following notation. Given a $S^{1}$-equivariant map $F=\left(f_{0}, \Phi_{0}, \Phi\right)$ let $\chi_{*}([F])$ denote the class of $F$ in $\pi_{k+2 m}\left(S^{l+2 n}\right)$, that is, when one forgets the group action. We are now in a position to prove the following result.

\section{Theorem 4.4.}

(i) If $1 \leq m<h_{c}$, where $h_{c}$ is defined by (4.6), then $p_{*}: \pi_{k+2 m, \cdot}^{S^{1}}\left(S^{l+2 n}\right) \rightarrow$ $\pi_{k}\left(S^{l}\right)$ is an isomorphism. Moreover, $\chi_{*}([F])=0$ provided that either $k \neq l$ or $m \neq n$, and $\chi_{*}([F])=\left(N^{\prime} / m_{0}^{n}\right) \operatorname{deg}\left(f_{0}, \Phi_{0}\right)$ if $k=l$ and $m=n$.

(ii) If $k=l$ and $m=n$, then the Brouwer degree of a $S^{1}$-equivariant map from $\partial D \subset \mathbf{R} \times \mathbf{R}^{k} \times \mathbf{C}^{m}$ into $\mathbf{R} \times \mathbf{R}^{l} \times \mathbf{C}^{n} \backslash\{0\}$ is equal to $\left(N^{\prime} / M^{\prime}\right) \operatorname{deg}\left(f_{0}, \Phi_{0}\right)$.

(iii) If $k=l$ and $m>n$, then $p_{*}\left([F]_{S^{1}}\right)=0$; that is, there are no $S^{1}$ equivariant maps defined on $\partial\left(I \times B_{0} \times B\right)$ with $\operatorname{deg}\left(f_{0}, \Phi_{0}\right) \neq 0$.

Proof. (i) Let $m<h_{c}$. Then any element in $\operatorname{ker} p_{*}$ extends to $I \times B_{0} \times B$; that is, $\operatorname{ker} p_{*}=\left\{[0]_{S^{1}}\right\}$. Moreover, if $k \geq l$ then, by (4.6), we have $h_{c} \leq$ $n+1$. Thus $m \leq n$ and by Lemma 4.3 the morphism $p_{*}$ is onto. If $k<l$ then $\pi_{k}\left(S^{l}\right)=0$. Therefore, we can conclude that $p_{*}$ is an isomorphism. Furthermore, $m<h_{c}$ implies $k+2 m \leq l+2 n$. Hence $\chi_{*}([F])=0$ except if the equality holds and $k \geq l$ (and hence $m \leq n$ ). But then, by Lemma 4.3, the map $F$ is $S^{1}$-homotopic to $\left(f_{0}, \Phi_{0}, Z_{1}^{n_{1} / m_{0}}, \ldots, Z_{m}^{n_{m} / m_{0}}, 0, \ldots, 0\right)$. If $m<$ $n$, then the Brouwer degree of this map is zero (make a deformation through the last components); that is, $\chi_{*}([F])=0$. In the case $m=n, \chi_{*}([F])=$ $\left(N^{\prime} / m_{0}^{m}\right) \operatorname{deg}\left(f_{0}, \Phi_{0}\right)$ by the product formula of the Brouwer degree.

(ii) Let $F: \partial D \subset \mathbf{R} \times \mathbf{R}^{k} \times \mathbf{C}^{m} \rightarrow \mathbf{R} \times \mathbf{R}^{l} \times \mathbf{C}^{n} \backslash\{0\}$ be $S^{1}$-equivariant. To compute the Brouwer degree of $F$, one can use the previous result applied to the map $\widetilde{F}\left(t, x_{0}, Z\right)=F\left(t, \Theta\left(x_{0}, Z\right)\right)$. Indeed, for $k=l$ and $m=n$, $\chi_{*}\left(\Theta^{*}[F]\right)=\left(N^{\prime} / m_{0}^{n}\right) \operatorname{deg}\left(f_{0}, \Phi_{0}\right)=\operatorname{deg}(\widetilde{F})=\left(M^{\prime} / m_{0}^{n}\right) \operatorname{deg}(F)$ (by the composition rule for degrees). Hence, $\operatorname{deg}(F)=\left(N^{\prime} / M^{\prime}\right) \operatorname{deg}\left(f_{0}, \Phi_{0}\right)$. 
(iii) Finally, for $k=l$ and $m>n$ one has that

$$
\chi_{*}([F])=\left(N^{\prime} / M^{\prime}\right) \operatorname{deg}\left(f_{0}, \Phi_{0}\right)=0
$$

on $I \times B_{0} \times\left(B \cap\left\{Z_{n+1}=\cdots=Z_{m}=0\right\}\right)$, by deforming the value of $F$ on the boundary of the former set to its value at the point $\left(t=\frac{1}{2}, x_{0}=0, Z=\right.$ $(0, \ldots, 0, R))$ through the map $F$ itself. Hence $p_{*}\left([F]_{S^{1}}\right)=0$. Q.E.D.

Remark 4.5. Part (ii) of Theorem 4.4 represents an extension of a result of Nirenberg [N].

In the following theorem we describe the main properties of $\operatorname{deg}_{K}(F)=\left[\widetilde{F}_{h_{c}}\right]$.

Theorem 4.6. $\operatorname{deg}_{K}: \operatorname{ker} p_{*} \rightarrow \pi_{k+2 h_{c}-1}\left(S^{l+2 n}\right)$ is a group homomorphism (in particular, it is independent of the previous extension) except in the following cases:

(a) $k=l-1, h_{c}=n+1$ and $N^{\prime}=m_{0}^{n}$;

(b) $k=l-1, h_{c}=n+1$, and $N^{\prime}>m_{0}^{n}$;

(c) $k \geq l-1, h_{c}=1$, and $n=0$;

(d) $|k-l|$ even, $h_{c}>1, k+l+n>1$, and $\sum_{j=1}^{n}\left(n_{j} / m_{0}\right)+h_{c}$ odd;

(e) $k=l=0$ and $n=1$.

Moreover, there is always an extension to the set $I \times B_{0} \times\left(B \cap\left\{Z_{h_{c+1}}=\cdots=\right.\right.$ $\left.Z_{m}=0\right\}$ ) if either (a) or (c) or (d) holds. If either (b) or (e) holds then $\operatorname{deg}_{K}$ is unique modulo $N^{\prime} / m_{0}^{n}$.

Proof. See Appendix D.

The following results describe some consequences of Theorems 4.4 and 4.6.

\section{Corollary 4.7.}

(i) If $h_{c}=1$ (thus $\left.k-l \geq 2 n-1\right)$, then

$$
\pi_{k+2 h_{c}}^{S^{1}},\left(S^{l+2 n}\right) \cong \begin{cases}0 & \text { if } n=0, \\ \pi_{k}\left(S^{l}\right) \times \pi_{k+1}\left(S^{l+2 n}\right) & \text { if } n>0 .\end{cases}
$$

(ii) If $k-l=2 n-1$, then

$$
\chi_{*}\left(\pi_{k+2 h_{c}, \cdot}^{S^{1}}\left(S^{l+2 n}\right)\right) \cong\left(\tilde{N} / m_{0}\right) \pi_{l+2 n+1}\left(S^{l+2 n}\right)
$$

where $\tilde{N}=\sum_{j=1}^{n} n_{j}(\tilde{N}=0$ if $n=0)$. More precisely, the restriction of $\chi_{*}$ to $\pi_{k}\left(S^{l}\right)$ has the following properties:

$$
\chi_{* \mid \pi_{k}\left(S^{l}\right)}= \begin{cases}\text { isomorphism, } & \text { if } n=1, n_{1} / m_{0} \text { is odd, and } l>2, \\ \text { onto, } & \text { if } n=1, n_{1} / m_{0} \text { is odd, and } l=2, \\ 0, & \text { otherwise, }\end{cases}
$$

while

$$
\chi_{* \mid \pi_{k+1}\left(S^{l+2 n}\right)}= \begin{cases}\text { isomorphism, } & \text { if } \tilde{N} / m_{0} \text { is odd and } l+2 n>2, \\ \text { has image }\left(n_{1} / m_{0}\right) \mathbf{Z}, & \text { if } \tilde{N} / m_{0} \text { is odd and } l=0, n=1, \\ 0, & \text { otherwise }\end{cases}
$$


(iii) If $h_{c}>1,|k-l|$ odd, and $n \geq 0$ then

$$
\pi_{k+2 h_{c},}^{S^{1}}\left(S^{l+2 n}\right) \cong \begin{cases}\pi_{k}\left(S^{l}\right) \times \mathbf{Z} & \text { if } k \neq l-1 \\ \mathbf{Z}_{N^{\prime} / m_{0}^{n}} & \text { if } k=l-1\end{cases}
$$

Furthermore,

$$
\chi_{*}\left(\pi_{k+2 h_{c}}^{S^{1}},\left(S^{l+2 n}\right)\right) \cong \begin{cases}0 & \text { for } \tilde{N} / m_{0}+h_{c} \text { odd }, \\ \mathbf{Z}_{2} & \text { for } \tilde{N} / m_{0}+h_{c} \text { even },\end{cases}
$$

whose generator is given by the element having $K$-degree equal to 1 , and

$$
\chi_{*}\left(\pi_{k}\left(S^{l}\right)\right) \cong \begin{cases}\mathbf{Z}_{2} & \text { if } N^{\prime} / m_{0}^{n} \text { is odd and } k=l+1 \geq 3, \\ 0 & \text { otherwise. }\end{cases}
$$

Here the isomorphisms are understood in the sense of Lemma 4.3.

Proof. See Appendix D.

Corollary 4.8. If $h_{c}>1,|k-l|$ even, and $n \geq 0$ then

$$
\pi_{k+2 h_{c},}^{S^{1}}\left(S^{l+2 n}\right) \cong \begin{cases}\mathbf{Z}_{\tilde{N} / m_{0}} & \text { if } n=1, k=l=0, \\ 0 & \text { if } k \leq l, k+l+n>1, \tilde{N} / m_{0}+h_{c} \text { odd, } \\ \pi_{k}\left(S^{l}\right) & \text { if } k>l, k+l+n>1, \tilde{N} / m_{0}+h_{c} \text { odd, } \\ \mathbf{Z} & \text { if } n=0, k=0, l=2, \tilde{N} / m_{0}+h_{c} \text { even, } \\ \mathbf{Z}_{2} & \text { if } k<l, k+l+n>2, \tilde{N} / m_{0}+h_{c} \text { even, } \\ \mathbf{Z}_{2} & \text { if } k=l, k+l+n>1, \tilde{N} / m_{0}+h_{c} \text { even, } \\ \mathbf{Z}_{2} \times \pi_{k}\left(S^{l}\right) & \text { if } k>l, \tilde{N} / m_{0}+h_{c} \text { even. }\end{cases}
$$

Furthermore,

$$
\chi_{*}\left(\pi_{k+2 h_{c}}^{S^{1}},\left(S^{l+2 n}\right)\right)= \begin{cases}0 & \text { if } \tilde{N} / m_{0}+h_{c} \text { is odd }, \\ \pi_{l+2 n+2}\left(S^{l+2 n}\right) \cong \mathbf{Z}_{2} & \text { if } \tilde{N} / m_{0}+h_{c} \text { is even } .\end{cases}
$$

If $\tilde{N} / m_{0}+h_{c}$ is even and $k>l$ then $\chi_{*}$ on $\pi_{k}\left(S^{l}\right)$ is 0 except if $k=l+2 \geq 4$, and the generator of the other component always gives the generator of $\mathbf{Z}_{2}$. Thus, except in the low-dimensional cases, i.e., if $n=1, k=l=0$ and if $n=0, k=0, l=2, \tilde{N} / m_{0}+h_{c}$ even, the morphisms $p_{*}$ and $\chi_{*}$ give all the information on $\pi_{k+2 h_{c}}^{S^{1}},\left(S^{l+2 n}\right)$. The isomorphisms are understood in the sense of Lemma 4.3.

Proof. See Appendix D.

4.3. Behavior of the $K$-degree under suspension and the range of $\operatorname{deg}_{K}(f, \Omega)$. If one has a one-dimensional suspension with trivial action, the $\Gamma$-equivariant suspension theorem (Theorem B in the Appendix) applies if the following dimensional inequalities hold:

(i) if $h_{c}=1$, then $k \leq 2 l-4$ for $n=0$ and $k \leq 2 l-2$ for $n \geq 1$; 
(ii) if $h_{c}>1$, then $k \leq 2 l-2$ except $h_{c}=2, n=0, l=k+2=2$ or 3 (for the details see Example B.3).

These conditions ensure that the suspension $\Sigma$ is actually an isomorphism. They are, however, too restrictive. We can weaken those assumptions and obtain the same result, using some facts presented in the previous section.

Since $p_{*} \circ \Sigma=\Sigma \circ p_{*}$ and $\chi_{*} \circ \Sigma=\Sigma \circ \chi_{*}$, we have that

$$
\operatorname{deg}_{K}(\Sigma F, \Sigma \widetilde{F})=\Sigma \operatorname{deg}_{K}(F, \widetilde{F}) \text {. }
$$

(See Lemma 0.3 for this notation.) Thus, if $1 \leq m \leq h_{c}$ one needs the condition $k \leq 2 l-2$ whenever $p_{*} \neq 0$, that is, if $m<h_{c}$, or $m=h_{c}=1 \leq n$ and $k \geq l$, or $h_{c}>1$ and $k>l$. On the other hand, $\Sigma$ is an isomorphism on $\operatorname{ker} p_{*}$ except if $h_{c}=1, k>2(2 n+l-1), n \geq 1$ and if either $n=0, k=0, l=2$ or $n=1, k=l=0$. In the last two cases $\Sigma$ is onto.

Similarly one can study the suspension $\Sigma_{r / m_{0}}: \mathbf{C} \rightarrow \mathbf{C}$ defined by $\Sigma(Z)=$ $Z^{r / m_{0}}$, where $r$ is a multiple of $m_{0}$ and the action on $\mathbf{C}$ is given by $\mathrm{e}^{i m_{0} \varphi}$. Hence, for $r=m_{0}$ the $\Gamma$-equivariant suspension theorem applies if $k \leq l+$ $2 n-2\left(k \geq 2\right.$ if $h_{c}>1$ and $|k-l|$ is even); see Example B.3. However, $p_{*} \Sigma_{r / m_{0}}=p_{*}$ and $\chi_{*} \Sigma_{r / m_{0}}=\left(r / m_{0}\right) \chi_{*} \Sigma_{1}=\left(r / m_{0}\right) \Sigma^{2} \chi_{*}$. Hence,

$$
\operatorname{deg}_{K}\left(\Sigma_{r / m_{0}} F, \Sigma_{r / m_{0}} \tilde{F}\right)=\frac{r}{m_{0}} \operatorname{deg}_{K}\left(\Sigma_{1} F, \Sigma_{1} \tilde{F}\right)=\frac{r}{m_{0}} \Sigma^{2} \operatorname{deg}_{K}(F, \tilde{F}) .
$$

Thus, if $1 \leq m<h_{c}$, then $h_{c}$ is increased by 1 under the suspension and so $\Sigma_{r / m_{0}}$ is an isomorphism.

Now let $m=h_{c}=1$. Then $\Sigma_{r / m_{0}}=0$, if $n=0$. On the other hand, $\Sigma_{r / m_{0}}\left(\pi_{k}\left(S^{l}\right) \times \pi_{k+1}\left(S^{l+2 n}\right)\right) \cong \pi_{k}\left(S^{l}\right) \times\left(r / m_{0}\right) \Sigma^{2} \pi_{k+1}\left(S^{l+2 n}\right)$. Note that $h_{c}=1$ also for the suspended problem. In particular, if $r=m_{0}$ and $k<2(2 n+l-1)$, then $\Sigma_{1}$ is an isomorphism.

If $|k-l|$ is odd and $k-l \leq 2 n$, then for $1 \leq h_{c}, h_{c}$ is transformed under the suspension in $h_{c}+1$, and we have that $\operatorname{deg}_{K}$ is uniquely defined in $Z$ if $k \neq l-1$. Hence

$$
\Sigma_{r / m_{0}}\left(\pi_{k}\left(S^{l}\right) \times \mathbf{Z}\right) \cong \pi_{k}\left(S^{l}\right) \times\left(r / m_{0}\right) \mathbf{Z}
$$

and

$$
\Sigma_{r / m_{0}}\left(\mathbf{Z}_{N / m_{0}^{n}}\right) \cong\left(r / m_{0}\right) \mathbf{Z}_{N r / m_{0}^{n+1}}
$$

giving a one-to-one map and an isomorphism if $r=m_{0}$.

If $|k-l|$ is even and $k-l \leq 2 n$, then for $1 \leq h_{c}, h_{c}$ is transformed under the suspension into $h_{c}+1$ and $\tilde{N} / m_{0}+h_{c}$ will conserve its parity if and only if $r / m_{0}$ is odd. In the case when $r / m_{0}$ is even, then $\operatorname{deg}_{K}\left(\Sigma_{r / m_{0}} F, \Sigma_{r / m_{0}} \widetilde{F}\right)=0$ (since it belongs to $Z_{2}$ if $l+2 n \geq 1$ and if $l=n=0$ then $h_{c}=1$ and so the equivariant group reduces to 0$)$. Then $\Sigma_{r / m_{0}}$ is 0 on $\operatorname{ker} p_{*}$ and an isomorphism on $\operatorname{Im} p_{*}$. Hence $\Sigma_{r / m_{0}}$ is never onto (unless in the trivial case) and it is one to one only if $k>l$ and $\tilde{N} / m_{0}+h_{c}$ is odd. In particular, $\chi_{*} \Sigma_{r / m_{0}}=0$. 
We now examine the case $r / m_{0}$ odd. If $l+2 n>2$, then

$$
\operatorname{deg}_{K}\left(\Sigma_{r / m_{0}} F, \Sigma_{r / m_{0}} \widetilde{F}\right)=\operatorname{deg}_{K}(F, \widetilde{F}),
$$

thus $\Sigma_{r / m_{0}}$ is an isomorphism. If $n=0, k=l=0,1$, or 2 , then $\Sigma_{r / m_{0}}=0$ (since $h_{c}=1$, the first group is trivial). If $n=0, k=0, l=2$, then $\Sigma_{r / m_{0}}$ is onto $\mathbf{Z}_{2}$ but it will kill all the maps with $K$-degree even. Finally, if $n=1$, $k=l=0$, then $\Sigma_{r / m_{0}}=0$ if $n_{1} / m_{0}$ is odd and it is onto $\mathbf{Z}_{2}$ if $n_{1} / m_{0}$ is even (one to one if $n_{1}=2 m_{0}$ ), killing all even integers in $Z_{n_{1} / m_{0}}$. In all cases, one has that $\chi_{*} \Sigma_{r / m_{0}}=\chi_{*}$.

Thus a direct study of the groups improves the $\Gamma$-suspension theorem. Namely, one also obtains an isomorphism in the following cases: $h_{c}=1$, $k<2(2 n+l-1) ; k-l=2 n-1 ; k-l=2 n$ and $k \geq 3$ (not covered at all by the $\Gamma$-suspension theorem); $h_{c}>1,|k-l|$ even, and $k \geq 1$. Furthermore, one has a precise knowledge of the behavior of the suspension.

We would like to add in passing that one gets into the stable range after a finite number of suspensions.

We shall now study the range of $\operatorname{deg}_{K}(f, \Omega)$. Let $\Omega$ be an open bounded invariant subset of $\mathbf{R}^{k} \times \mathbf{C}^{m}$. and assume that $m \leq h_{c}$. Which elements of $\pi_{k+2 m,}^{S^{1}}\left(S^{l+2 n}\right)$ are realized as the $S^{1}$-degree of equivariant maps $f: \bar{\Omega} \rightarrow \mathbf{R}^{l} \times$ $\mathrm{C}^{n}$ such that $f \neq 0$ on $\partial \Omega$ ?

Let us recall that an equivariant map $f$, as above, gives rise to a $S^{1}$-map $F$ of the form

$$
\left(f_{0}, \Phi_{0}, \Phi\right)\left(t, x_{0}, Z\right)=\left(2 t+2 \varphi\left(x_{0}, Z\right)-1, \Phi_{0}\left(x_{0}, Z\right), \Phi\left(x_{0}, Z\right)\right) .
$$

Theorem 4.9. Let $\Omega \neq \varnothing$ and $m \leq h_{c}$. Then we have the following conclusions:

(i) If $\bar{\Omega} \cap\{Z=0\}=\varnothing$, then $p_{*}\left[\left(2 t+2 \varphi-1, \Phi_{0}, \Phi\right)\right]=0$. Thus any element in $\operatorname{ker} p_{*}$ is obtained, except:

(a) if $h_{c}=1$, then any suspension in $\pi_{k+1}\left(S^{l+2 n}\right)$ is attained (hence any element if $k \leq 2 l+4 n-3)$;

(b) if either $n=0, k=0, m=2, l=2$ or $n=1, k=l=0, m=2$, then, in general, the $S^{1}$-degree is trivial.

(ii) If $\bar{\Omega} \cap\{Z=0\} \neq \varnothing$, then any element in $\pi_{k+2 m,}^{S^{1}}\left(S^{l+2 n}\right)$ is obtained as the $S^{1}$-degree of an equivariant map on $\bar{\Omega}$, with the following exceptions:

(a) for the elements in $\operatorname{ker} p_{*}$ the same exceptions as in (i) and if $l=0$ then the $S^{1}$-degree is not defined;

(b) for the elements in $\operatorname{Im} p_{*}$, any suspension is achieved (hence any element if either $k \leq 2 l-2$ or $k=l=0$ and $m<h_{c}$ ).

In Appendix $\mathrm{D}$ we shall give concrete generators of the degree and the proof of this theorem.

Remark 4.10. Thus, according to Lemma 4.3, $\operatorname{deg}_{S^{1}}\left(\left(\Phi_{0}, \Phi\right), \Omega\right)$ has two components:

$$
\left.\operatorname{deg}\left(\Phi_{0}\left(x_{0}, 0\right), \Omega \cap\{Z=0\}\right)\right),
$$


which is always trivial if $\Omega \cap\{Z=0\}=\varnothing$, and

$$
\operatorname{deg}_{K}\left(g\left(t, x_{0}, Z\right),(0,1) \times B\right),
$$

where $B$ is a ball containing $\Omega$ and

$$
g\left(t, x_{0}, Z\right)=\left\{\begin{array}{l}
\left(2 t+2 \varphi(Y)-1, \Phi_{0}(Y),(1-2 t) \Phi(Y)\right), \\
\left(2(1-t)+2 \varphi(Y)-1, \Phi_{0}(Y),\right. \\
\left.\quad(2 t-1)\left(Z_{1}^{n_{1} / m_{0}}, \ldots, Z^{n_{m} / m_{0}}, 0, \ldots, 0\right)\right),
\end{array} \quad t \in\left[0, \frac{1}{2}, 1\right] .\right.
$$

Here, $Y=\left(x_{0}, Z\right)$. As in Remark A.5, it is easy to see that

$$
\begin{aligned}
& {[g]_{S^{1}}=\left[\left(f_{0}, \Phi_{0}, \Phi\right)\right]_{S^{1}}-\left[\left(f_{0}, \Phi_{0}, Z_{1}^{n_{1} / m_{0}}, \ldots, Z_{m}^{n_{m} / m_{0}}, 0, \ldots, 0\right)\right]_{S^{1}},} \\
& m \leq h_{c} \leq n,
\end{aligned}
$$

whenever $p_{*}\left([F]_{S^{1}}\right) \neq 0$. If $p_{*}\left([F]_{S^{1}}\right)=0$, then one has only the $K$-degree of $F$. Note that the zeros of $g$ are for $t=\frac{1}{2}, \Phi_{0}(Y)=0$, and $Y \in \Omega \cup N$ (with $\varphi(Y)=0)$.

We would like to point out that if $n \geq h_{c}=m$ and $\Phi_{0}^{-1}(0) \cap \Omega^{S^{1}} \neq \varnothing$, then $\operatorname{deg}_{S^{1}}(f, \Omega)$ can be computed as follows.

Since $f \neq 0$ on $\partial \Omega$ and $\Phi\left(x_{0}, 0\right)=0$, there exists $\varepsilon>0$ such that $\Phi_{0}\left(x_{0}, Z\right) \neq 0$ when $\left(x_{0}, Z\right) \in \partial \Omega$ and $|Z| \leq 2 \varepsilon$. Now let $\psi: \mathbf{R}^{+} \rightarrow[0,1]$ be a continuous function such that $\psi(r)=1$ if $0 \leq r \leq \varepsilon$ and $\psi(r)=0$ if $r \geq 2 \varepsilon$. Clearly the $S^{1}$-homotopy

$$
\begin{aligned}
h_{\tau}\left(x_{0}, Z\right)=( & \Phi_{0}\left(x_{0}, Z\right),(1-\tau \psi(|Z|)) \Phi\left(x_{0}, Z\right) \\
& \left.+\tau \psi(|Z|)\left(Z_{1}^{n_{1} / m_{0}}, \ldots, Z_{m}^{n_{m} / m_{0}}, 0, \ldots, 0\right)\right),
\end{aligned}
$$

$\tau \in[0,1]$, is admissible and such that $h_{1}\left(x_{0}, Z\right) \neq 0$ if $|Z|=\varepsilon$. Thus, by the homotopy and additivity property of the $S^{1}$-degree, we have that

$$
\begin{aligned}
\operatorname{deg}_{S^{1}}(f, \Omega)= & \operatorname{deg}_{S^{1}}\left(h_{1}, \Omega\right)=\operatorname{deg}_{S^{1}}\left(h_{1}, \Omega \cap\{|Z|<\varepsilon\}\right) \\
& +\operatorname{deg}_{S^{1}}\left(h_{1}, \Omega \cap\{|Z|>\varepsilon\}\right) .
\end{aligned}
$$

Now, by the excision property of the $S^{1}$-degree, we obtain that

$$
\begin{aligned}
\operatorname{deg}_{S^{1}} & \left(h_{1}, \Omega \cap\{|Z|<\varepsilon\}\right)=\operatorname{deg}_{S^{1}}\left(h_{1}, \Omega^{S^{1}} \times\{|Z|<\varepsilon\}\right) \\
& =\operatorname{deg}_{S^{1}}\left(\left(\Phi_{0}\left(x_{0}, 0\right), Z_{1}^{n_{1} / m_{0}}, \ldots, Z_{m}^{n_{m} / m_{0}}, 0, \ldots, 0\right), \Omega^{S^{1}} \times\{|Z|<\varepsilon\}\right) \\
& =\left(\operatorname{deg}\left(\Phi_{0}\left(x_{0}, 0\right), \Omega^{S^{1}}\right), 0\right) .
\end{aligned}
$$

The last two equalities are obtained by first deforming $Z$ to 0 in $\Phi_{0}\left(x_{0}, Z\right)$ and then applying Lemma 4.3 directly to compute the $K$-degree of $h_{1}$ on the set $\Omega \cap\{|Z|<\varepsilon\}$. On the other hand, we have that

$$
\operatorname{deg}_{S^{1}}\left(h_{1}, \Omega \cap\{|Z|>\varepsilon\}\right)=\left(0, \operatorname{deg}_{K}\left(h_{1}, \Omega \cap\{|Z|>\varepsilon\}\right)\right),
$$

since $\operatorname{deg}_{S^{1}}\left(h_{1}, \Omega \cap\{|Z|>\varepsilon\}\right)=[g]_{S^{1}}$, where $g\left(t, x_{0}, Z\right)=\left(2 t+2 \varphi\left(x_{0}, Z\right)-\right.$ $\left.1, h_{1}\left(x_{0}, Z\right)\right)$ with $\varphi\left(x_{0}, Z\right)=1$ for $|Z| \leq \varepsilon$. Thus,

$$
\operatorname{deg}_{S^{1}}(f, \Omega)=\left(\operatorname{deg}\left(\Phi_{0}, \Omega^{S^{1}}\right), 0\right)+\left(0, \operatorname{deg}_{K}\left(h_{1}, \Omega \cap\{|Z|>\varepsilon\}\right)\right) .
$$


If $m=h_{c}>n$, then $k<l$ and hence $p_{*}\left([F]_{S^{1}}\right)=0$; thus one has only the $K$-degree of $F$.

4.4. The infinite-dimensional case. The simplest situation perhaps arises when the action of $S^{1}$ is the same both on $\mathbf{R}^{M}$ and $\mathbf{R}^{N}$, in the sense that $m=n$ and $k=l+\tau$, where $\tau$ is the number of free parameters in the problem.

If one wishes a topological invariant which is not completely determined by the invariant part, then it is necessary to consider the case when $m=h_{c}$ and $n>1$, if infinite-dimensional problems are considered. This immediately reduces the choice to either $k=l+1$ or $k=l+2$.

Let us consider first the case when $k=l+2$. Then in order to have a nontrivial invariant, i.e., an element in $\operatorname{ker} p_{*}$, the number $\tilde{N} / m_{0}+h_{c}$ has to be even. Moreover, the morphism $\chi_{*}$ turns out to be onto and sends the generator of $\operatorname{ker} p_{*}$ into the generator of $\pi_{k+2 n}\left(S^{l+2 n}\right)$. Thus, in this situation, there is no gain in taking into account the $S^{1}$-action. Moreover, the "suspension" $\Sigma_{r}$ is an isomorphism provided that $r$ is odd (taking $m_{0}=1$ ); thus all the $n_{j}$ 's have to be odd, in which case $\tilde{N}+n$ is even.

Let us now consider the case when $k=l+1$. Then if $n>0$, we have that $\pi_{k+2 h_{c}, \cdot}^{S^{l}}\left(S^{l+2 n}\right) \cong \pi_{l+1}\left(S^{l}\right) \times \mathbf{Z}$ and the $K$-degree is well defined. Moreover, the suspension $\Sigma$ is an isomorphism if $l \geq 3$ and its restriction to $\operatorname{ker} p_{*}$ is an isomorphism, provided that $n>0$. Furthermore, $\Sigma_{r / m_{0}}$ is an isomorphism if $r=m_{0}$ and $n>0$ (notice that $\Sigma_{r / m_{0}}$ is one to one if $r$ is a multiple of $\left.m_{0}\right)$. Finally, $\chi_{*}([F]) \neq 0$ if and only if $[F]_{S^{1}}$ belongs to $\operatorname{ker} p_{*}$ and $F$ has an odd $K$-degree with $\tilde{N} / m_{0}+h_{c}$ even (for example, if $n_{j} / m_{0}$ is odd for all $j$ 's). In this case the $K$-degree will detect nontrivial elements, which cannot be obtained if one forgets the $S^{1}$-action.

We shall now discuss the problem of computing the $S^{1}$-degree in the infinitedimensional setting. Let $\mathbf{R}^{N}, \mathbf{R}^{N+1}$, and $E$ be $S^{1}$-spaces. We shall assume that $\left(\mathbf{R}^{N}\right)^{S^{1}}=\mathbf{R}^{l},\left(\mathbf{R}^{N+1}\right)^{S^{1}}=\mathbf{R}^{l+1}$, and $E$ is an infinite-dimensional Banach space. Moreover, from now on we shall write any element of the $S^{1}$-space $\mathbf{R}^{N} \times E \times \mathbf{R}$ as $(x, \nu)$ with $x \in \mathbf{R}^{N} \times E$ and $\nu \in \mathbf{R}$, which is considered as a one-dimensional subspace of $\mathbf{R}^{l+1}$.

Let $\Omega$ be an open bounded invariant subset of $\mathbf{R}^{N} \times E \times \mathbf{R}$ and let $f: \bar{\Omega} \rightarrow$ $\mathbf{R}^{N} \times E$ be a $S^{1}$-equivariant compact map such that

$$
x-f(x, \nu) \neq 0 \quad \text { for any }(x, \nu) \in \partial \Omega .
$$

(Note that the first $N$ components of the map $f$ will be taken of the form $x_{N}-f_{N}\left(x_{N}, y, \nu\right)$ so that the map $x-f(x, \nu)$ will have as first components $f_{N}\left(x_{N}, y, \nu\right)$, since the actions on $\mathbf{R}^{N+1}$ and $\mathbf{R}^{N}$ may be different.)

The approximation procedure. We shall assume that the $\Gamma$-equivariant suspension theorem is valid (for details see Example B.4 in the Appendix). Thus, 
following $\S 3$, let us denote by $\left(E_{n}\right)_{n \in \mathbf{N}}$ a sequence of finite-dimensional subspaces of $\mathbf{R}^{N} \times E \times \mathbf{R}$ such that $\mathbf{R}^{N+1} \subset E_{n}$ for all $n$, and let $E_{n}=\left(E_{n}\right)^{S^{1}} \times$ $\widetilde{E}_{n}$, with $\operatorname{dim} \widetilde{E}_{n}=n$, so that any element of $E_{n}$ will be written as $x_{n}=$ $\left(x_{0}, z_{1}, \ldots, z_{n}, \nu\right)$. Then we have that

$$
\operatorname{deg}_{S^{1}}(x-f(x, \nu), \Omega)=\operatorname{deg}_{S^{1}}\left(x_{n}-f_{n}\left(x_{n}, \nu\right), \Omega_{n}\right)
$$

for $n$ large enough, where $\Omega_{n}=\Omega \cap E_{n}$ and $f_{n}$ is a $S^{1}$-approximation of $f$ with values in $E_{n}$.

For each $n$ large enough, let $\Theta_{n, n_{0}}^{*}$ be the morphism induced by the map

$$
\Theta_{n, n_{0}}\left(x_{0}, Z_{1}, \ldots, Z_{n}, \nu\right)=\left(x_{0}, Z_{1}^{m_{1} / n_{0}}, \ldots, Z_{n}^{m_{n} / n_{0}}, \nu\right)
$$

(see $\S 4.1)$.

The $S^{1}$-action on the $Z_{i}$ 's is given by $\mathrm{e}^{i n_{0} \varphi}$ with $n_{0}$ a common divisor of $m_{1}, \ldots, m_{n}$.

We would like to point out that the map $\Theta_{n, n_{0}}$ is not of the form Id-compact when considered between infinite-dimensional spaces. Hence one cannot do directly the reduction of a $S^{1}$-action to an almost semifree action without passing first to the finite-dimensional setting.

Using Lemma 4.3, we now have that

$$
\Theta_{n, n_{0}}^{*} \operatorname{deg}_{S^{1}}\left(x_{n}-f_{n}\left(x_{n}, \nu\right), \Omega_{n}\right)=\operatorname{deg}_{S^{1}}\left(G_{n}, \Theta_{n, n_{0}}^{-1}(\Omega)\right),
$$

where the $i$ th component of the map $G_{n}$ is given by

$$
G_{n, i}=Z_{i}^{m_{i} / n_{0}}-f_{n, i}\left(x_{0}, Z_{1}^{m_{1} / n_{0}}, \ldots, Z_{n}^{m_{n} / n_{0}}, \nu\right) \text {; }
$$

here $f_{n, i}$ denotes the $i$ th component of $f_{n}, i=0, \ldots, n$, and $Z_{0}$ stands for $x_{0}$ with exponent $1\left(m_{0}=n_{0}\right)$.

Moreover, for $E_{n} \subset E_{m}$, we shall write

$$
x_{m}=\left(x_{0}, Z_{1}, \ldots, Z_{n}, y_{0}, Z_{n+1}, \ldots, Z_{m}, \nu\right)
$$

and we shall also have that

$$
\boldsymbol{\Theta}_{m, m_{0}}^{*} \operatorname{deg}_{S^{1}}\left(x_{m}-f_{m}\left(x_{m}, \nu\right), \Omega_{m}\right)=\operatorname{deg}_{S^{1}}\left(H_{m}, \Theta_{m, m_{0}}^{-1}(\Omega)\right),
$$

where the $i$ th component of the map $H_{m}$ is given by

$$
H_{m, i}=\xi_{i}^{m_{i} / m_{0}}-f_{m, i}\left(\xi_{0}, \xi_{1}^{m_{1} / m_{0}}, \ldots, \xi_{m}^{m_{m} / m_{0}}, \nu\right), \quad i=0, \ldots, m .
$$

(Not necessarily $m_{0}=n_{0}$, but if we choose $n_{0}$ to be the greatest common divisor of $m_{1}, \ldots, m_{n}$ then $m_{0}$ divides $n_{0}$, hence one may take $n_{0}$ to be $m_{0}$.) Here the $S^{1}$-action on the $\xi_{i}$ 's is given by $\mathrm{e}^{i m_{0} \varphi}$.

However, by the $S^{1}$-equivariant suspension theorem

$$
\operatorname{deg}_{S^{1}}\left(x_{m}-f_{m}\left(x_{m}, \nu\right), \Omega_{m}\right)=\operatorname{deg}_{S^{1}}\left(x_{n}-f_{n}\left(x_{n}, \nu\right), \Omega_{n}\right) .
$$

In fact, the map $x_{m}-f_{m}\left(x_{m}, \nu\right)$ is equivariantly deformable relative to $\partial \Omega_{n}$ to the map $\left(x_{n}-f_{n}\left(x_{n}, \nu\right), x_{m-n}\right)$ relative to $\partial\left(\Omega_{n} \times\left\{x_{m-n}:\left\|x_{m-n}\right\|<\varepsilon\right\}\right)$, 
where $x_{m-n}=\left(y_{0}, z_{n+1}, \ldots, z_{m}\right)$. Let $U=\Theta_{n, n_{0}}^{-1}(\Omega) \times\left\{\left|y_{0}\right|<\varepsilon\right\} \times\left\{\left|\xi_{i}\right|<\right.$ $\varepsilon: i=n+1, \ldots, m\}$ and

$$
\begin{aligned}
H=\left(\xi_{0}^{m_{0} / m_{0}}-f_{n, 0}\left(\xi_{0}, \ldots, \xi_{n}^{m_{n} / m_{0}}, \nu\right), \ldots, \xi_{n}^{m_{n} / m_{0}}\right. & \\
& \left.\quad-f_{n, n}\left(\xi_{0}, \ldots, \xi_{n}^{m_{n} / m_{0}}, \nu\right), y_{0}, \xi_{n+1}^{m_{n+1} / m_{0}}, \ldots, \xi_{m}^{m_{m} / m_{0}}\right) .
\end{aligned}
$$

Thus,

$$
\begin{aligned}
\Theta_{m, m_{0}}^{*} \operatorname{deg}_{S^{1}}\left(x_{m}-f_{m}\left(x_{m}, \nu\right), \Omega_{m}\right) & =\operatorname{deg}_{S^{1}}(H, U) \\
=\left(\operatorname{deg}\left(\left(x_{0}-f_{n, 0}\left(x_{0}, \nu\right), y_{0}\right), \Omega \cap\left(\mathbf{R}^{N} \times E_{m} \times \mathbf{R}\right)^{S^{1}}\right),\right. & \left.\operatorname{deg}_{K, m_{0}}\left(g_{m}\left(t, x_{0}, y_{0}, \xi, \nu\right)\right)\right),
\end{aligned}
$$

where

$$
g_{m}\left(t, x_{0}, y_{0}, \xi, \nu\right)
$$

$$
\left\{\begin{array}{c}
2 t+2 \varphi\left(x_{0}, y_{0}, \xi, \nu\right)-1 \\
x_{0}-f_{n, 0}\left(x_{0}, \xi_{1}^{m_{1} / m_{0}}, \ldots, \xi_{n}^{m_{n} / m_{0}}\right) \\
y_{0} \\
(1-2 t)\left(\xi_{1}^{m_{1} / m_{0}}-f_{n, 1}\left(x_{0}, \xi_{1}^{m_{1} / m_{0}}, \ldots, \xi_{n}^{m_{n} / m_{0}}\right)\right) \\
\vdots \\
(1-2 t)\left(\xi_{n}^{m_{n} / m_{0}}-f_{n, n}\left(x_{0}, \xi_{1}^{m_{1} / m_{0}}, \ldots, \xi_{n}^{m_{n} / m_{0}}\right)\right) \\
(1-2 t) \xi_{n+1}^{m_{n+1} / m_{0}} \\
\vdots \\
(1-2 t) \xi_{m}^{m_{m} / m_{0}} \\
(2 t-1) \xi_{1}^{m_{1} / m_{0}} \\
\vdots \\
(2 t-1) \xi_{m}^{m_{m} / m_{0}} \\
y_{0}-f_{n, 0}\left(x_{0}, \xi_{1}^{m_{1} / m_{0}}, \ldots, \xi_{n}^{m_{n} / m_{0}}\right) \\
\left(x_{0}, y_{0}, \xi, \nu\right)-1
\end{array}\right)^{\mathrm{tr}} \quad 0 \leq t \leq \frac{1}{2},
$$

Here $(, \ldots,)^{\mathrm{tr}}$ denotes the transposed vector and

$$
\varphi\left(x_{0}, y_{0}, \xi, \nu\right)=\varphi\left(x_{0}, y_{0}, \xi_{1}^{m_{1} / m_{0}}, \ldots, \xi_{m}^{m_{m} / m_{0}}, \nu\right),
$$

as in the proof of Lemma 4.3. (Recall that, if $\xi_{i} \in \mathbf{R}^{M}, i \leq n$, then $\xi_{i}^{m_{i} / m_{0}}-$ $f_{n, i}=g_{n, i}$ with the $S^{1}$-action $e^{i n_{i} \varphi}$ and $\xi_{i}^{m_{i} / m_{0}}$ has to be interpreted as $\xi_{i}^{n_{i} / n_{0}}$ for these $i$ 's.)

Now, if $\operatorname{dim}\left(\mathbf{R}^{N} \times E\right)^{S^{1}} \leq 2$, then we have (by the choice of the $E_{n}$ 's) that $y_{0}=0$; thus the invariant part is unchanged. If $\operatorname{dim}\left(\mathbf{R}^{N} \times E\right)^{S^{1}}>2$ and $y_{0} \neq 0$, 
then one is in the stable range. Hence

$$
\begin{aligned}
\operatorname{deg}\left(\left(x_{0}-f_{n, 0}\left(x_{0}, 0, \nu\right), y_{0}\right), \Omega_{m}^{S^{1}}\right) & =\operatorname{deg}\left(x_{0}-f_{n, 0}\left(x_{0}, 0, \nu\right), \Omega_{n}^{S^{1}}\right) \\
& =\operatorname{deg}\left(x-f(x, \nu), \Omega^{S^{1}}\right) .
\end{aligned}
$$

Moreover, using a linear deformation, the $i$ th component, $i=1, \ldots, m$, in $g_{m}$ can be deformed to

$$
\left\{\begin{array}{c}
\left(\begin{array}{c}
\xi_{1}^{m_{1} / m_{0}}-(1-2 t) f_{n, 1}\left(x_{0}, \xi_{1}^{m_{1} / m_{0}}, \ldots, \xi_{n}^{m_{n} / m_{0}}\right) \\
\xi_{n}^{m_{n} / m_{0}}-(1-2 t) f_{n, n}\left(x_{0}, \xi_{1}^{m_{1} / m_{0}}, \ldots, \xi_{n}^{m_{n} / m_{0}}\right) \\
\xi_{n+1}^{m_{n+1} / m_{0}} \\
\vdots \\
\xi_{m}^{m_{m} / m_{0}}
\end{array}\right)^{\mathrm{tr}}, \quad 0 \leq t \leq \frac{1}{2}, \\
\left(\begin{array}{c}
\xi_{1}^{m_{1} / m_{0}} \\
\vdots \\
\xi_{m}^{m_{m} / m_{0}}
\end{array}\right)^{\mathrm{tr}}, \quad \frac{1}{2} \leq t \leq 1
\end{array}\right.
$$

We may also deform the map $\varphi$ to $\varphi\left(x_{0}, 0, \xi_{1}^{m_{1} / m_{0}}, \ldots, \xi_{n}^{m_{n} / m_{0}}, 0, \nu\right)$. Now the component $y_{0}$ acts as a trivial suspension (which is always an isomorphism on $\left.\operatorname{ker} p_{*}\right)$ and $Z_{i}^{m_{i} / m_{0}}$ acts as an $\left(m_{i} / m_{0}\right)$ th suspension for $i=n+1, \ldots, m$. Thus,

$$
\operatorname{deg}_{K, m_{0}}\left(g_{m}\left(t, x_{0}, y_{0}, \xi, \nu\right)\right)=\prod_{i=n+1}^{m} \frac{m_{i}}{m_{0}} \operatorname{deg}_{K, m_{0}}\left(g_{n}\left(t, x_{0}, 0, \xi_{1}, \ldots, \xi_{n}, 0, \nu\right)\right)
$$

(in order to derive the above identity, we have used the product theorem for the Brouwer topological degree; this explains the orientation we have chosen). Moreover, using Remark D.6, it follows that

$$
\begin{aligned}
\operatorname{deg}_{K, 1} & \left(g_{n}\left(t, x_{0}, 0, \xi_{1}, \ldots, \xi_{n}, 0, \nu\right)\right) \\
& =m_{0}^{n-1} \operatorname{deg}_{K, m_{0}} g_{n}\left(\left(t, x_{0}, 0, \xi_{1}, \ldots, \xi_{n}, 0, \nu\right)\right) .
\end{aligned}
$$

Therefore,

$$
\begin{aligned}
& \Theta_{m, m_{0}}^{*} \operatorname{deg}_{S^{1}}\left(x_{m}-f_{m}\left(x_{m}, \nu\right), \Omega_{m}\right) \\
& =\left(\operatorname{deg}\left(x-f(x, \nu), \Omega^{S^{1}}\right),\right. \\
& \left.\quad m_{0}^{-m+1} \prod_{i=n+1}^{m} m_{i} \operatorname{deg}_{K, 1}\left(g_{n}\left(t, x_{0}, 0, \xi_{1}, \ldots, \xi_{n}, 0, \nu\right)\right)\right), \\
& \Theta_{n, n_{0}}^{*} \operatorname{deg}_{S^{1}}\left(x_{n}-f_{n}\left(x_{n}, \nu\right), \Omega_{n}\right) \\
& =\left(\operatorname{deg}\left(x-f(x, \nu), \Omega^{S^{1}}\right), n_{0}^{-n+1} \operatorname{deg}_{K, 1}\left(g_{n}\left(t, x_{0}, 0, \xi_{1}, \ldots, \xi_{n}, 0, \nu\right)\right)\right) .
\end{aligned}
$$


Definition 4.11. We shall call the normalized $S^{1}$-degree of the map $x-f(x, \nu)$ relative to $\Omega$, denoted by $\operatorname{deg}_{S^{1}}^{N}(x-f(x, \nu), \Omega)$, the pair

$$
\begin{array}{r}
\left(\operatorname{deg}\left(x-f(x, \nu), \Omega^{S^{1}}\right), n_{0}^{n-1}\left(\prod_{1}^{n} m_{i}\right)^{-1} \operatorname{deg}_{K, n_{0}}\left(g_{n}\left(t, x_{0}, \xi_{1}, \ldots, \xi_{n}, \nu\right)\right)\right) \\
\in \pi_{l+1}\left(S^{l}\right) \times \mathbf{Q} .
\end{array}
$$

Here $g_{n}\left(t, x_{0}, \xi_{1}, \ldots, \xi_{n}, \nu\right)$ is defined through the map

$$
\left\{\begin{aligned}
\left(2 t+2 \varphi\left(x_{0}, z, \nu\right)-1, x_{0}-f_{0}\left(x_{0}, z, \nu\right),\right. & \\
\left.z-(1-2 t) f_{\infty}\left(x_{0}, z, \nu\right)\right), & 0 \leq t \leq \frac{1}{2} \\
\left(2(1-t)+2 \varphi\left(x_{0}, z, \nu\right)-1, x_{0}-f_{0}\left(x_{0}, z, \nu\right), z\right), & \frac{1}{2} \leq t \leq 1,
\end{aligned}\right.
$$

after the approximation of $\left(f_{0}, f_{\infty}\right)$ on $E_{n}$ by $\left(f_{n, 0}, f_{n}\right)$ and $\left(x_{0}, z\right)$ replaced by $\left(x_{0, n}, z_{n}\right)$ and $z_{i}=\xi_{i}^{m_{i} / n_{0}}, n_{0}$ a common divisor of $m_{1}, \ldots, m_{n}$. If $\operatorname{deg}\left(x-f(x, \nu), \Omega^{S^{1}}\right)=0$, then the map $g$ can be replaced by $x-f(x, \nu)$.

We would like to point out that this definition is independent of $n$ and $n_{0}$ since

$$
\begin{aligned}
\operatorname{deg}_{K, n_{0}}\left(g_{n}\right) & =n_{0}^{-n+1} \operatorname{deg}_{K, 1}\left(g_{n}\right)=\operatorname{deg}_{K, m_{0}}\left(g_{m}\right) \\
& =m_{0}^{-m+1} \prod_{n+1}^{m} m_{i} \operatorname{deg}_{K, 1}\left(g_{n}\right)
\end{aligned}
$$

This degree has of course all the properties of any topological degree; in fact, it comes from $\operatorname{deg}_{S^{1}}(x-f(x, \nu), \Omega)$.

Remark 4.12. If $x-f(x, \nu) \neq 0$ on $\Omega^{S^{1}}$, for example, if $\Omega^{S^{1}}=\varnothing$, then the first component of the normalized $S^{1}$-degree is 0 and the second component is $\left(\prod_{1}^{n} m_{i}\right)^{-1} \operatorname{deg}_{K}\left(2 t+2 \varphi(Y)-1, x_{0}-f_{0}(Y), Z_{1}^{m_{1}}-f_{n, 1}(Y), \ldots, Z_{n}^{m_{n}}-f_{n, n}(Y)\right)$ where $Y=\left(x_{0}, Z_{1}^{m_{1}}, \ldots, Z_{n}^{m_{n}}, \nu\right)$.

The generalized Fuller degree and the normalized index. If we restrict our attention to maps (and homotopies) with the property that they have no stationary solutions on $\Omega$, then we shall call the second component of the normalized $S^{1}$-degree the generalized Fuller degree of $x-f(x, \nu)$ in $\Omega$. This degree will have all the properties of a usual degree.

We shall now define the normalized index of an isolated orbit in the case of a nonstationary orbit. Suppose that $\left(x^{0}, \nu^{0}\right)$ is a solution of

$$
x-f(x, \nu)=0
$$

and that $x-f(x, \nu) \neq 0$ on an invariant neighborhood of $\left(x^{0}, \nu^{0}\right)$ for any $(x, \nu) \neq\left(e^{i \varphi} x^{0}, \nu^{0}\right)$. Then one may define the normalized index of the orbit as the normalized degree for any small neighborhood of the orbit. Now if $x^{0}$ 
is not a stationary point of (4.7) then we can write $x^{0}=\left(x_{0}^{0}, z^{0}\right)$, where $x_{0}^{0}$ is the invariant part of $x^{0}$ and $z^{0} \neq 0$.

For instance, we may assume that the first component $z_{1}^{0}$ of $z^{0}$ is nonzero. Moreover, by the action of the group, $z_{1}^{0}>0$. We may choose the invariant neighborhood of the orbit in such a way that $z_{1} \neq 0$ on it. Thus,

$$
\varphi\left(x_{0}, 0, z_{2}, \ldots, z_{n}, \nu\right)=1 \text {. }
$$

This implies that $p_{*}\left([x-f(x, \nu)]_{S^{1}}\right)=\operatorname{deg}\left(x-f(x, \nu), \Omega^{S^{1}}\right)=0$ and $(2 t+$ $2 \varphi(x, \nu)-1, x-f(x, \nu)) \neq 0$ if the $z_{1}$-component of $x$ is zero.

Hence, the rational component of the normalized index is just the Brouwer degree, divided by $\Pi_{1}^{n} m_{i}$, of the map

$$
\begin{aligned}
& \left(2 t+2 \varphi\left(x_{0}, Z_{1}^{m_{1}}, \ldots, Z_{n}^{m_{n}}, \nu\right)-1, x_{0}-f_{n, 0}\left(x_{0}, Z_{1}^{m_{1}}, \ldots, Z_{n}^{m_{n}}, \nu\right),\right. \\
& \left.\quad Z_{1}^{m_{1}}-f_{n, 1}\left(x_{0}, Z_{1}^{m_{1}}, \ldots, Z_{n}^{m_{n}}, \nu\right), \ldots, Z_{n}^{m_{n}}-f_{n, n}\left(x_{0}, Z_{n}^{m_{n}}, \ldots, Z_{n}^{m_{n}}, \nu\right)\right)
\end{aligned}
$$

with respect to the set

$$
C=\partial\left\{t \in[0,1],\left\|x_{0}\right\| \leq R_{0},\|Z\| \leq R, 0 \leq Z_{1} \leq R,|\nu| \leq R_{0}\right\} .
$$

Now the map $\left(t, x_{0}, Z_{1}, \ldots, Z_{n}, \nu\right) \mapsto\left(t, x_{0}, Z_{1}^{m_{1}}, \ldots, Z_{n}^{m_{n}}, \nu\right)$ from $C$ into the set

$$
C^{\prime}=\partial\left\{t \in[0,1],\left\|x_{0}\right\| \leq R_{0},\|z\| \leq R^{\prime}, 0 \leq z_{1} \leq R^{\prime},|\nu| \leq R_{0}\right\}
$$

has degree $\prod_{2}^{n} m_{i}$. Thus, by the composition law, the normalized index is the Brouwer degree, divided by $m_{1}$, of the map

$$
\begin{aligned}
& \left(2 t+2 \varphi\left(x_{0}, z_{1}, \ldots, z_{n}, \nu\right)-1, x_{0}-f_{n, 0}\left(x_{0}, z_{1}, \ldots, z_{n}, \nu\right),\right. \\
& \left.\quad z_{1}-f_{n, 1}\left(x_{0}, z_{1}, \ldots, z_{n}, \nu\right), \ldots, z_{n}-f_{n, n}\left(x_{0}, z_{1}, \ldots, z_{n}, \nu\right)\right)
\end{aligned}
$$

with respect to the set $C^{\prime}$. To compute the degree of the above map we may proceed by computing the Leray-Schauder degree of the map

$$
\begin{gathered}
\left(2 t+2 \varphi\left(x_{0}, z, \nu\right)-1, x_{0}-f_{0}\left(x_{0}, z, \nu\right), z-f_{\infty}\left(x_{0}, z, \nu\right)\right) \\
=(2 t+2 \varphi(x, \nu)-1, x-f(x, \nu))
\end{gathered}
$$

with respect to the set

$$
\partial\left\{t \in[0,1],\left\|x_{0}\right\| \leq R_{0},\|z\| \leq R^{\prime}, 0 \leq z_{1} \leq R^{\prime},|\nu| \leq R_{0}\right\},
$$

where $z=\left(z_{1}, \ldots, z_{n}\right)$.

By the additivity property of the degree and the construction of $\varphi$, the LeraySchauder degree of the above map is the sum of the indices (with $z_{1} \in \mathbf{R}^{+}$) of $x-f(x, \nu)$ at the points $\left(x^{j}, \nu\right)$ in $\Omega$ with $z_{1}^{j}>0$, that is, points on the orbit of $x^{0}$ with $z_{1}^{j}>0: x^{j}=e^{i \varphi} x^{0}$, or else $\nu=\nu^{0}, x_{0}^{j}=x_{0}^{0}, z_{n}^{j}=e^{i m_{n} \varphi} z_{n}^{0}$.

But $z_{1}^{j}=e^{i m_{1} \varphi} z_{1}^{0}>0$ implies that $\varphi$ is a multiple of $2 \pi / m_{1} \quad(\varphi=0$ corresponds to $x^{0}$ ). 
However, two of the $x^{j}$ (for two multiples $0 \leq l<k<m_{1}$ of $2 \pi / m_{1}$ ) will coincide if $z_{n}^{0} e^{i m_{n} 2 k \pi / m_{1}}=z_{n}^{0} e^{i m_{n} 2 l \pi / m_{1}}$, that is, either $z_{n}^{0}=0$ or $0<k-l=$ $h_{n} m_{1} / m_{n}$ for some positive integer $h_{n}<m_{n}$. Since $k-l<m_{1}$, then $m_{1}$ and $m_{n}$ have a common factor: $m_{1}=a(k-l), m_{n}=a h_{n}$, and $k-l$ is a multiple of $m_{1} / n_{0}$, if $n_{0}$ is the largest common factor of $m_{1}, \ldots, m_{n}, \ldots$ for all $n$ 's such that $z_{n}^{0} \neq 0$. Thus there are only $m_{1} / n_{0}$ different points $x^{j} \in \Omega$, with $z_{1}^{j}>0$, where $n_{0}$ is the order of the isotropy subgroup of $x^{0}$, $x^{j}=e^{i 2 \pi j n_{0} / m_{1}} x^{0}, j=0, \ldots, m_{1} / n_{0}-1$.

For any neighborhood of $\left(x^{j}, \nu^{0}\right)$, intersected with the space $z_{1} \in \mathbf{R}^{+}$, one may perform the homotopy (of the form Id-compact)

$$
x-e^{i 2 \pi j\left(n_{0} / m_{1}\right) \tau} f\left(e^{-i 2 \pi j\left(n_{0} / m_{1}\right) \tau} x, \nu\right), \quad \tau \in[0,1]
$$

(which is equal to $x-f(x, \nu)$ by the equivariance and therefore admissible).

Now, for $\tau=1$ the map $(x, \nu) \mapsto\left(e^{-i 2 \pi j\left(n_{0} / m_{1}\right)} x, \nu\right)$ is an isomorphism, from the neighborhood of $\left(x^{j}, \nu^{0}\right)$ onto the neighborhood of $\left(x^{0}, \nu^{0}\right)$, with degree 1. Thus the local indices are equal at each $\left(x^{j}, \nu^{0}\right)$. (Since the isomorphism is not of the form Id-compact, one has to consider first its finitedimensional approximations; then the above isomorphism has degree 1 , since its real determinant is 1 and the indices on $E_{n}$ are equal and hence, by the suspension theorem, the Leray-Schauder indices will also be equal).

We will summarize what we have just proved in the following.

Proposition 4.13. If $\left(e^{i \varphi} x^{0}, \nu^{0}\right)$, with $z_{1}^{0}>0$, denotes an isolated solution (nonstationary) of $x-f(x, \nu)$, then its normalized $S^{1}$-index is

$$
\left(0,\left(1 / n_{0}\right) \operatorname{Index}\left(x-f(x, \nu), x^{0},\left(\mathbf{R}^{N+1} \times E\right) \cap\left\{z_{1} \in \mathbf{R}^{+}\right\}\right)\right),
$$

where $n_{0}$ is the order of the isotropy subgroup of the orbit and Index $(\cdot, \cdot, \cdot)$ stands for the Leray-Schauder topological index at $\left(x^{0}, \nu^{0}\right)$ of the map $x-f(x, \nu)$, where one takes the Poincaré section $\operatorname{Im} z_{1}=0$.

Observe that, due to its construction, the normalized $S^{1}$-index is independent of the Poincaré section chosen.

We would like to note that one could have defined the normalized $S^{1}$-index using the methods given in [I.M.V]. Namely, given a tubular neighborhood $\Omega$ of the orbit, one obtains $\left(\prod_{J} m_{i}\right) / n_{0}$ tubular neighborhoods in the space $\left(x_{0}, Z_{1}, \ldots, Z_{n}, \nu\right)$, where $J$ denotes the subset of $j$ 's such that $Z_{j}^{0} \neq 0$. (One counts the inverse images and then identifies those which are identified under the isotropy subgroup of the orbit. Note that for the approximation $f_{n}$ of $f$ one may have more than one orbit in $\Omega_{n}$, but at the level of the neighborhoods the argument goes through.) Then the degree of the map

$$
\begin{aligned}
& \left(2 t+2 \varphi\left(x_{0}, Z_{1}^{m_{1}}, \ldots, Z_{n}^{m_{n}}, \nu\right)-1, x_{0}-f_{n, 0}\left(x_{0}, Z_{1}^{m_{1}}, \ldots, Z_{n}^{m_{n}}, \nu\right),\right. \\
& \left.\quad Z_{1}^{m_{1}}-f_{n, 1}\left(x_{0}, Z_{1}^{m_{1}}, \ldots, Z_{n}^{m_{n}}, \nu\right), \ldots, Z_{n}^{m_{n}}-f_{n, n}\left(x_{0}, Z_{1}^{m_{1}}, \ldots, Z_{n}^{m_{n}}, \nu\right)\right)
\end{aligned}
$$


is the sum of the indices of the map

$$
\begin{aligned}
\left(x_{0}-f_{n, 0}\left(x_{0}, Z_{1}^{m_{1}}, \ldots, Z_{n}^{m_{n}}, \nu\right), Z_{1}^{m_{1}}-f_{n, 1}\left(x_{0}, Z_{1}^{m_{1}}, \ldots, Z_{n}^{m_{n}}, \nu\right), \ldots,\right. \\
\left.Z_{n}^{m_{n}}-f_{n, n}\left(x_{0}, Z_{1}^{m_{1}}, \ldots, Z_{n}^{m_{n}}, \nu\right)\right)
\end{aligned}
$$

at each of these points. By the composition property of the degree, we obtain then that it is the degree of the above map at $x_{n}^{0}$ (on the space $Z_{1}$ real) multiplied by the index of the map

$$
\left(x_{0}, Z_{1}, \ldots, Z_{n}, \nu\right) \mapsto e^{-i 2 \pi j\left(n_{0} / m_{1}\right)}\left(x_{0}, Z_{1}^{m_{1}}, \ldots, Z_{n}^{m_{n}}, \nu\right), \quad Z_{1} \in \mathbf{R}^{+},
$$

at the point $\left(x_{0}^{0}, Z_{1}^{0}, \ldots, Z_{n}^{0}, \nu^{0}\right)$.

If $Z_{j}^{0}$ is nonzero, then the contribution of $Z_{j}^{m_{j}}$ is 1 , while if $Z_{j}^{0}=0$ the contribution will be $m_{j}$.

Thus the index at each $\left(\Pi_{J} m_{i}\right) / n_{0}$ points is

$$
\prod_{J^{c}} m_{i} \operatorname{Index}\left(x-f_{n}(x, \nu), x^{0}, E_{n} \cap\left\{z_{1} \in \mathbf{R}^{+}\right\}\right) \text {. }
$$

Note that one may consider a small neighborhood of $\left(x^{0}, \nu^{0}\right)$ in $\mathbf{R}^{N} \times E \times \mathbf{R}$ and the normalized $S^{1}$-index of the orbit will be the index, divided by $n_{0}$, of the map $\left(x-f(x, \nu),-\operatorname{Im} z_{1}\right)$ at $\left(x^{0}, \nu^{0}\right)$ (by the product theorem, the map $\left(x_{0}, x_{1}+i y_{1}, z_{2}, \ldots, z_{n}, \nu\right) \mapsto\left(x_{0}, x_{1}, z_{2}, \ldots, z_{n}, \nu, y_{1}\right)$ has degree -1$)$.

Computation of the normalized index. We suppose now that the map $f$ is of class $C^{1}$ and that $x^{0}$ belongs to the domain of the infinitesimal generator $A$ of the group $x \mapsto e^{i \varphi} x$; that is, $d\left(e^{i \varphi} x^{0}\right) / d \varphi$ belongs to $\mathbf{R}^{N} \times E$. This requires some smoothness on the orbit $e^{i \varphi} x^{0}$. For example, if $E$ has the property that its norm is equivalent to the norm $\|x\|^{2}=\left\|x_{0}\right\|^{2}+\sum_{1}^{+\infty}\left|z_{n}\right|^{2}$, $x=\left(x_{0}, z_{1}, z_{2}, \ldots\right)$, then

$$
A x^{0}=\left(0, i m_{1} z_{1}, \ldots, i m_{n} z_{n}, \ldots\right) \in E
$$

if $\sum_{1}^{+\infty} m_{n}^{2}\left|z_{n}\right|^{2}<+\infty$. The operator $A$, considered as a real operator, has the form $A\left(x_{0}, x_{1}, y_{1}, \ldots\right)=\left(0,-m_{1} y_{1}, m_{1} x_{1}, \ldots\right)$.

Note that if $x^{0}$ belongs to the domain of $A$, so do $e^{i \varphi} x^{0}$ and $A e^{i \varphi} x^{0}=$ $e^{i \varphi} A x^{0}$. Then, since $e^{i \varphi} x^{0}-f\left(e^{i \varphi} x^{0}, \nu^{0}\right)=0$, one has that $A x^{0}-f_{x}\left(x^{0}, \nu^{0}\right) A x^{0}$ $=0$; that is, $A x^{0}$ belongs to the real eigenspace of the (real) compact operator $f_{x}\left(x^{0}, \nu^{0}\right)$, corresponding to the eigenvalue 1 .

On the space $\mathbf{R}^{N} \times E \times \mathbf{R}$, consider the compact operator

$$
K(x, \mu)=\left(f_{x}\left(x^{0}, \nu^{0}\right) x+f_{\nu}\left(x^{0}, \nu^{0}\right) \mu, \mu+\operatorname{Im} z_{1}\right) .
$$

We now study the kernel of $I-K$.

If $(I-K)(x, \mu)=0$, then $f_{\nu}\left(x^{0}, \nu^{0}\right) \mu \in \operatorname{Range}\left(I-f_{x}\left(x^{0}, \nu^{0}\right)\right)$. If this is the case, then there is a unique $\bar{x}$ (in a fixed complement of $\operatorname{ker}\left(I-f_{x}\left(x^{0}, \nu^{0}\right)\right)$ ) such that $\left(I-f_{x}\left(x^{0}, \nu^{0}\right)\right) \bar{x}=f_{\nu}\left(x^{0}, \nu^{0}\right)$. Thus, $\mu \bar{x}$ will be a solution of $x-$ 
$f_{x}\left(x^{0}, \nu^{0}\right) x-f_{\nu}\left(x^{0}, \nu^{0}\right) \mu=0$ as well as $\mu \bar{x}+\alpha \underline{x}$, for any $\underline{x} \in$ $\operatorname{ker}\left(I-f_{x}\left(x^{0}, \nu^{0}\right)\right)$. On the other hand, since $\left(A x^{0}\right)_{1}=i m_{1} z_{1}^{0}$ is purely imaginary, given a basis $\left\{A x^{0}, x^{1}, \ldots, x^{n}\right\}$ of $\operatorname{ker}\left(I-f_{x}\left(x^{0}, \nu^{0}\right)\right)$, then the set of vectors $\left\{A x^{0}, x^{1}+\alpha_{1} A x^{0}, \ldots, x^{n}+\alpha_{n} A x^{0}\right\}$, with $\alpha_{i}=-\operatorname{Im} z_{1}^{i} / m_{1} z_{1}^{0}$, is also a basis. Thus one may assume that $x^{1}, \ldots, x^{n}$ have $\operatorname{Im} z_{1}^{i}=0$. The condition $\operatorname{Im}(\mu \bar{x}+\alpha \underline{x})_{1}=0$ means $\mu \operatorname{Im} \bar{z}_{1}+\alpha \operatorname{Im} \underline{z}_{1}=0$. Hence, if $\operatorname{Im} \bar{z}_{1} \neq 0$, we may satisfy this condition with $\alpha_{0}(\mu)=-\mu \operatorname{Im} \bar{z}_{1} / m_{1} z_{1}^{0}$ for $\underline{x}=A x^{0}$, and for $\mu=0$ for $x^{1}, \ldots, x^{n}$. Thus $\operatorname{ker}(I-K)$ has the same dimension as $\operatorname{ker}\left(I-f_{x}\left(x^{0}, \nu^{0}\right)\right)$ and it is generated by the vectors $\left(\bar{x}+\alpha_{0}(1) A x^{0}, 1\right),\left(x^{1}, 0\right), \ldots,\left(x^{n}, 0\right)$ (since $\mu \bar{x}+\underline{x}=\mu \bar{x}+\lambda_{0} A x^{0}+\sum \lambda_{i} x^{i}$, and $\left.\lambda_{0}=\alpha_{0}(\mu)\right)$. If $\operatorname{Im} \bar{z}_{1}=0$ (for example, if $f_{\nu}\left(x^{0}, \nu^{0}\right)=0$ then $\left.\bar{x}=0\right)$, the vectors $(\bar{x}, 1),\left(x^{1}, 0\right), \ldots,\left(x^{n}, 0\right)$ are generators. In both cases the operator $I-K$ is not invertible and

$$
\operatorname{dim} \operatorname{ker}(I-K)=\operatorname{dim} \operatorname{ker}\left(I-f_{x}\left(x^{0}, \nu^{0}\right)\right) .
$$

If $f_{\nu}\left(x^{0}, \nu^{0}\right) \mu \notin \operatorname{Range}\left(I-f_{x}\left(x^{0}, \nu^{0}\right)\right)$, then $\mu=0$ and the kernel of $I-K$ is generated by $\left(x^{1}, 0\right), \ldots,\left(x^{n}, 0\right)$, with one dimension less than $\operatorname{ker}\left(I-f_{x}\left(x^{0}, \nu^{0}\right)\right)$.

Definition 4.14. We shall say that the orbit $\left(e^{i \varphi} x^{0}, \nu^{0}\right)$ is hyperbolic if $I-K$ is invertible, i.e., if

(a) $f_{\nu}\left(x^{0}, \nu^{0}\right) \notin \operatorname{Range}\left(I-f_{x}\left(x^{0}, \nu^{0}\right)\right)$;

(b) $\operatorname{dim} \operatorname{ker}\left(I-f_{x}\left(x^{0}, \nu^{0}\right)\right)=1$, i.e., $A x^{0}$ is the generator.

We shall say that the orbit is simply hyperbolic if it is hyperbolic and the algebraic multiplicity of 1 , as eigenvalue of $f_{x}\left(x^{0}, \nu^{0}\right)$, is 1 .

Note that these definitions are independent of the representative $x^{0}$ on the orbit. Indeed, since $\left(I-f_{x}\left(e^{i \varphi} x^{0}, \nu^{0}\right)\right) x=e^{i \varphi}\left(I-f_{x}\left(x^{0}, \nu^{0}\right)\right) e^{-i \varphi} x$, we have that $\operatorname{ker}\left(I-f_{x}\left(e^{i \varphi} x^{0}, \nu^{0}\right)\right)$ is generated by $A e^{i \varphi} x^{0}$ and $f_{\nu}\left(e^{i \varphi} x^{0}, \nu^{0}\right)$ $=e^{i \varphi} f_{\nu}\left(x^{0}, \nu^{0}\right)$, so that $f_{\nu}\left(e^{i \varphi} x^{0}, \nu^{0}\right)=\left(I-f_{x}\left(e^{i \varphi} x^{0}, \nu^{0}\right)\right) x$ if and only if $f_{\nu}\left(x^{0}, \nu^{0}\right)=\left(I-f_{x}\left(x^{0}, \nu^{0}\right)\right) e^{-i \varphi} x$.

Hence, if $\left(e^{i \varphi} x^{0}, \nu^{0}\right)$ is hyperbolic, the Leray-Schauder index of the map $\left(x-f(x, \nu),-\operatorname{Im} z_{1}\right)$ at $\left(x^{0}, \nu^{0}\right)$ is the index of the operator $I-K$ at $(0,0)$, which is the index of $I-\lambda K$ for $\lambda$ close to 1 , that is, $(-1)^{\sum \alpha_{i}}$, where $\alpha_{i}$ is the algebraic multiplicity of the characteristic eigenvalue $\lambda_{i}<1$ and the sum is over all such characteristic eigenvalues.

Case of a simply hyperbolic orbit. If the orbit is simply hyperbolic, then

$$
\mathbf{R}^{N} \times E=\operatorname{ker}\left(I-f_{x}\right) \oplus \operatorname{Range}\left(I-f_{x}\right)
$$

so that $f_{\nu}\left(x^{0}, \nu^{0}\right)=\alpha A x^{0} \oplus w$ where $w \in \operatorname{Range}\left(I-f_{x}\right)$ and $\alpha \neq 0$. Now consider the deformation,

$$
\left(\left(I-f_{x}\right) x-\alpha \mu A x^{0}-\tau \mu w,-\operatorname{Im} z_{1}\right), \quad \tau \in[0,1] .
$$


This is an admissible homotopy since the only zero is for $\mu=0 \quad\left(\alpha A x^{0} \notin\right.$ $\left.\operatorname{Range}\left(I-f_{x}\right)\right), x=A x^{0}$, and $\operatorname{Im} x=m_{1} z_{1}^{0} \neq 0$. Thus one may replace $f_{\nu}$ by $\alpha A x^{0}$ in the definition of $K$. Also for $\lambda<1$, so close to 1 that $I-\lambda K$ and $I-\lambda f_{x}$ are invertible (the operators $K$ and $f_{x}$ are both compact, hence with discrete spectrum, so since $\lambda=1$ is in the spectrum of $f_{x}$, then $\lambda^{-1}$ cannot be an eigenvalue if $\lambda$ is close to 1 and $\lambda \neq 1$ ), one may write

$$
\begin{aligned}
(I-\lambda K)(x, \mu) & =\left(\left(I-\lambda f_{x}\right) x-\alpha \mu A x^{0}, \mu(1-\lambda)-\operatorname{Im} z_{1}\right) \\
& =\left(\left(I-f_{x}\right)\left(x-\mu k A x^{0}\right), \mu \alpha(\lambda)-\operatorname{Im}\left(z_{1}-\mu k i m_{1} z_{1}^{0}\right)\right)
\end{aligned}
$$

where $\alpha(\lambda)=(1-\lambda)-\alpha m_{1} z_{1}^{0} /(1-\lambda)$ and $k=\alpha /(1-\lambda)$. Note that $\alpha(\lambda) \neq 0$ for $\lambda<1$ and close to 1 and $\alpha(\lambda)$ has the sign of $-\alpha$. Replacing $k$ by $k \tau$ (the only zero will be $\left(\mu=0, x=k \tau \mu A x^{0}=0\right)$ ), one has to look at the index of $\left(\left(I-\lambda f_{x}\right) x, \mu \alpha(\lambda)\right)$, which is (deforming $\alpha(\lambda)$ to $-\operatorname{sign} \alpha$ ) just $-\operatorname{sign} \alpha \operatorname{Index}\left(I-\lambda f_{x}\right)$.

Case of a hyperbolic orbit. If the orbit is hyperbolic, then the above argument applies after writing $\mathbf{R}^{N} \times E=E^{*} \oplus \operatorname{Range}\left(I-f_{x}\right)$, where $E^{*}$ is some complement of $\operatorname{Range}\left(I-f_{x}\right)$. In this case we have that $f_{\nu}\left(x^{0}, \nu^{0}\right)=y^{*}+\left(I-f_{x}\right) y$, with $y^{*} \neq 0$, so that

$$
(I-K)(x, \mu)=\left(\left(I-f_{x}\right)(x+\mu y)-\mu y^{*},-\operatorname{Im} z_{1}\right) .
$$

Now replace $y$ by $\tau y$ and consider the index of $\left(\left(I-f_{x}\right) x-\mu y^{*},-\operatorname{Im} z_{1}\right)$ or else, for $\lambda<1, \lambda$ close to 1 , the index of $\left(\left(I-\lambda f_{x}\right) x-\lambda \mu y^{*},(1-\lambda) \mu-\lambda \operatorname{Im} z_{1}\right)$. Fix $\lambda<1$, so close to 1 that $I-\lambda f_{x}$ is invertible. Then $I-\lambda K$ will also be invertible with the same index. Then $y^{*} \in \operatorname{Range}\left(I-\lambda f_{x}\right)$, so that $y^{*}=$ $\left(I-\lambda f_{x}\right) z(\lambda)$ (in the previous case $\left.y^{*}=\alpha A x^{0}, z(\lambda)=k A x^{0}\right)$ and $(I-\lambda K)(x, \mu)=\left(\left(I-\lambda f_{x}\right)(x-\mu \lambda z(\lambda)), \mu\left(1-\lambda-\operatorname{Im} \lambda z_{1}(\lambda)\right)-\operatorname{Im}\left(z_{1}-\mu \lambda z_{1}(\lambda)\right)\right)$. Now, since $I-\lambda K$ is invertible, the only zero is at $(x=0, \mu=0)$. But then

$$
x-\mu \lambda z(\lambda)=0, \quad \mu\left(1-\lambda-\operatorname{Im} \lambda z_{1}(\lambda)\right)=0 .
$$

If we set $\alpha(\lambda)=1-\lambda-\lambda \operatorname{Im} z_{1}(\lambda)$, then $\alpha(\lambda) \neq 0$ (if not, the vector $(\mu \lambda z(\lambda), \mu$ ) would be in $\operatorname{ker}(I-\lambda K))$. Deform $x-\mu \lambda z(\lambda)$ to $x$, as well as $z_{1}-\mu \lambda z_{1}(\lambda)$ to $z_{1}$ and then $\alpha(\lambda)$ to $\operatorname{sign} \alpha(\lambda)$. Thus,

$$
\operatorname{Index}(I-\lambda K)=\operatorname{sign} \alpha(\lambda) \operatorname{Index}\left(I-\lambda f_{x}\right) .
$$

What we have just proved is given in the following.

Proposition 4.15. The normalized $S^{1}$-index of an isolated hyperbolic orbit is $\left(0, \operatorname{sign} \alpha(\lambda)(-1)^{\sum n_{i}} / n_{0}\right)$, where $n_{i}$ is the algebraic multiplicity of $\lambda_{i}^{-1}$ as an eigenvalue of $f_{x}, 0<\lambda_{i}<1, n_{0}$ is the order of the isotropy subgroup of the orbit, $\lambda$ is fixed, close to 1 , and $\lambda<1$. If the orbit is simply hyperbolic, with $f_{\nu}\left(x^{0}, \nu^{0}\right)=\alpha A x^{0} \oplus w$, then $\operatorname{sign} \alpha(\lambda)=-\operatorname{sign} \alpha$.

4.5. Applications. It is our aim now to show how the $S^{1}$-degree is related to Fuller's and Dancer's degrees. 
Autonomous differential equations: Fuller's degree. In [F], Fuller defined an index for sets of periodic orbits of the autonomous differential equation

$$
d \tilde{x} / d t=g(\tilde{x})
$$

where $g$ is a $C^{1}$-vector field over a smooth differential manifold $M$ in $\mathbf{R}^{M}$.

We shall restrict our discussion to the case $M=\mathbf{R}^{M}$. By the regularity of the vector field $g$, the autonomous differential equation (4.8) has, for each initial point $\tilde{x} \in \mathbf{R}^{M}$, a unique solution $\tilde{Y}(\tilde{x}, t)$. Note that $\tilde{Y}(\tilde{x}, 0)=\tilde{x}$ and that $\widetilde{Y}(\tilde{x}, t+s)=\tilde{Y}(\tilde{Y}(\tilde{x}, t), s)$; thus the translation on $t$ gives an action on $\mathbf{R}^{M}$.

Our study will not be in the geometrical space $\mathbf{R}^{M}$ as done by Fuller, but in the space of $2 \pi$-periodic functions, where the group action is given explicitly in terms of Fourier series. For this, by letting $\tau=\nu t$, the differential equation (4.8) becomes

$$
\nu \dot{y}(\tau)=g(y(\tau)), \quad \dot{y}=d y / d \tau .
$$

Thus $2 \pi / \nu$-periodic solutions of (4.8) correspond to $2 \pi$-periodic solutions of (4.9) and vice versa.

We shall identify the real numbers $\bmod 2 \pi$ with $S^{1}$ and the spaces $L^{2}\left(S^{1}\right)$, $W^{1,2}\left(S^{1}\right)$ of real functions $y: S^{1} \rightarrow \mathbf{R}$ with scalar products

$$
\left(y_{1}, y_{2}\right)=\int_{0}^{2 \pi}\left(y_{1} y_{2}+\varepsilon \dot{y}_{1} \dot{y}_{2}\right) d \tau, \quad \varepsilon=0,1,
$$

are identified with the space of Fourier series $\sum_{-\infty}^{+\infty} y_{n} e^{i n \tau}, y_{-n}=\bar{y}_{n}$, such that $\sum_{-\infty}^{+\infty}\left(1+\varepsilon n^{2}\right)\left|y_{n}\right|^{2}<\infty, \varepsilon=0,1$, respectively.

Observe that the map $y \mapsto \nu \dot{y}-g(y)$ defines a $C^{1}$-Fredholm operator from $W^{1,2}\left(S^{1}\right)^{M}$ into $L^{2}\left(S^{1}\right)^{M}$. Let $\widetilde{K}: L^{2}\left(S^{1}\right)^{M} \rightarrow W^{1,2}\left(S^{1}\right)^{M}$ be the continuous operator defined by

$$
\widetilde{K}\left(y_{0}+\sum_{n \neq 0} y_{n} e^{i n \tau}\right)=y_{0}+\sum_{n \neq 0} y_{n} e^{i n \tau} / i n .
$$

Clearly, the operator $\widetilde{K}$ can be regarded as a compact operator from $L^{2}\left(S^{1}\right)^{M}$ into itself as well from $W^{1,2}\left(S^{1}\right)^{M}$ into itself. Notice that the differential equation (4.9) is equivalent to the integral equation

$$
\nu z-\widetilde{K} g\left(x_{0}, z\right)=0
$$

where $y(\tau)=x_{0}+z=\sum_{-\infty}^{+\infty} x_{n} e^{i n \tau}, x_{-n}=\bar{x}_{n}$.

Let $E=\left\{x=\left(x_{0}, x_{1}, x_{2}, \ldots\right): x_{0} \in \mathbf{R}^{M}, x_{n} \in \mathbf{C}^{M}, n \geq 1\right\}$ be the Hilbert space endowed with the norm $\|x\|^{2}=\left|x_{0}\right|^{2}+\sum_{n>0} n^{2}\left|x_{n}\right|^{2}$. Define on $E$ an $S^{1}$-action as follows:

$$
e^{i \varphi} x=\left(x_{0}, e^{i \varphi} x_{1}, \ldots, e^{i n \varphi} x_{n}, \ldots\right),
$$


which corresponds to the translation $y(\tau+\varphi)$. Therefore, (4.8) is equivalent to the system of an infinite number of equations

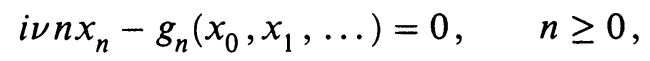

where

$g_{n}\left(x_{0}, x_{1}, \ldots\right):=g_{n}(y)=\frac{1}{2 \pi} \int_{0}^{2 \pi} g(y(\tau)) e^{-i n \tau} d \tau=\frac{\nu}{2 \pi} \int_{0}^{2 \pi / \nu} g(\tilde{x}(t)) e^{-i n \nu t} d t$.

Thus,

$$
g_{n}\left(e^{i \varphi} x\right)=\frac{1}{2 \pi} \int_{0}^{2 \pi} g(y(\tau+\varphi)) e^{-i n \tau} d \tau=e^{i n \varphi} g_{n}(x),
$$

that is, (4.11) gives a $S^{1}$-equivariant equation in $E$ of the form $x-f(x, \nu)=0$ with

$$
f(x, \nu)=(1 / \nu)\left(\nu x_{0}+g_{0}(x), g_{1}(x) / i, \ldots, g_{n}(x) / i n, \ldots\right) .
$$

To define the degree of the map $x-f(x, \nu)$ we have to make the following basic assumptions (see $[\mathrm{F}]$ ).

Let $\omega$ be an open bounded subset of $\mathbf{R}^{M} \times \mathbf{R}^{+}$such that the differential equation has no periodic solutions $\tilde{x}(t)$, with period $2 \pi / \nu$ (not necessarily minimal) such that $(\tilde{x}(t), \nu) \in \partial w$ for some $t$. This assumption ensures that if $(\tilde{x}, \nu) \in \omega$ and $\tilde{x}$ is a point on a periodic solution $\tilde{x}(t)$ with frequency $\nu$, then $(\tilde{x}(t), \nu) \in \omega$ for all $t$. Moreover, if $\tilde{x}$ is a stationary solution of (4.8), that is, $g(\tilde{x})=0$, then $(\tilde{x}, \nu)$ is also a solution for all $\nu$; thus, since $\omega$ is bounded $(\tilde{x}, \nu) \notin \omega$, that is, the set $\omega$ cannot contain stationary solutions. We shall also assume that $\nu \geq \delta>0$ in $\omega$.

If $(\tilde{x}, \nu) \in \omega$ with $\tilde{x}=\tilde{x}(t)$, for some periodic solution with frequency $\nu$, then $y(\tau)=\tilde{x}(t)$ is bounded and also $\dot{y}(\tau)=g(y(\tau)) / \nu$ will be bounded (uniformly on $\omega$ ). Hence the corresponding Fourier series will be bounded (uniformly for any periodic solution in $\omega$ ) by some constant $R$.

Let $\Omega=\left\{(x, \nu) \in E \times \mathbf{R}^{+}:\|x\| \leq R,(\tilde{x}(t), \nu) \in \omega\right\}$ where $\tilde{x}(t)=y(\tau)=$ $\sum_{-\infty}^{+\infty} x_{n} e^{i n \nu t}$ with $x_{-n}=\bar{x}_{n}$. Since any function in $W^{1,2}\left(S^{1}\right)$ is continuous, we have that if $(x, \nu)$ is close to $\left(x^{0}, \nu^{0}\right)$, then $\tilde{x}(t)$ will be close to $\tilde{x}^{0}(t)$ for all $t$. Then the set $\Omega$ is open. Clearly $\Omega$ is also invariant under the $S^{1}$-action (we leave $S^{1}$ to act trivially on $\mathbf{R}$ ). Finally, any periodic solution $(\tilde{x}(t), \nu) \in \omega$ of (4.8) will give exactly one solution $(x, \nu) \in \Omega$ of $x-f(x, \nu)=0$, and conversely. In particular, $x-f(x, \nu) \neq 0$ for all $(x, \nu) \in \partial \Omega$.

Now let $K: E \rightarrow E$ be the linear operator defined as

$$
K x=K\left(x_{0}, x_{1}, \ldots, x_{n}, \ldots\right)=\left(x_{0}, x_{1} / i, \ldots, x_{n} / \text { in }, \ldots\right) .
$$

Then we can write $f(x, \nu)=(1 / \nu)\left(\nu x_{0}+K g(x)\right)$. Clearly, the map $f$ is compact. Indeed, if $\left\{x^{n}\right\}$ is a bounded sequence in $E$, then $\left\{y^{n}(\tau)\right\}$ is bounded in $W^{1,2}\left(S^{1}\right)^{M}$, hence it has a converging subsequence in $C^{0}\left(S^{1}\right)^{M}$ (the embedding is compact) and $\left\{g\left(y^{n}(\tau)\right)\right\}$ will converge as well. Thus the normalized $S^{1}$-degree is well defined for the map $(x-f(x, \nu))$ on $\Omega$. 
Since stationary solutions $x_{0}$ of the differential equation (4.8), that is, $g\left(x_{0}\right)$ $=0$, correspond to invariant solutions of the equation $x-f(x, \nu)=0$ on $\Omega$, one has that $x_{0}-f_{0}\left(x_{0}, 0, \ldots, 0, \nu\right)=-(1 / \nu) g_{0}\left(x_{0}, 0, \ldots, 0\right)=-g\left(x_{0}\right) \neq 0$ in $\Omega$. Hence the degree of the invariant part of $x-f(x, \nu)$ is always zero.

We shall now compute the $S^{1}$-index of an isolated periodic solution. For this we first have to identify the derivatives of the map $f(x, \nu)$ and then apply Proposition 4.15.

If $\left(x^{0}, \nu^{0}\right)$ is a solution of $x-f(x, \nu)=0$, corresponding to the $2 \pi$-periodic solution $y^{0}(\tau)$, then

$$
f_{x}\left(x^{0}, \nu^{0}\right) x=x_{0}+\left(1 / \nu^{0}\right) K(B(\tau) y(\tau))
$$

Here $B(\tau)$ is the matrix $\left(g_{i, x_{j}}\left(y^{0}(\tau)\right)\right)_{i, j}$, where $g_{i}$ is the $i$ th component of the map $g$, and $B(\tau) y(\tau)=B * x$, the convolution of $B$ and $x$. Note that since $y(\tau)$ is continuous $B(\tau) y(\tau)$ is also continuous as well as $K(B y)$. Moreover,

$$
f_{\nu}\left(x^{0}, \nu^{0}\right)=-\frac{1}{\nu^{0^{2}}} K g\left(x^{0}\right)=-\frac{1}{\nu^{0}}\left(0, x_{1}^{0}, x_{2}^{0}, \ldots\right)=\frac{1}{\nu^{0}}\left(x_{0}^{0}-x^{0}\right) \text {. }
$$

In what follows we shall use simplified notation: $f_{x}$ and $f_{\nu}$ will stand for $f_{x}\left(x^{0}, \nu^{0}\right)$ and $f_{\nu}\left(x^{0}, \nu^{0}\right)$, respectively.

Let $A$ be the infinitesimal generator of the group $S^{1}$, that is, $A x=\left(0, i x_{1}\right.$, $\left.2 i x_{2}, \ldots\right)$. Since $g$ is of class $C^{1}$, then $A x^{0} \in E$, corresponding to $\dot{y}^{0}(\tau)$ $\left(\ddot{y}^{0}(\tau)=B(\tau) \dot{y}^{0}(\tau)\right)$, so that $A K x=x-x_{0}=K A x$. If $\left(I-\lambda f_{x}\right)(x)=z$, then $x-\lambda x_{0}-\lambda\left(1 / \nu^{0}\right) K(B y)=z$. In particular, $z_{0}=(1-\lambda) x_{0}-\left(1 / \nu^{0}\right) \lambda(B y)_{0}$ and if $z$ belongs to the domain of $A$, by applying $A$, we have that $A x-$ $\lambda\left(1 / \nu^{0}\right)(B y)+(1-\lambda) x_{0}=A z+z_{0}$, which corresponds in $W^{1,2}\left(S^{1}\right)^{M}$ to the integrodifferential equation (for $\lambda \neq 1$ )

$$
\dot{y}-\left(1 / \nu^{0}\right) \lambda B y+(1-\lambda) x_{0}=\dot{z}+z_{0} .
$$

If $\lambda=1$ and $z=0$, then any element in $\operatorname{ker}\left(I-f_{x}\right)$ will give a $2 \pi$-periodic solution of $\dot{y}-\left(1 / \nu^{0}\right) B y=0$, and conversely since $(B y)_{0}=\nu^{0}(\dot{y})_{0}=0$. Moreover, if $\lambda=1$ and $z=f_{\nu}=-\left(1 / \nu^{0}\right)\left(x^{0}-x_{0}^{0}\right)=-\left(1 / \nu^{0}\right) K A x^{0}$, then a solution of $\left(I-f_{x}\right) x=f_{\nu}$ will give a solution of $\dot{y}-\left(1 / \nu^{0}\right) B y=-\left(1 / \nu^{0}\right) \dot{y}^{0}$ with $y \in \operatorname{ker}\left(d / d t-\left(1 / \nu^{0}\right) B\right)^{2}$, where $d / d t-\left(1 / \nu^{0}\right) B$ is a Fredholm operator of index 0 from $W^{1,2}\left(S^{1}\right)^{M}$ into $L^{2}\left(S^{1}\right)^{M}$. Thus, to say that the orbit is hyperbolic (in the sense of Definition 4.14) means that $\operatorname{ker}\left(d / d t-\left(1 / \nu^{0}\right) B\right)^{\alpha}$ is one dimensional for all $\alpha \geq 1$; that is, $\dot{x}^{0}$ is a simple eigenvector of $d / d t-$ $\left(1 / \nu^{0}\right) B$. This is the usual definition of hyperbolicity (in this setting simple hyperbolicity and hyperbolicity coincide).

We shall thus assume that the periodic solution is hyperbolic. As in the last section, we shall denote by $z_{1}$ one of its nonzero 1-dimensional (complex) components, with its corresponding harmonics $m_{1}$. For the solution recall that $z_{1}^{0}$ is real and positive. 
To compute the $S^{1}$-index, instead of looking at the integrodifferential equation, we shall now recall the argument given in [I.M.V]. For a hyperbolic orbit, the operator $(I-K)$ considered in $\S 4.4$ has the form $\left(\left(I-f_{x}\right) x-\mu f_{\nu},-\operatorname{Im} z_{1}\right)$ and is invertible. Hence, the operator $\left(\left(I-f_{x}\right) x-\mu f_{\nu}+\lambda K x,-\operatorname{Im} z_{1}\right)$ is also invertible provided that $\lambda$ is small. We also have that the index of the orbit is the same (a small compact perturbation does not alter the index). However, $\left(I-f_{x}+\lambda K\right) x=0$ is equivalent to

$$
x-x_{0}-\left(1 / \nu^{0}\right) K(B y)+\lambda K x=0 .
$$

In particular, $\lambda x_{0}=\left(1 / \nu^{0}\right)(B y)_{0}$ and, applying $A$ to (4.12), we have that $A x-\left(1 / \nu^{0}\right)(B y)+\lambda x=0$, corresponding to $\dot{y}-\left(\left(1 / \nu^{0}\right) B-\lambda\right) y=0$. But the operator $d / d t-\left(\left(1 / \nu^{0}\right) B-\lambda\right)$ has, on $W^{1,2}\left(S^{1}\right)^{M}$, a kernel generated by $y(\tau)=e^{-\lambda \tau} \Phi(\tau) w$ with $w \in \operatorname{ker}\left(\Phi(2 \pi)-e^{2 \pi \lambda} I\right)$, where $\Phi(\tau)$ is the fundamental matrix for $\dot{y}-\left(1 / \nu^{0}\right) B y, \Phi(0)=I$, and $e^{2 \pi \lambda}$ are the Floquet multipliers of the $n_{0}$ th iterate of the return map. Furthermore, the algebraic multiplicity of $d / d t-\left(\left(1 / \nu^{0}\right) B-\lambda\right)$ is the algebraic multiplicity of $\Phi(2 \pi)-e^{2 \pi \lambda} I$ (for details we refer to [I.M.V]). Thus for $\lambda>0$ and small, the operator $I-f_{x}+\lambda K$ is invertible. Now, the perturbed operator can be written as

$$
\left(\left(I-f_{x}+\lambda K\right)\left(x+\frac{1}{\lambda \nu^{0}} \mu A x^{0}\right),-\operatorname{Im}\left(z_{1}+\frac{1}{\lambda \nu^{0}} \mu A z_{1}^{0}+\frac{1}{\lambda \nu^{0}} m_{1} \mu z_{1}^{0}\right)\right)
$$

because $f_{\nu}=-\left(1 / \nu^{0}\right) K A x^{0}$ and $\left(I-f_{x}\right) A x^{0}=0$. Since $I-f_{x}+\lambda K$ is invertible, the deformations $x+\left(1 / \lambda \nu^{0}\right) \tau \mu A x^{0}$ and $\tau\left(\operatorname{Im} x+\left(1 / \lambda \nu^{0}\right) \mu \tau A x^{0}\right)$ are admissible. Hence, the normalized $S^{1}$-index of the hyperbolic orbit is

$$
\operatorname{Index}\left(I-f_{x}+\lambda K\right) / n_{0},
$$

provided $\lambda>0$ and small.

Increasing $\lambda$, one will get a possible change of index at a point $\lambda_{0}$ such that $e^{2 \pi \lambda_{0}}$ is a Floquet multiplier. To see this we shall argue as follows.

Let $T=I-f_{x}+\lambda_{0} K$, let $P_{0}$ be the projection onto the constants, $P_{0} x=x_{0}$, and let $E_{0}$ be the space of Fourier coefficients with $L^{2}$-norm. Then

$$
K\left(A+P_{0}\right)=I_{E}, \quad\left(A+P_{0}\right) K=I_{E_{0}} .
$$

Set $\widetilde{T}=\left(A+P_{0}\right) T$. Then $T=K \widetilde{T}$ and the operators $T$ and $\widetilde{T}$ have the same kernel. In fact, if $z=T x$ then $A z+z_{0}=\widetilde{T} x$ and if $y=\widetilde{T} x$ then $K y=T x$. Moreover, if $z=T x$ and $T z=0$ then also $\widetilde{T} z=0$ but not necessarily $\widetilde{T}^{2} x=0$, since the operators $T$ and $K$ do not commute unless $B$ is constant. Hence the generalized kernels of $T$ and $\widetilde{T}$ do not coincide in general.

Moreover, the operator $\widetilde{T}$ coincides with the map $A x-\left(1 / \nu^{0}\right) B y+\lambda_{0} x$, corresponding to the differential equation $\dot{y}-\left(1 / \nu^{0}\right) B y+\lambda_{0} y$, with generalized kernel of multiplicity equal to the algebraic multiplicity of $e^{2 \pi \lambda_{0}}$ as a Floquet 
multiplier. Then $T-\left(\lambda_{0}-\lambda\right) K$ will give, after the composition with $A+P_{0}$, the operator $\widetilde{T}-\left(\lambda_{0}-\lambda\right) I_{E_{0}}$. Both operators have a generalized algebraic multiplicity (as Fredholm operators) defined as the sign of the determinant of the matrix given, for $T-\left(\lambda_{0}-\lambda\right) K$, by

$$
\left(\lambda_{0}-\lambda\right)\left(I_{E}-Q\right) K\left(I_{E}-R Q K\left(\lambda_{0}-\lambda\right)\right)^{-1} P=B(\lambda),
$$

where $P$ is a projection from $E$ onto $\operatorname{ker} T, Q$ a projection from $E$ onto Range $T$, and $R$ is a pseudo-inverse of $T$ defined by $T R Q=Q, R T(I-P)=$ $I-P$.

Likewise, we have for the operator $T-\left(\lambda_{0}-\lambda\right) I_{E_{0}}$ that

$$
\left(\lambda_{0}-\lambda\right)\left(I_{E_{0}}-\widetilde{Q}\right)\left(I_{E}-\widetilde{R} \widetilde{Q}\left(\lambda_{0}-\lambda\right)\right)^{-1} P=\widetilde{B}(\lambda)
$$

where $\widetilde{T} \widetilde{R} \widetilde{Q}=\widetilde{Q}$ and $\widetilde{R} \widetilde{T}(I-P)=I-P$.

Now, for a given $Q$, one may choose $\widetilde{Q}=\left(A+P_{0}\right) Q K, \widetilde{R}=R K$ and conversely, for a given $\widetilde{Q}$, one may take $Q=K \widetilde{Q}\left(A+P_{0}\right), R=\widetilde{R}\left(A+P_{0}\right)$ (clearly, $\widetilde{Q}$ and $Q$ are projections onto Range $\widetilde{T}$ and Range $T$ respectively and $\widetilde{R}, R$ have the right properties). Moreover, $I_{E}-\widetilde{R} \widetilde{Q}\left(\lambda_{0}-\lambda\right)=I_{E}-R Q K\left(\lambda-\lambda_{0}\right)$, $I_{E_{0}}-\widetilde{Q}=\left(A+P_{0}\right)\left(I_{E}-Q\right) K$. Thus,

$$
\widetilde{B}(\lambda)=\left(A+P_{0}\right) B(\lambda)
$$

Hence, there is a change in the sign of the determinant of $B(\lambda)$ if and only if there is a change of sign in the determinant of $\widetilde{B}(\lambda)$. This will be the case if and only if the algebraic multiplicity of 0 , as an eigenvalue of $\widetilde{T}$, is odd (see $\left.\left[\mathrm{I}_{0}\right]\right)$.

Thus at each Floquet multiplier one has a change of index of $I-f_{x}+\lambda K$ equal to $(-1)^{n}$, where $n$ is the algebraic multiplicity of the multiplier itself.

Now consider, for $\lambda>0$, the deformation

$$
x-\tau x_{0}+\tau\left(\lambda K x-\left(1 / \nu^{0}\right) K B y\right), \quad \tau \in[0,1],
$$

which corresponds to the differential equation

$$
\dot{y}+(1-\tau) x_{0}+\tau\left(\lambda y-\left(1 / \nu^{0}\right) B y\right), \quad \tau \in[0,1] .
$$

Applying $A+P_{0}$ to (4.13), one obtains $A x+(1-\tau) x_{0}+\tau\left(\lambda x-\left(1 / \nu^{0}\right) B y\right)$. Moreover, multiplying the differential equation (4.14) by $y^{\text {tr }}$ and taking $\lambda>$ $\left(2 / \nu^{0}\right)|B|$, where $|B|=\max _{\tau}\left|B_{i, j}(\tau)\right|$, one gets, after integrating on $[0,2 \pi]$, that

$$
(1-\tau)\left|x_{0}\right|^{2}+\tau\left(\lambda\|y\|^{2}-\frac{1}{\nu^{0}}(B y, y)\right) \geq(1-\tau)\left|x_{0}\right|^{2}+\tau\left(\lambda-\frac{2}{\nu^{0}}|B|\right)\|y\|^{2} .
$$

Hence, (4.14) is an admissible homotopy when $\lambda$ is sufficiently large. Clearly, for $\tau=0$ the index is 1 . Thus we have the following. 
Proposition 4.16. The normalized $S^{1}$-index of the hyperbolic orbit $\left(e^{i \varphi} x^{0}, \nu^{0}\right)$ is $(-1)^{\sum n_{i}} / n_{0}$, where $n_{i}$ is the algebraic multiplicity of the Floquet multiplier $e^{2 \pi \lambda_{i}}>1$.

Therefore the normalized $S^{1}$-degree with respect to $\Omega$ is minus the Fuller degree on $\omega$. The minus sign comes from the fact that when defining Fuller's degree one looks at periods $T=2 \pi / \nu$. The change from periods to frequencies has degree -1 .

Gradient maps: Dancer's degree. In [Da], Dancer considered fixed points of maps which are gradients of real-valued functions $g(x)$ with the property that $g\left(e^{i \varphi} x\right)=g(x)$, where $x$ is in a real Hilbert space with an isometric action of $S^{1}$ given by $e^{i \varphi}$. Then

$$
\left(D g\left(e^{i \varphi} x\right), e^{i \varphi} h\right)=(D g(x), h)=\left(e^{-i \varphi} D g\left(e^{i \varphi} x\right), h\right) \text {. }
$$

Thus the map $D g$ is $S^{1}$-equivariant; i.e., $D g\left(e^{i \varphi} x\right)=e^{i \varphi} D g(x)$. Note that each 2-dimensional representation of the group $S^{1}$ will give rise to a complex structure; thus we shall write $f_{j}:=g_{x_{j}}+i g_{y_{j}}$ for the representation $\left(x_{j}, y_{j}\right)$, identified with $x_{j}+i y_{j}$. If we let $f=\left(f_{0}, f_{1}, \ldots\right)$, then clearly $f\left(e^{i \varphi} x\right)=$ $e^{i \varphi} f(x)$.

Furthermore, by taking the derivative of $g\left(e^{i \varphi} x\right)-g(x)$ with respect to $\varphi$, we have that $\operatorname{Re}(A x, f(x))=0$ where $A=\left(0, i m_{1}, \ldots\right)$ is the infinitesimal generator of the group.

If the map $f$ is compact and defined on an invariant open bounded subset $\Omega$ of the Hilbert space and $f(x) \neq x$, for any $x \in \partial \Omega \cup \Omega^{S^{1}}$, then Dancer defined a $S^{1}$-degree by first approximating the map $f$ with finite-dimensional $S^{1}$-equivariant gradient maps $f_{n}$ and then approximating the $f_{n}$ 's with maps which have only a finite number of orbits. For each of these orbits, Dancer defined an index which, in our notation, can be written as

Index $\left(\left(x_{0}-f_{0}(x), \operatorname{Re}\left(z_{1}-f_{1}(x)\right), z_{2}-f_{2}(x), \ldots, z_{n}-f_{n}(x)\right) ; E \cap\left\{z_{1} \in \mathbf{R}^{+}\right\}\right) / n_{0}$, for an orbit with $z_{1}^{0}>0$ and the order of its isotropy subgroup equal to $n_{0}$.

Now consider the equation

$$
\nu A x+x-f(x)=0,
$$

where the map $f$ is assumed to be finite dimensional. By taking the inner product of (4.15) with $A x$, one has that $\nu\|A x\|^{2}=0$; that is, either $x=x_{0} \in$ $\Omega$ and $x_{0}-f\left(x_{0}\right)=0$, which is not possible for $x \in \Omega^{S^{1}}$, or $\nu=0$ and then one has a solution of $x-f(x)=0$. Thus $e^{i \varphi} x^{0}$ is an isolated solution of $x-f(x)=0$ if and only if $\left(e^{i \varphi} x^{0}, 0\right)$ is an isolated solution of (4.15). Moreover, for (4.15) one has a normalized $S^{1}$-index given by

$$
\begin{gathered}
\frac{1}{n_{0}} \operatorname{Index}\left(x_{0}-f_{0}(x), i \nu m_{1} z_{1}+z_{1}-f_{1}(x), i \nu m_{2} z_{2}+z_{2}-f_{2}(x), \ldots,-\operatorname{Im} z_{1}\right) \\
=\operatorname{Index}\left(\nu A x+x-f(x),-\operatorname{Im} z_{1}\right),
\end{gathered}
$$

where the index is computed at $\left(\left(x^{0}, z_{1}^{0}, z_{2}^{0}, \ldots\right), 0\right)$. 
Let $U$ be a small neighborhood of $\left(x^{0}, 0\right)$ such that equation $(4.15)$ has $\left(x^{0}, 0\right)$ as the only solution. Consider in $U$ the deformation

(4.16) $\left(\tau \nu A x+\nu i(1-\tau) e_{1}+x-f(x)-(1-\tau) i \operatorname{Im}\left(z_{1}-f_{1}(x)\right) e_{1},-\operatorname{Im} z_{1}\right)$,

where $e_{1}=(0,1,0, \ldots, 0)$. We shall now show that (4.16) is admissible. Indeed, if we have a zero of (4.16) then, taking the inner product of the first component with $A x$, we obtain that

$$
\tau \nu\|A x\|^{2}+\nu(1-\tau) m_{1} \bar{z}_{1}+(x-f(x), A x)-(1-\tau) \bar{z}_{1} \operatorname{Im}\left(z_{1}-f_{1}\right)=0 .
$$

But in $U, z_{1}=\bar{z}_{1}>0$; thus the $z_{1}$-component of the equation $(4.16)=0$ is

$$
i \nu m_{1} \tau z_{1}+i \nu(1-\tau)+z_{1}-\operatorname{Re} f_{1}-i \operatorname{Im} f_{1}+i(1-\tau) \operatorname{Im} f_{1}=0
$$

Hence

$$
\begin{gathered}
\tau \nu\|A x\|^{2}+\nu(1-\tau) m_{1} z_{1}+(1-\tau) z_{1} \operatorname{Im} f_{1}=0, \\
\tau \operatorname{Im} f_{1}=\left(m_{1} \tau z_{1}+1-\tau\right) \nu .
\end{gathered}
$$

If $\tau=0$, then $\nu=0, \operatorname{Im} f_{1}=0$, and hence $x-f(x)=0$, which is not possible on $\partial U$. On the other hand, if $\tau \neq 0$ then $\operatorname{Im} f_{1}$ has the same sign as $\nu$. But if $\nu=0$, then $\operatorname{Im} f_{1}=0, x-f(x)=0$ and if $\nu \neq 0$ then $\tau=1$, $A x=0, x-f(x)=0$, which is not possible since $z_{1} \neq 0$.

Hence, (4.16) gives an admissible deformation. In particular, for $\tau=0$, we have that

$$
\begin{aligned}
& \left(\text { vie }_{1}+x-f(x)-i \operatorname{Im}\left(z_{1}-f_{1}\right) e_{1},-\operatorname{Im} z_{1}\right) \\
& \quad=\left(x_{0}-f_{0}, \operatorname{Re}\left(z_{1}-f_{1}\right)+i \nu, z_{2}-f_{2}, \ldots,-\operatorname{Im} z_{1}\right) .
\end{aligned}
$$

Since the map $\left(\operatorname{Im} z_{1}, \nu\right) \mapsto\left(\nu,-\operatorname{Im} z_{1}\right)$ has degree 1 , we have the following.

\section{Proposition 4.17.}

$$
\begin{aligned}
& \frac{1}{n_{0}} \operatorname{Index}\left(x_{0}-f_{0}, \operatorname{Re}\left(z_{1}-f_{1}\right), z_{2}-f_{2}, \ldots, z_{n}-f_{n} ; E \cap\left\{z_{1} \in \mathbf{R}^{+}\right\}\right) \\
& \quad=\text { normalized } S^{1} \text {-index of the orbit. }
\end{aligned}
$$

That is, Dancer's degree and our degree coincide.

Note that if $f$ is a $C^{1}$-map (this is the case if $g$ is $C^{2}$ ), then $I-f_{x}\left(x^{0}\right)$ is the derivative of the map $\nu A x+x-f(x)$ with respect to $x$ at $\left(x^{0}, 0\right)$, and $A x^{0}$ is its derivative with respect to $\nu$. Moreover, $A x^{0}$ is in the kernel of $I-f_{x}\left(x^{0}\right)$. Thus, to say that $\left(x^{0}, 0\right)$ is a hyperbolic orbit means that $A x^{0}$ generates $\operatorname{ker}\left(I-f_{x}\left(x_{0}\right)\right)$ and, since $A x^{0} \notin \operatorname{Range}\left(I-f_{x}\left(x_{0}\right)\right), 1$ is a simple eigenvalue of $f_{x}\left(x^{0}\right)$ (thus simple hyperbolicity coincides with hyperbolicity). Note that $f_{x}\left(x_{0}\right)$, being the Hessian of $g$, is symmetric; thus hyperbolicity means simply that $\operatorname{dim} \operatorname{ker}\left(I-f_{x}\left(x_{0}\right)\right)=1$. 
Proposition 4.18. If 1 is a simple eigenvalue of $f_{x}\left(x_{0}\right)$ for the infinite-dimensional problem, then the normalized index is $(-1)^{\sum n_{i}} / n_{0}$, where $n_{0}$ is the order of the isotropy subgroup of $x^{0}$ and $\sum n_{i}$ is the number of eigenvalues of $f_{x}\left(x_{0}\right)$ greater than 1.

Indeed, it is enough to approximate the map $f$ by $f_{n}$ ( $A x$ is not a compact operator). For this map $\alpha=-1$ (see Proposition 4.15) and the result follows.

Periodic solutions of differential equations with fixed period. Consider the problem of finding $2 \pi$-periodic solutions of the equation

$$
\dot{y}=g(y, \nu), \quad y \in \mathbf{R}^{M}, \nu \in \mathbf{R},
$$

in some domain $\omega \subset \mathbf{R}^{M} \times \mathbf{R}$ such that there are no solutions on $\partial \omega$. Clearly, $g(x, \nu)=\nu^{-1} g(x)$ would be the special case of the situation considered by Fuller.

As before, this problem can be converted into a $S^{1}$-equivariant problem in $E=\left\{\left(x_{0}, x_{1}, \ldots\right), x_{0} \in \mathbf{R}^{M}, x_{n} \in \mathbf{C}^{M}\right\}$ with norm $\|x\|^{2}=\left\|x_{0}\right\|^{2}+\sum n^{2}\left|x_{n}\right|^{2}$. That is finding solutions of

$$
x-f(x, \nu)=x-x_{0}-K g(x, \nu)
$$

in the open bounded invariant subset $\Omega$ of $E$, defined as before, such that there are no solutions on $\partial \Omega$.

Assume that $f\left(x_{0}, \nu\right) \neq x_{0}$; i.e., there are no stationary solutions in $\Omega$. Then the $S^{1}$-degree reduces to the degree of the equivariant part.

Let $\left(e^{i \varphi} x^{0}, \nu^{0}\right)$ be an isolated orbit corresponding to $\left(y^{0}(\tau), \nu^{0}\right)$. Then

$$
\begin{aligned}
& f_{x}\left(x^{0}, \nu^{0}\right)=x_{0}+K(B(\tau) y(\tau)), \\
& f_{\nu}\left(x^{0}, \nu^{0}\right)=K g_{\nu}\left(y^{0}, \nu^{0}\right) .
\end{aligned}
$$

In what follows we shall write $f_{x}$ and $f_{\nu}$ to denote $f_{x}\left(x^{0}, \nu^{0}\right)$ and $f_{\nu}\left(x^{0}, \nu^{0}\right)$ respectively.

Now the operator $\left(\left(I-f_{x}\right) x-\mu f_{\nu},-\operatorname{Im} z_{1}\right)$ is invertible if and only if $\operatorname{dim} \operatorname{ker}(\Phi(2 \pi)-I)=1$ and $K g_{\nu} \notin \operatorname{Range}\left(I-f_{x}\right)$; that is, the equation (after applying $\left.A+P_{0}\right) \quad A x-B x=g_{\nu}$ or else $\dot{y}-B y=g_{\nu}$ has no solutions.

Let $z$ be the unique solution of the adjoint problem $\dot{z}+B^{\text {tr }} z=0$. Then, necessarily, $\int_{0}^{2 \pi} g_{\nu} \cdot z d \tau \neq 0$. If 1 is a simple eigenvalue of $\Phi(2 \pi)$, then $\dot{x}^{0}$ is not in Range $(d / d t-B)$ and $z$ can be normalized in such a way that $\int_{0}^{2 \pi} \dot{x}^{0} \cdot z d \tau=2 \pi$. Since the operator $d / d \tau-(B-\lambda)$ is invertible for $\lambda$ small and positive, the equation $\left(I-f_{x}+\lambda K\right) x=f_{\nu}$ has a unique solution $x^{\prime}$ and the index of the operator $\left(\left(I-f_{x}\right) x-\mu f_{\nu},-\operatorname{Im} z_{1}\right)$ will be the index of the operator

$$
\left(\left(I-f_{x}+\lambda K\right)\left(x-\mu x^{\prime}\right),-\operatorname{Im}\left(x-\mu x^{\prime}\right)_{1}-\mu \operatorname{Im} x_{1}^{\prime}\right) .
$$

Assuming that $\operatorname{Im} x_{1}^{\prime} \neq 0$, then $(x=0, \mu=0)$ is the only zero of the above operator. Hence, deforming $x-\mu x^{\prime}$ to $x$, and repeating the arguments given in the study of the Fuller index, we obtain the following proposition. 
Proposition 4.19. If the equation $\dot{y}-B y=0$ has the only solution $y=\dot{x}^{0}$ and $g_{\nu}$ is such that $\left(g_{\nu}, z\right) \neq 0$, where $z$ is a solution of $\dot{z}+B^{\operatorname{tr}} z=0$, then the nonstationary solution $x^{0}$ has normalized $S^{1}$-index $=-\operatorname{sign} \operatorname{Im} x_{1}^{\prime}(-1)^{\sum n_{i}} / n_{0}$, where $n_{i}$ is the multiplicity of the Floquet multiplier $\lambda_{i}>1$. If 1 is a simple Floquet multiplier, then $\operatorname{sign} \operatorname{Im} x_{1}^{\prime}=\operatorname{sign}\left(g_{\nu}, z\right)$, where $z$ is normalized by $\left(z, \dot{x}^{0}\right)=1$.

In fact, since codim $\operatorname{Range}\left(I-f_{x}\right)=1$, any two vectors $K z^{1}$ and $K z^{2}$ such that $\left(z^{1}, z\right)$ and $\left(z^{2}, z\right)$ have the same sign will give the same sign for sign $\operatorname{Im} x_{1}^{\prime}$ (replace $g_{\nu}$ by $\left.\tau z^{1}+(1-\tau) z^{2}\right)$. If 1 is simple then $\dot{x}^{0}$ gives $x^{\prime}=\lambda^{-1} A x^{0}, K A x^{0}=x^{0}-x_{0}^{0}$.

Remark 4.20. One could have defined "Floquet multipliers" in the abstract setting. In fact, suppose that $E$ is compactly contained in $E_{0}$ and that $A: E \rightarrow E_{0}$ is a Fredholm operator of index 0 , with $\operatorname{ker} A=\operatorname{coker} A=E^{S^{1}}$. Let $P_{0}$ be the projection of $E$ onto $E^{S^{1}}$, i.e., onto the constants, and let $K: E_{0} \rightarrow E$ be the map such that $\left(A+P_{0}\right) K=I_{E_{0}}$ and $K\left(A+P_{0}\right)=I_{E}$. Note that the compactness assumption implies that $\lim _{i \rightarrow+\infty} n_{i}=+\infty$. Assume that $f_{x}$ is also defined on $E_{0}$ and is continuous from $E_{0}$ into $E$ (these two last assumptions could be replaced by asking that $I-f_{x}+\lambda K$ is invertible for $\lambda$ large and positive). Finally, assume that $E_{0}$ is a Hilbert space, so that the identity $\left(e^{i \varphi} x, e^{i \varphi} x\right)=(x, x)$ implies that $(A x, x)+(x, A x)=0$; thus $(A x, x)=0$ when the operator $A$ is regarded as a real operator, if not $\operatorname{Re}(A x, x)=0$. Thus, if $T=I-f_{x}+\lambda K$ and $\widetilde{T}=\left(A+P_{0}\right) T$, then $T=K \widetilde{T}$. Since the operator $T$ is of the form $I$-compact on $E$, then it is Fredholm of index 0 for all $\lambda$. We also have that $\widetilde{T}: E \rightarrow E_{0}$ is Fredholm of index 0 and that $\tilde{T} x=A x-\left(A+P_{0}\right) f_{x} x+\lambda x$. Furthermore, $\operatorname{Re}(\tilde{T} x, x)=\lambda\|x\|^{2}-c\|x\|^{2} \geq 0$ for $\lambda \geq c$, where $c=\left\|\left(A+P_{0}\right) f_{x}\right\|$, as an operator from $E_{0}$ into itself. Thus the Fredholm operator $A-\left(A+P_{0}\right) f_{x}: E \rightarrow E_{0}$ has no eigenvalues $\lambda$, if $\lambda$ is large enough. Hence its spectrum is discrete (see $\left[\mathrm{I}_{0}, \mathrm{p}\right.$. 43]). Let $\lambda_{1}, \ldots, \lambda_{N}$ be the positive eigenvalues of $A-\left(A+P_{0}\right) f_{x}$ with algebraic multiplicity $n_{i}$ $\left(e^{2 \pi \lambda_{i}}\right.$ are the Floquet multipliers). Then, if $\left(e^{i \varphi} x^{0}, \nu^{0}\right)$ is a hyperbolic orbit, its normalized $S^{1}$-index is, as above, $-\operatorname{sign} \operatorname{Im} x_{1}^{\prime}(-1)^{\sum n_{i}}$. If 1 is a simple Floquet multiplier one obtains the same relation as before.

Remark 4.21. If there is no parameter $\nu$ and the differential equation, $y=g(y)$ has no $2 \pi$-periodic solution on $\partial \omega \subset \mathbf{R}^{M}$ then all the information is given either by $p_{*}(I-f)$ (see Theorem 4.4) or by $\operatorname{deg}(I-f, \Omega$ ) without taking into account the action (this degree is always 0 if $\Omega^{S^{1}}=\varnothing$; see Theorem 4.4(ii)).

\section{APPENDIX}

A. Definition of $\pi_{M}^{\Gamma}\left(S^{N}\right)$. Let $\Gamma$ be a compact Lie group acting linearly and isometrically on both $\mathbf{R}^{M}$ and $\mathbf{R}^{N}$. Let $\mathbf{R}^{k}=\left(\mathbf{R}^{M}\right)^{\Gamma}, \mathbf{R}^{l}=\left(\mathbf{R}^{N}\right)^{\Gamma}$ be the 
corresponding fixed-point subspaces. Thus any point $z \in \mathbf{R}^{M}$ will be written as $z=(x, y), x \in \mathbf{R}^{k}$ and $y \in \mathbf{R}^{M-k}$. We shall define the $\Gamma$-homotopy groups in a particular fashion. This is suggested to us by our construction of the $\Gamma$ degree when considering maps of the form $\left(\Phi_{0}, \Phi\right)$, where $\Phi_{0}: \mathbf{R}^{M} \rightarrow \mathbf{R}^{l}$ is $\Gamma$-invariant, $\Phi: \mathbf{R}^{M} \rightarrow \mathbf{R}^{N-l}$ is $\Gamma$-equivariant, and finally when passing to the cylinder construction by adding a real parameter. More precisely, let $B_{R}=$ $\left\{(x, y) \in \mathbf{R}^{M}:\|x\|^{2}+\|y\|^{2} \leq R^{2}\right\}$ and let $\mathscr{E}$ be the set of all $\Gamma$-equivariant maps

$$
F:[0,1] \times B_{R} \rightarrow \mathbf{R} \times \mathbf{R}^{l} \times \mathbf{R}^{N-l}, \quad F \neq 0 \text { on } \partial\left([0,1] \times B_{R}\right),
$$

of the form

$$
F(t, z)=\left(f_{0}(t, z), \Phi_{0}(t, z), \Phi(t, z)\right)
$$

where $f_{0}$ is a real-valued function, $\Phi_{0}$ is $\Gamma$-invariant, and $\Phi$ is $\Gamma$-equivariant (here $\Gamma$ acts trivially both on $[0,1]$ and on $\mathbf{R}$ ). If $k>0, l=0$, then we restrict the class $\mathscr{E}$ to maps $F$ which have $f_{0}(1,0)$ positive. If $k=0, l=0$, we take only maps $F$ which have $f_{0}(1,0)$ and $f_{0}(0,0)$ both positive. Observe that the $\Gamma$-equivariance of the map $F$ yields immediately

$$
\Phi(t, x, 0)=0 \text { for any } x \in \mathbf{R}^{k}, t \in \mathbf{R} .
$$

These mappings are divided in $\Gamma$-homotopy classes: $F \stackrel{\Gamma}{\sim} G$ if there exists a continuous $\Gamma$-homotopy

$$
H: \partial\left([0,1] \times B_{R}\right) \times[0,1] \rightarrow \mathbf{R} \times \mathbf{R}^{l} \times \mathbf{R}^{N-l} \backslash\{0\}
$$

such that

(a) $H(t, z ; 0)=F(t, z)$ and $H(t, z ; 1)=G(t, z)$ for any $(t, z) \in \partial([0,1] \times$ $\left.B_{R}\right)$;

(b) $H(\cdot ; \tau) \in \mathscr{E}$ for any $\tau \in[0,1]$.

In order to endow the set of these $\Gamma$-homotopy classes with a group structure, we shall need the following result.

Proposition A.1. For any $F \in \mathscr{E}$ there exists $G \in \mathscr{E}$ such that $F \stackrel{\Gamma}{\sim} G$ and $G(t, z)=(1,0,0)$ for all $(t, z) \in(\partial[0,1]) \times B_{R}$.

Proof. Let $A=(\partial[0,1]) \times B_{R}$. Then $A$ is a closed and $\Gamma$-invariant set. Clearly the $\Gamma$-homotopy $F(t, \tau z)$ is admissible on $A$ for any $\tau \in[0,1]$. Thus the restriction of $F$ to $A$ is $\Gamma$-homotopic to the map $H(t, z):=F(t, 0),(t, z) \in$ $A$. Due to $(*)$, the map $H$ is of the form

$$
H(t, z)=\left(f_{0}(t, 0), \Phi_{0}(t, 0), 0\right) \in \mathbf{R} \times \mathbf{R}^{l} \times \mathbf{R}^{N-l} \backslash\{0\}, \quad(t, z) \in A .
$$

Now let $G$ be the map defined by $G(t, z)=(1,0,0),(t, z) \in A$. We will show that, under the above assumptions, the maps $H$ and $G$ are $\Gamma$-homotopic. Indeed,

(i) If $l>0$, then $H(t, z) \in\left(\mathbf{R} \times \mathbf{R}^{l} \backslash\{0\}\right) \times\{0\}$, for any $(t, z) \in A$. Thus, taking a path in $\mathbf{R}^{l+1} \backslash\{0\}$, we have that $H \stackrel{\Gamma}{\sim} G$ on $A$. 
(ii) If $l=0$ and $k>0$, then $H(t, z)=\left(f_{0}(t, 0), 0\right) \neq 0$, for any $(t, z) \in$ $A$. Since $F \in \mathscr{E}$, it turns out that its restriction to the set

$$
\partial\left\{(t, z) \in[0,1] \times B_{R}: z=x\right\}
$$

is a continuous map from $S^{k}$ into $\mathbf{R} \backslash\{0\}$. Thus, in particular, the map $f_{0}(t, 0)$ is positive. Hence $H \stackrel{\Gamma}{\sim} G$ on $A$.

(iii) If $l=k=0$, then $H$ is $\Gamma$-homotopic to $G$ since $f_{0}(0,0)$ and $f_{0}(1,0)$ are both positive, by assumption.

Now, using the $\Gamma$-equivariant Borsuk homotopy extension theorem, the map $F$ will be $\Gamma$-homotopic to a map in $\mathscr{E}$ which extends the map $G$ to all of $\partial\left([0,1] \times B_{R}\right)$. Q.E.D.

We shall denote by $\pi_{M}^{\Gamma}\left(S^{N}\right)$ the totality of $\Gamma$-homotopy classes of maps in $\mathscr{E}$. We shall also denote by $[F]_{\Gamma}$ the class which contains the map $F$ and by $0_{\Gamma}$ the class containing the constant map $c$ with value $(1,0,0)$ on $[0,1] \times B_{R}$.

Remark A.2. The class $0_{\Gamma}$ is also the class of all maps $F$ which have a nonvanishing $\Gamma$-equivariant extension to the cylinder $[0,1] \times B_{R}$. Indeed, if one has a nonzero $\Gamma$-equivariant extension $\bar{F}$ of $F$ to the above cylinder, then using the deformation $(\tau t, \tau z)$ the map $F$ is $\Gamma$-homotopic to the constant map $\left(f_{0}(0,0), 0,0\right)$, which is clearly homotopic to $c$. The converse is just a direct consequence of the Borsuk homotopy extension theorem for $\Gamma$-equivariant maps.

To proceed further we need a concept of addition on $\pi_{M}^{\Gamma}\left(S^{N}\right)$. To this end let $F$ and $G$ be any two maps belonging to $\mathscr{E}$. By virtue of Proposition A.1 we may assume that $F_{\mid A}=G_{\mid A}=(1,0,0)$. Define their sum $F \oplus G$ as the map

$$
F \oplus G(t, z)= \begin{cases}F(2 t, z) & \text { if } 0 \leq t \leq \frac{1}{2} \text { and } z \in B_{R}, \\ G(2 t-1, z) & \text { if } \frac{1}{2} \leq t \leq 1 \text { and } z \in B_{R} .\end{cases}
$$

Clearly the $\Gamma$-homotopy class $[F \oplus G]_{\Gamma}$ depends only on the two classes $[F]_{\Gamma}$ and $[G]_{\Gamma}$. Hence we have the following.

Definition A.3. The addition in $\pi_{M}^{\Gamma}\left(S^{N}\right)$ is given by

$$
[F]_{\Gamma}+[G]_{\Gamma}=[F \oplus G]_{\Gamma}
$$

This addition turns out to be associative (see $[G, p .7]$ ), the class $0_{\Gamma}$ is the neutral element of the group, and the inverse element of $[F]_{\Gamma}$ is the class $[F \circ \theta]_{\Gamma}$, where $\theta(t, z)=(1-t, z)$ for any $(t, z) \in[0,1] \times B_{R}$. Therefore, $\pi_{M}^{\Gamma}\left(S^{N}\right)$ is a group under the addition defined above.

Moreover, the following result holds.

Proposition A.4. The group $\pi_{M}^{\Gamma}\left(S^{N}\right)$ is abelian, provided $k \geq 1$.

Proof. First, let us observe that if we write $z \in B_{R}$ as $z=(x, y)$ with $x=$ $\left(x_{0}, \tilde{x}\right), x_{0} \in \mathbf{R}$, then in the $\Gamma$-homotopy class $[F]_{\Gamma}$ we can always choose a 
map with value $(1,0,0)$ on the set $A^{+}=\left\{(t, z) \in[0,1] \times \partial B_{R}: x_{0} \geq 0\right\}$ (we can choose as well a map with value $(1,0,0)$ on the set $A^{-}=\{(t, z) \in[0,1] \times$ $\left.\left.\partial B_{R}: x_{0} \leq 0\right\}\right)$. Indeed, let $A$ be the set defined by $A=A^{+} \cup(\partial[0,1]) \times B_{R}$. Then $A$ is closed and $\Gamma$-invariant. Let $F \in \mathscr{E}$ and let $G(t, z)=(1,0,0)$ for $(t, z) \in A$. Then the restriction $F_{\mid A}$ of $F$ to $A$ and the map $G$ are $\Gamma$-homotopic, via the homotopy defined as follows:

$$
H(t, z ; \tau)= \begin{cases}F\left(t, \frac{x_{0} \cos \tau \pi / 2+R \sin \tau \pi / 2}{\alpha(\tau)}, \frac{\tilde{x} \cos \tau \pi / 2}{\alpha(\tau)}, \frac{y \cos \tau \pi / 2}{\alpha(\tau)}\right) \\ \text { if }(t, z) \in A^{+} \text {and } \tau \in[0,1],\end{cases}
$$

where $\alpha(\tau)=\left(1+R^{-1} x_{0} \sin \pi \tau\right)^{1 / 2}$.

Thus, using the $\Gamma$-equivariant Borsuk homotopy extension theorem, the map $F$ is $\Gamma$-homotopic to a map having value $(1,0,0)$ on $A^{+}$.

Now we are in a position of proving that $\pi_{M}^{\Gamma}\left(S^{N}\right)$ is abelian. Indeed, consider two maps $F_{1}$ and $F_{2}$ such that

$$
F_{1}(t, z)=(1,0,0) \text { for any }(t, z) \in A^{+}
$$

and

$$
F_{2}(t, z)=(1,0,0) \text { for any }(t, z) \in A^{-} .
$$

Define the $\Gamma$-equivariant homotopy

$$
H(t, z ; \tau)= \begin{cases}F_{1}(2 t-\tau, z) & \text { for }(t, z) \in A^{-}, 0 \leq 2 t-\tau \leq 1, \\ F_{2}(2 t-(1-\tau), z) & \text { for }(t, z) \in A^{+}, 0 \leq 2 t-(1-\tau) \leq 1, \\ (1,0,0) & \text { otherwise. }\end{cases}
$$

Simple computations give that $H$ is admissible. Moreover,

$$
H_{0} \in\left[F_{1}\right]_{\Gamma}+\left[F_{2}\right]_{\Gamma} \text { and } H_{1} \in\left[F_{2}\right]_{\Gamma}+\left[F_{1}\right]_{\Gamma} .
$$

Thus $\pi_{M}^{\Gamma}\left(S^{N}\right)$ is an abelian group. Q.E.D.

The following remarks are in order.

Remark A.5. The addition used in the additivity property of the $\Gamma$-degree (see Property (e) in $\S 2$ ) coincides with that of Definition A.3.

To see this, let $F$ and $F_{1}$ be as in Property (e); that is, $F, F_{1}:[0,1] \times B_{R} \rightarrow$ $\mathbf{R}^{N+1}$ are $\Gamma$-equivariant, nonvanishing on $\partial\left([0,1] \times B_{R}\right)$, and $F(t, z)=F_{1}(t, z)$ for $(t, z) \in\left[\frac{1}{2}, 1\right] \times B_{R}$. By the definition of the sum in $\pi_{M}^{\Gamma}\left(S^{N}\right)$, the $\Gamma$ homotopy class $[F]_{\Gamma}-\left[F_{1}\right]_{\Gamma}$ is induced by the map

$$
h(t, z)= \begin{cases}F(2 t, z), & 0 \leq t \leq \frac{1}{2}, z \in B_{R}, \\ F_{1}(2-2 t, z), & \frac{1}{2} \leq t \leq 1, z \in B_{R} .\end{cases}
$$

Now consider the $\Gamma$-homotopy

$$
H_{\tau}(t, z)= \begin{cases}F((2-\tau) t, z), & 0 \leq t \leq \frac{1}{2}, z \in B_{R} \\ F_{1}((2-\tau)(1-t), z), & \frac{1}{2} \leq t \leq 1, z \in B_{R}\end{cases}
$$


Clearly, $H_{\tau}$ is admissible. Hence $[h]_{\Gamma}=\left[H_{0}\right]_{\Gamma}=\left[H_{1}\right]_{\Gamma}$. But $H_{1}$ is nothing else than the map (2.3) introduced in Property (e).

Remark A.6. Let $\Omega$ be a bounded open $\Gamma$-invariant subset of $\mathbf{R}^{M}$ and let $f: \bar{\Omega} \rightarrow \mathbf{R}^{N}$ be a $\Gamma$-equivariant map such that $\operatorname{deg}_{\Gamma}(f, \Omega)$ is defined. Assume that $\Omega^{\Gamma} \neq \varnothing$. Then the map $f$ can be decomposed as $f=\left(\Phi_{0}, \Phi\right)$ with $\Phi_{0}(z) \in \mathbf{R}^{l}=\left(\mathbf{R}^{N}\right)^{\Gamma}$ and $\Phi(z) \in \mathbf{R}^{N-l}$ for any $z \in \bar{\Omega}$. Decompose $z \in \mathbf{R}^{M}$ as $z=(x, y)$ with $x \in\left(\mathbf{R}^{M}\right)^{\Gamma}=\mathbf{R}^{k}$ and $y \in \mathbf{R}^{M-k}$.

Since $f \neq 0$ on $\partial \Omega$ and $\Phi(x, 0)=0$ for any $(x, 0) \in \bar{\Omega}$, we have that $\Phi_{0}(x, 0) \neq 0$ for any $(x, 0) \in \partial \Omega^{\Gamma}$. It follows that $\operatorname{deg}_{\{e\}}\left(\Phi_{0 \mid} \bar{\Omega}^{\Gamma}, \Omega^{\Gamma}\right)$ is also defined and is an element of $\pi_{k}\left(S^{l}\right)$. Therefore, $\operatorname{deg}_{\{e\}}\left(\Phi_{0 \mid} \overline{\boldsymbol{\Omega}}^{\Gamma}, \Omega^{\Gamma}\right) \neq 0_{\{e\}}$ implies $\operatorname{deg}_{\Gamma}(f, \Omega) \neq 0_{\Gamma}$.

Remark A.7. Let $\Omega$ and $f$ be as above. Moreover assume that $k=l=0$. By Proposition A.1 it follows that in order to be able to use the group structure of $\pi_{M}^{\Gamma}\left(S^{N}\right)$ one has to impose the extra assumption $0 \notin \bar{\Omega}$. As already observed in $\S 4$, this is one of the basic assumptions when defining both Fuller's and Dancer's degrees.

B. The $\Gamma$-equivariant Freudenthal suspension theorem. In this part of the Appendix we first state an equivariant Freudenthal suspension theorem in a form which is suitable for our purposes and then elucidate its meaning for specific examples.

We begin with some definitions. Let $\Gamma$ be a compact Lie group acting linearly and isometrically on $\mathbf{R}^{M}, \mathbf{R}^{N}, \mathbf{R}^{P}$, and $\mathbf{R}^{v}=V$, respectively. Let Iso $\left(S^{M+P}\right)$ denote the set of all isotropy subgroups of points in $S^{M+P}$-the boundary of the unit ball in $\mathbf{R} \times \mathbf{R}^{M} \times \mathbf{R}^{P}$ (we assume tacitly that the action of $\Gamma$ on the first factor $\mathbf{R}$ is trivial). If $(t, x, y)$ is any point in $\mathbf{R} \times \mathbf{R}^{M} \times \mathbf{R}^{P}$, the fact that the action is linear implies that $\Gamma_{(t, x, y)}=\Gamma_{(t, x, 0)} \cap \Gamma_{(t, 0, y)}$ and that $\operatorname{Iso}\left(S^{M}\right) \subset \operatorname{Iso}\left(S^{M+P}\right)$. If $H \in \operatorname{Iso}\left(S^{M+P}\right)$, then we set

$$
\begin{array}{ll}
k=\operatorname{dim}\left(\mathbf{R}^{M}\right)^{\Gamma}, & m^{H}=\operatorname{dim}\left(\mathbf{R}^{M}\right)^{H}=k+z^{H}, \quad p^{H}=\operatorname{dim}\left(\mathbf{R}^{P}\right)^{H}, \\
l=\operatorname{dim}\left(\mathbf{R}^{N}\right)^{\Gamma}, & n^{H}=\operatorname{dim}\left(\mathbf{R}^{N}\right)^{H}=l+y^{H}, \quad \text { and } \quad v^{H}=\operatorname{dim}\left(\mathbf{R}^{v}\right)^{H} .
\end{array}
$$

If $H=\{e\}$, then of course $m^{e}=M, p^{e}=P, n^{e}=N$, and $v^{H}=v$.

Finally, let $F$ be an element of $\mathscr{E}$, that is, in the class of all $\Gamma$-equivariant maps defined on $[0,1] \times B^{M+P}$ with values on $\mathbf{R} \times \mathbf{R}^{N} \times \mathbf{R}^{P}$ and nonzero on $S^{M+P}=\partial\left([0,1] \times B^{M+P}\right)$ (with the restriction on the signs of $f_{0}(0,0)$ and $f_{0}(1,0)$ when $\left.l=0\right)$. Thus, according to $\S \mathrm{A}$ of this Appendix, we may consider the $\Gamma$-homotopy class $[F]_{\Gamma}$ of the map $F$ as an element of the group $\pi_{M+P}^{\Gamma}\left(S^{M+P}\right)$. Moreover, for $V=\mathbf{R}^{v}$ we define the $V$ - $\Gamma$-equivariant suspension $\Sigma^{V}(F)$ of the map $F$ as

$$
\Sigma^{V}(F)(t, z, v)=(F(t, z), v), \quad \text { if }(t, z, v) \in[0,1] \times B^{M+P} \times V .
$$


Clearly, $\Sigma^{V}$ defines a group homomorphism

$$
\begin{gathered}
\Sigma^{V}: \pi_{M+P}^{\Gamma}\left(S^{N+P}\right) \rightarrow \pi_{M+P+v}^{\Gamma}\left(S^{M+P+v}\right), \\
{[F]_{\Gamma} \mapsto\left[\Sigma^{V}(F)\right]_{\Gamma} .}
\end{gathered}
$$

We are now in a position to state the following version of the $\Gamma$-equivariant Freudenthal suspension theorem (see [H, Theorem 2.4] and [Na, Theorem 2.3]).

Theorem B. The equivariant suspension homomorphism $\Sigma^{V}$ is an isomorphism if the following conditions hold:

(i) if $H \in \operatorname{Iso}\left(S^{M+P}\right)$ with $v^{H}>0$, then $p^{H} \geq k-2 l+z^{H}-2 y^{H}+2$;

(ii) if $K, H \in \operatorname{Iso}\left(S^{M+P}\right)$ with $K<H$ and $v^{K}>v^{H}$, then $p^{K}-p^{H} \geq$ $k-l+z^{H}-y^{K}+2$.

We shall now show the use of this suspension theorem when some particular representation of $\Gamma$ is chosen.

Example B.1. Assume that $\Gamma$ acts trivially on $V\left(=\mathbf{R}^{v}\right)$. Thus $\Sigma^{V}$ is the usual suspension homomorphism when no action is present. In this case for any subgroup $H$ of $\Gamma$ we have that $v^{H}=v$. Hence condition (ii) of Theorem B is void. Assume moreover that $\Gamma$ also acts trivially on $\mathbf{R}^{P}$ and that the following dimensional inequality holds:

$$
P \geq M-2 l+2
$$

(or $P \geq k-2 l+2$ if $z^{H}=y^{H}$ for any $H \in \operatorname{Iso}\left(S^{M}\right)$ ).

By Theorem B, we have that $\Sigma^{V}$ is an isomorphism. Thus we may always stabilize the $\Gamma$-homotopy group by taking trivial actions.

In particular, under the above assumptions, the $\Gamma$-degree tas the complete addivity property; i.e., no suspension of maps is needed (see $\S 2$ ).

Furthermore, for $\Gamma$-equivariant maps $f: S^{M-1} \rightarrow \mathbf{R}^{N} \backslash\{0\}$ (as in the suspension property in $\S 2$ ) the one-dimensional forgetful suspension $\Sigma$ (forgetting equivariance)

$$
\Sigma(f)\left(x_{1}, \ldots, x_{M}, x_{M+1}\right)=\left(f\left(x_{1}, \ldots, x_{M}\right), x_{M+1}\right)
$$

becomes an isomorphism provided that

(1) $k-2 l+3+z^{H}-2 y^{H} \leq 0$ for any $H \in \operatorname{Iso}\left(S^{M-1}\right)$.

Clearly (1) implies condition (i) of Theorem B. Note that (1) holds if either $M-2 l+3 \leq 0$ or else $z^{H}=y^{H}$ for any $H \in \operatorname{Iso}\left(S^{M-1}\right)$ and $k-2 l+3 \leq 0$.

Example B.2. Now consider the case $\mathbf{R}^{P}=r V \quad(r$-copies of $V)$ with

(2) $r \geq M-l+2$ (or $r \geq k-l+2$, if $z^{H}=y^{H}$ for all $H \in \operatorname{Iso}\left(S^{M+P}\right)$ ).

Then, according to Theorem $\mathrm{B}, \Sigma^{V}$ is an isomorphism. Indeed, the dimensional assumption (2) implies (i) since for any $H \in \operatorname{Iso}\left(S^{M+P}\right)$ we have $M \geq k+z^{H}$ and $p^{H}=r v^{H} \geq r$ if $v^{H}>0$. Moreover, given $K, H \in \operatorname{Iso}\left(S^{M+P}\right)$ 
such that $K<H$ and $v^{K}-v^{H}>0$ then $p^{K}-p^{H}=r\left(v^{K}-v^{H}\right) \geq r$. Hence condition (ii) of Theorem B is also satisfied (note that $y^{H} \leq y^{K}$ if $K<H$ ).

It is clear then that a way to stabilize the $\Gamma$-degree, in the infinite-dimensional case, is to insert sufficiently many copies of $V(M-l+2$ additional copies $)$ into $\mathbf{R}^{P}$.

Example B.3. We now consider the case of an $S^{1}$-almost semifree action. Let $\left(\mathbf{R}^{M}\right)^{S^{1}}=\mathbf{R}^{k},\left(\mathbf{R}^{N}\right)^{S^{1}}=\mathbf{R}^{l}$, and $\mathbf{R}^{M}=\mathbf{R}^{k} \times \mathbf{C}^{m}, \mathbf{R}^{N}=\mathbf{R}^{l} \times \mathbf{C}^{n}$ being the action on $\mathbf{C}^{m}$ :

$$
\mathbf{e}^{i m_{0} \varphi}:=\left(e^{i m_{0} \varphi} z_{1}, \ldots, e^{i m_{0} \varphi} z_{m}\right)
$$

and on $\mathbf{C}^{n}$

$$
\mathbf{e}^{i n \varphi} \xi=\left(e^{i n_{1} \varphi} \xi_{1}, \ldots, e^{i n_{n} \varphi} \xi_{n}\right)
$$

where $n_{1}, \ldots, n_{n}$ are multiples of $m_{0}$. Thus the only isotropy subgroups are $S^{1}$ or $\mathbf{Z}_{m_{0}}\left(\{e\}\right.$ if $\left.m_{0}=1\right)$. We make the additional assumption that either $m=1$ or $m \leq n+1-(k-l) / 2$.

Let $\mathbf{R}^{P}=\{0\}$ and $V=\mathbf{R}$. Then the second condition in the theorem is void and the first reads as:

$$
k \leq 2 l-2 \text { for } H=S^{1} \text { and } k \leq 2 l-2+4 n-2 m \text { if } H=\mathbf{Z}_{m_{0}} .
$$

It is clear that both conditions can be met by adding enough copies of $\mathbf{R}$. Now for $m=1$ one is reduced to $k \leq 2 l-2$ if $n \geq 1, k \leq 2 l-4$ if $n=0$. If $1<m \leq n+1-(k-l) / 2$, the second condition is always satisfied provided that $k \leq 2 l-2$ and $2 n+l \geq 3$ if $|k-l|$ is odd and $2 n+l \geq 4$ if $|k-l|$ is even. But if $|k-l|$ is odd and $m \geq 1$ then $k-l \leq 2 n-3$, while if $|k-l|$ is even and $m>1$ then $k-l \leq 2 n-2$. Thus $\Sigma$ is always an isomorphism provided that $k \leq 2 l-2$ except for $m=1, n=0$; then one needs $k \leq 2 l-4$ and $n=0, m=2, l=k+2=2$ or 3 . By adding $\mathbf{R}^{r}$ one may achieve this isomorphism if $r \geq k-2 l+2(r \geq k-2 l+4$ if $n=0, m=1 ; r=4-l$ if $n=0, m=2, l=k+2=2$ or 3 ). If $k=l+1, m=n$ then one is in the stable range if $l \geq 3$.

If $\mathbf{R}^{P}=\{0\}$ and $V=\mathbf{C}$ with action $\mathbf{e}^{i m_{0} \varphi}$, then the first condition reads as $k \leq 2 l-2+4 n-2 m$ and the second one as $k \leq l+2 n-2$. If $m=1$ one needs only $k \leq l+2 n-2$. If $m>1$ and $|k-l|$ is odd then $k \leq l+2 n-2$ is the only condition. If $m>1$ and $|k-l|$ is even then $k \leq l+2 n-2$ and $k \geq 2$ (this excludes the cases $k=0, l=0, n=1, m=2 ; k=0, n=0, l=2, m=2$; $k=1, l=1, n=1, m=2 ; k=1, l=3, n=0, m=2)$. By adding $\mathbf{C}^{s}$ with action $\mathbf{e}^{i m_{0} \varphi}$, one will get an isomorphism for $2 s \geq k-l-2 n+2 \quad(s=2$ for the cases $n=0, m=1, k=l=0$ or 1$)$. If $k=l+1, m=n$, one is in the stable range as soon as $n>1$, as noticed in [I.M.V] for the case of the degrees defined by Fuller and Dancer.

Note that our results in $\S 4$, by looking directly at the groups, are better. 
Example B.4. Let $\Gamma=S^{1}$ be the group of complex numbers of norm 1 and let the actions be described as follows:

$$
\begin{aligned}
\mathbf{e}^{i m \varphi}\left(x, z_{1}, \ldots, z_{m}\right) & =\left(x, e^{i m_{1} \varphi} z_{1}, e^{i m_{2} \varphi} z_{2}, \ldots, e^{i m_{m} \varphi} z_{m}\right), \\
\mathbf{e}^{i n \varphi}\left(x, z_{1}, z_{2}, \ldots, z_{n}\right) & =\left(x, e^{i n_{1} \varphi} z_{1}, e^{i n_{2} \varphi} z_{2}, \ldots, e^{i n_{n} \varphi} z_{n}\right),
\end{aligned}
$$

both on $\mathbf{R}^{M}=\mathbf{R}^{k} \times \mathbf{C}^{m}$ for $x \in \mathbf{R}^{k}$ and $z_{i} \in \mathbf{C}, i=1, \ldots, m$, and on $\mathbf{R}^{N}=\mathbf{R}^{l} \times \mathbf{C}^{n}$ for $x \in \mathbf{R}^{l}$ and $z_{i} \in \mathbf{C}, i=1, \ldots, n$.

On $\mathbf{R}^{P}=\mathbf{R}^{r} \times \mathbf{C}^{s}$, for $\left(z_{1}, \ldots, z_{s}\right) \in \mathbf{C}^{s}$, let the action be given by

$$
\mathbf{e}^{i s \varphi} z=\left(e^{i S_{1} \varphi} z_{1}, \ldots, e^{i S_{s} \varphi} z_{s}\right)
$$

Here $\mathbf{R}^{P}$ is a finite-dimensional subspace of an infinite-dimensional $S^{1}$-Banach space $E$.

First, note that if $H \in \operatorname{Iso}\left(S^{M+P}\right)$ then $H$ is either $\{e\}$ or a finite cyclic group or the whole group $S^{1}$. Let $\mathscr{H}$ be the subset of the subgroups of $S^{1}$ consisting of $S^{1},\{e\}, \mathbf{Z}_{q}$ where $q$ divides one of the $m_{1}, \ldots, m_{m}$. Thus $\mathscr{H}$ contains $\operatorname{Iso}\left(S^{M}\right)$ and all subgroups of the finite groups in $\operatorname{Iso}\left(S^{M}\right)$. For $H$ in $\mathscr{H}$ one may assume that whenever $v^{H}>0$ or $v^{K}>v^{H}$, if $K<H$, for some finite-dimensional subspace $V$ of $E$ the conditions (i) and (ii) are satisfied: in fact, if $p^{H}<k-2 l+z^{H}-2 y^{H}+2$ then add to $\mathbf{R}^{P}$ the subspace $V$; this will increase $p^{H}$ by $\operatorname{dim}_{\mathbf{R}} V$. Thus one will get to the point where either $p^{H} \geq k-2 l+z^{H}-2 y^{H}+2$ or there is no subspace of $E$ such that $v^{H}>0$. Similarly, if $p^{K}-p^{H}<k-l+z^{H}-y^{K}+2$, adding $V$ will increase $p^{K}-p^{H}$ by $v^{K}-v^{H}$ and the same phenomenon will happen.

Let $H$ be in Iso $\left(S^{M+P}\right)$; then one has to check the inequalities only if $H$ does not belong to $\mathscr{H}$. Then $z^{H}=0, H$ is not $S^{1}$, and $H \in \operatorname{Iso}\left(S^{P}\right)$, $p^{H} \geq 2+p^{S^{1}}, p^{H} \geq k-2 l+2$ if $v^{H}>0$, and $p^{K}-p^{H} \geq k-l+2$ if $v^{K}>v^{H}$ but then $p^{K}-p^{H} \geq 2$. By adding $[(k-l) / 2]$ (that is, $(k-l) / 2$ if $|k-l|$ is even or $(k-l+1) / 2$ if $|k-l|$ is odd) copies of $E$, one will get $\tilde{p}^{K}-\tilde{p}^{H} \geq k-l+2$ and $\tilde{p}^{H} \geq(k-l+2) p^{S^{1}} / 2+k-l+2 \geq k-2 l+2$.

Note that if one seeks nonconstant periodic solutions of autonomous differential equations, one has to consider at least two-dimensional systems: $p^{K}-p^{H} \geq$ $4, p^{H} \geq 2, k=l+1, l \geq 2$; one is directly in the stable range. Note also that for the additivity property of the degree, one needs $\operatorname{dim} E^{S^{1}} \geq k-2 l+2$. If this is not the case, one may add $\mathbf{R}^{P}$ on both sides with $p=k-2 l+2-\operatorname{dim} E^{S^{1}}$. C. Results on $\pi_{M}^{\Gamma}\left(S^{N}\right)$. In this section we gather some facts on the structure of $\pi_{M}^{\Gamma}\left(S^{N}\right)$. We shall restrict our attention to results which are of interest mainly for the generalized $\Gamma$-degree introduced in this paper.

The first theorem is an analog of the well-known classical results in ordinary homotopy theory (when no group action is present). Namely, $\pi_{M}\left(S^{N}\right)=0$ for $M<N$. 
Theorem C.1. Assume that $k<$ l. If $z^{H}<y^{H}$ for any $H \in \operatorname{Iso}\left(S^{M}\right)$, then $\pi_{M}^{\Gamma}\left(S^{N}\right)=0_{\Gamma}$.

A proof of Theorem C.1, when $\Gamma$ is a finite group, can be found in [A, Proposition 2.4]. To prove Theorem C.1 for the case when $\Gamma$ is a general compact Lie group one has to modify the proof given in [K, Proposition 2.12] and use the obstruction theory developed in [t.D, §8.3].

The next result is a version of the Hopf classification theorem for $\Gamma$-equivariant homotopy groups. We first need some preliminary notation. Let $H$ be a subgroup of $\Gamma$. Then $|W H|$ will denote the order of the Weyl group $W H=$ $N(H) / H$, where $N(H)$ is the normalizer of $H$ in $\Gamma$. By $\operatorname{deg}_{B} f^{H}$ we simply mean the Brouwer degree of the restriction $f^{H}:\left(S^{M}\right)^{H} \rightarrow\left(S^{M}\right)^{H}$ of a $\Gamma$ equivariant map $f: S^{M} \rightarrow S^{M}$.

Theorem C.2 [t.D, Theorem 8.4.1]. Assume that $k=l \geq 1$ and

(i) $z^{H}=y^{H}$ for any $H \in \operatorname{Iso}\left(S^{M}\right)$;

(ii) $z^{H}+2 \leq z^{K}$ for any $K, H \in \operatorname{Iso}\left(S^{M}\right)$ with $K<H$.

Then $\pi_{M}^{\Gamma}\left(S^{M}\right) \neq 0_{\Gamma}$, and $\pi_{M}^{\Gamma}\left(S^{M}\right)$ is characterized by

$$
\left\{\operatorname{deg}_{B} f^{H}: H \in \operatorname{Iso}\left(S^{M}\right) \text { with }|W H|<+\infty\right\} \text {. }
$$

Furthermore, if $K, H \in \operatorname{Iso}\left(S^{M}\right)$ with $K<H$ then $\operatorname{deg}_{B} f^{K}$ is determined by $\operatorname{deg}_{B} f^{H}$ modulo $|W H|$, in the sense that fixing these $\operatorname{deg}_{B} f^{H}$ all possible $\operatorname{deg}_{B} f^{K}$ for $K<H$ fill the whole residue class modulo $|W H|$.

We now wish to discuss Theorem C.2 in the special case when $\Gamma=S^{1}$ acts linearly on $\mathbf{R}^{M}$. The commutativity of $S^{1}$ yields that $N(H)=S^{1}$ for any isotropy subgroup $H$ of $S^{1}$. Hence the only $H$ for which the order of $W H$ is finite is $S^{1}$ itself.

Assume that $k=l \geq 1$. The arguments used in Examples B.3 and B.4 give that assumption (i) of Theorem C.2 is satisfied and that $z^{H}+2 \leq z^{K}$ for any $K, H \in \operatorname{Iso}\left(S^{M}\right), K<H$. Hence (ii) of Theorem C. 2 is also satisfied. Let $f(x, z)=\left(f_{0}(x, z), f_{\infty}(x, z)\right)$ be an $S^{1}$-equivariant map where $f_{0}$ is the $S^{1}$ invariant part of $f$ on $\left(S^{M}\right)^{S^{1}}$ and $x \in\left(S^{M}\right)^{S^{1}}$. Then $\operatorname{deg}_{S^{1}}(f)$ is uniquely determined by the Brouwer degree of the map $f_{0}$. Thus the map $f$ is $\Gamma$ homotopic to the map $\left(f_{0}(x, 0), z\right)$ since both maps have the same $S^{1}$-degree. In particular, the Brouwer degree of the map $f$ (forgetting the equivariance) can be computed (see [I.M.V, Proposition 2.1]) and on the basis of the above arguments it turns out to be the Brouwer degree of the map $f_{0}$.

If, in Theorem C.2, the only assumptions are $k=l$ and (i), then a similar complete characterization of $\pi_{M}^{\Gamma}\left(S^{M}\right)$ can be found in [H.1, Theorem 4.5] in terms of the degrees of mappings associated to the isotropy subgroups $H$ which have their Weyl group of finite order. On this subject we also refer to [Da.1], where a correct version of a result of Rubinstein is given. 
We close this section by making two final remarks.

If $\Gamma$ is a finite group, then one can stabilize the group $\pi_{*}^{\Gamma}\left(S^{*}\right)$ using the category of all "allowable" representations of $\Gamma$ (this can be done as in Example B.2), and by [A, Theorem 4.3], the stable group is a finitely generated abelian group. Moreover, if one forgets the group action on $S^{M}$ (not on the suspension) then $\pi_{*}^{\Gamma}\left(S^{N}\right) \simeq \pi_{*}\left(S^{N} / \Gamma\right)$. This is [A, Theorem 5.4]. However, it is not clear to us if this sort of results are of any interest for our problems.

Finally, in the case of a general compact Lie group $\Gamma$ there is a theoretical way to compute the stable $\Gamma$-homotopy groups (see [t.D, §8.3]). However, this involves the total space of the universal principal bundle associated to $W H$, which is not easy to handle.

D. Proofs of $\S 4$. Since the proof of Theorem 4.6 is rather long, we shall split it in a few steps. We start with the following.

Lemma D.1. Let $\widetilde{F}_{1}, \widetilde{F}_{2}$ be two extensions of $F$ such that $\widetilde{F}_{1} \sim \widetilde{F}_{2}$ on $C=$ $I \times B_{0} \times\{Z=0\}$ relative to $\partial C$. Then

$$
\widetilde{F}_{1} \stackrel{S^{1}}{\sim} \widetilde{F}_{2} \text { on }\left\{\begin{array}{r}
A_{1}=I \times B_{0} \times\left(B \cap\left\{Z_{h_{c}}=\cdots=Z_{m}=0\right\}\right) \\
\text { relative to } \partial A_{1}, \text { if }|k-l| \text { odd }, \\
A_{2}=I \times B_{0} \times\left(B \cap\left\{Z_{h_{c}-1}=\cdots=Z_{m}=0\right\}\right) \\
\text { relative to } \partial A_{2}, \text { if }|k-l| \text { even } .
\end{array}\right.
$$

Proof. Let $h(x, t)$ denote the homotopy between $\widetilde{F}_{1}$ and $\widetilde{F}_{2}$. For $i=1,2$, set $X_{i}=A_{i} \times\{0\} \cup C \times[0,1] \cup A_{i} \times\{1\}$ and let $H_{i}: X_{i} \rightarrow \mathbf{R} \times \mathbf{R}^{l} \times \mathbf{C}^{n} \backslash\{0\}$ be defined by

$$
H_{i}(x, \tau)= \begin{cases}\widetilde{F}_{1}(x) & \text { if } x \in A_{i}, \tau=0, \\ h(x, \tau) & \text { if }(x, \tau) \in C \times[0,1] \\ \widetilde{F}_{2}(x) & \text { if } x \in A_{i}, \tau=1 .\end{cases}
$$

The problem of extending the homotopy $H_{i}$ is similar to the one studied at the beginning of $\S 4$ with $k$ now replaced by $k+1$. Thus, if $k+2 h<l+2 n$ the map $H_{i}$ can be extended to $A_{i} \times[0,1]$. Hence, the assertion holds for any $h \leq h_{c}-1$ in the case that $|k-l|$ is odd and for any $h \leq h_{c}-2$ if $|k-l|$ is even. Q.E.D.

Remark D.2. At this point, if $|k-l|$ is odd or $k-l \geq 2 n-1$, then $\operatorname{deg}_{K}(F)$ could depend at most only on the first extension $\left(\tilde{f}_{0}, \widetilde{\Phi}_{0}\right)$ of the invariant part of $F$. This has been used in [I.M.V].

For the moment, we shall denote by $\operatorname{deg}_{K}(F, \widetilde{F})$ the degree of the map $F$ obtained from the extension $\widetilde{F}=\widetilde{F}_{h}$ (i.e., the homotopy class of $F_{h}$ ).

Lemma D.3. (a) If $F$ and $G$ are representatives of the same class $[F]_{S^{1}}$ in $\pi_{k+2 m, \cdot}^{S^{1}}\left(S^{l+2 n}\right)$, then the sets

$$
\left\{\operatorname{deg}_{K}(F, \widetilde{F})\right\} \quad \text { and } \quad\left\{\operatorname{deg}_{K}(G, \widetilde{G})\right\}
$$

are equal. Hence we will denote them by $\left\{\operatorname{deg}_{K}([F],[\widetilde{F}])\right\}$. 
(b) In computing $\left\{\operatorname{deg}_{K}([F],[\widetilde{F}])\right\}$ one may assume that the restriction of $F$ to $\partial\left(I \times B_{0} \times\left(B \cap\left\{Z_{h_{c}}=\cdots=Z_{m}=0\right\}\right)\right.$ is of the form $(1,0,0)$. All the different degrees are classified by $\chi_{*}([\widetilde{F}])$, with $[\widetilde{F}] \in \pi_{k+1+2\left(h_{c}-1\right),}^{S^{1}}\left(S^{l+2 n}\right)$.

(c) $\left\{\operatorname{deg}_{K}([F]+[G],[\widetilde{F}]+[\widetilde{G}])\right\}=\left\{\operatorname{deg}_{K}([F],[\widetilde{F}])\right\}+\left\{\operatorname{deg}_{K}([G],[\widetilde{G}])\right\}$.

(d) $\left\{\operatorname{deg}_{K}([F],[\widetilde{F}])\right\}$ reduces to a single element; that is, $\left\{\operatorname{deg}_{K}([F],[\widetilde{F}])\right\}=$ $\operatorname{deg}_{K}([F])$ is independent of $\widetilde{F}$, if and only if $\left\{\operatorname{deg}_{K}([0],[\widetilde{0}])\right\}=0$.

Proof. (a) Let $F \stackrel{S^{1}}{\sim} G$ on $\partial\left(I \times B_{0} \times B\right)$, let $h_{\tau}$ denote the $S^{1}$-homotopy joining $F$ and $G$, and let $\widetilde{F}$ be an extension of $F$ to $I \times B_{0} \times\left(B \times\left\{Z_{h_{c}}=\right.\right.$ $\left.\cdots=Z_{m}=0\right\}$ ). By the equivariant Borsuk extension theorem, the map $G$ will also extend to $\widetilde{G}$ on the same set and $\widetilde{F} \underset{\sim}{\sim} \widetilde{G}$ through a $S^{1}$-homotopy $H_{\tau}$ which extends $h_{\tau}$. Thus, for any $\widetilde{F}$ one has an extension $\widetilde{G}$ of $G$ such that $\operatorname{deg}_{K}(F, \widetilde{F})=\operatorname{deg}_{K}(G, \widetilde{G})$ (restrict the homotopy $H_{\tau}$ to the set

$$
\partial\left(I \times B_{0} \times\left(B \cap\left\{0 \leq Z_{h_{c}} \leq R, Z_{h_{c+1}}=\cdots=Z_{m}=0\right\}\right)\right) .
$$

By reversing the roles of $F$ and $G$ one has assertion (a).

(b) Let $\widetilde{F}$ be a $S^{1}$-extension to $I \times B_{0} \times\left(B \cap\left\{Z_{h_{c}}=\cdots=Z_{m}=0\right\}\right)$ of the restriction of $F$ to $\partial\left(I \times B_{0} \times\left(B \cap\left\{Z_{h_{c}}=\cdots=Z_{m}=0\right\}\right)\right.$. Thus $F \stackrel{S^{1}}{\sim}$ $(1,0,0)$ on $\partial\left(I \times B_{0} \times\left(B \cap\left\{Z_{h_{c}}=\cdots=Z_{m} \stackrel{h_{c}}{=} 0\right\}\right)\right.$ since we can deform $F$ via $\widetilde{F}(\tau t, \tau x, \tau Z)$ to $\left(\tilde{f}_{0}, \widetilde{\Phi}_{0}, 0\right)(0,0)$ and then to $(1,0,0)$ (see A). Hence, by the equivariant Borsuk extension theorem, the map $(1,0,0)$ has a $S^{1}$-equivariant extension to $\partial\left(I \times B_{0} \times B\right)$ with the same set of possible degrees as $F$.

Note that for the map $(1,0,0)$ one may choose any element in $\pi_{k+1}\left(S^{l}\right)$ (= the group of homotopy classes of maps from $\left(I \times B_{0}, \partial\left(I \times B_{0}\right)\right)$ into $(\mathbf{R} \times$ $\left.\mathbf{R}^{\prime} \backslash\{0\},(1,0)\right)$ as the first extension $\left(\tilde{f}_{0}, \widetilde{\Phi}_{0}\right)$. Similarly, one may choose as $\widetilde{F}$ (since $F=(1,0,0)$ on $\partial\left(I \times B_{0} \times\left(B \cap\left\{Z_{h_{c}}=\cdots=Z_{m}=0\right\}\right)\right)$ any element of $\pi_{k+1+2\left(h_{c}-1\right),}^{S^{1}}\left(S^{l+2 n}\right)$, regarded as the set of $S^{1}$-homotopy classes of maps from $I \times B_{0} \times\left(B \cap\left\{Z_{h_{c}}=\cdots=Z_{m}=0\right\}\right)$ into $\mathbf{R} \times \mathbf{R}^{l} \times \mathbf{C}^{n} \backslash\{0\}$ with value $(1,0,0)$ on the boundary (see Remark D.4). Since the map $F$ is defined for $0<Z_{h_{c}} \leq$ $R$, the possible degrees may differ if $\chi_{*}([\tilde{F}]) \neq \chi_{*}([\tilde{G}])$ for two equivariant extensions $\widetilde{F}$ and $\widetilde{G}$ of $(1,0,0)$ to the set $I \times B_{0} \times\left(B \cap\left\{Z_{h_{c}}=\cdots=Z_{m}=0\right\}\right)$ (note that one needs only the ordinary homotopy).

(c) Since the addition is defined on the $t$ variable only, the result follows from (b) adding the extensions.

(d) If the set of degrees reduces to a single element, then $\operatorname{deg}_{K}([0],[\tilde{0}])=$ $\operatorname{deg}_{K}((1,0,0))=0$. Conversely, if $\operatorname{deg}_{K}([0],[\tilde{0}])=0$ then the map defined as

$$
\begin{cases}\widetilde{F} & \text { on } I \times B_{0} \times\left(B \cap\left\{Z_{h_{c}}=\cdots=Z_{m}=0\right\}\right), \\ (1,0,0) & \text { for } 0<Z_{h_{c}} \leq R\end{cases}
$$


is trivial, that is, $\chi_{*}([\tilde{F}])=0$ (following Remark D.4, take $y=Z_{h_{c}} / R$ ). One may then extend $F$ to $Z_{h_{c}}=\cdots=Z_{m}=0$ as $(1,0,0)$ and get a unique degree. Q.E.D.

Remark D.4. Let $C=I \times B_{0} \times\left(B \cap\left\{Z_{h_{c}}=\cdots=Z_{m}=0\right\}\right)$ and let $\partial C$ be its boundary. Then the group of $S^{1}$-homotopy classes of equivariant maps

$$
F:(C, \partial C) \rightarrow\left(\mathbf{R} \times \mathbf{R}^{l} \times \mathbf{C}^{n} \backslash\{0\},(1,0,0)\right)
$$

is isomorphic to $\pi_{k+1+2\left(h_{c}-1\right)}^{S^{1}},\left(S^{l+2 n}\right)$. Indeed, given an equivariant map

$$
F:(C, \partial C) \rightarrow\left(\mathbf{R} \times \mathbf{R}^{l} \times \mathbf{C}^{n} \backslash\{0\},(1,0,0)\right)
$$

we define an element of $\pi_{k+1+2\left(h_{c}-1\right),}^{S^{1}}\left(S^{l+2 n}\right)$ in the following way: take $y \in$ $I=[0,1]$, with trivial action, as a new variable and then define on $\partial(C \times I)$ an equivariant map as follows:

$$
\begin{cases}F & \text { for } y=0, \\ (1,0,0) & \text { on the rest of the boundary. }\end{cases}
$$

Conversely, given a $S^{1}$-map $F: \partial(C \times I) \rightarrow \mathbf{R} \times \mathbf{R}^{l} \times \mathbf{C}^{n} \backslash\{0\}$, i.e., a representative of an element in $\pi_{k+1+2\left(h_{c}-1\right)}^{S^{1}} .\left(S^{l+2 n}\right)$, we may construct a $S^{1}$-map, in the same equivariant homotopy class, with value $(1,0,0)$ on the complement of $C \times\{0\}$. In fact, we may assume that $F=(1,0,0)$, if $y=1$ (for $y=1$, one may first deform $F$ to $F(0,0,1)$, then to $(1,0,0)$, and finally use the equivariant Borsuk extension theorem). Now on the complement of $C \times\{0\}$ the deformation $F\left(t, x_{0}, Z, \tau y+(1-\tau)\right), Z=\left(Z_{1}, \ldots, Z_{h_{c}-1}\right)$, is admissible. Then, the equivariant Borsuk extension theorem gives the result. Finally, since the compositions of the two processes are the identity, the two groups are isomorphic.

Proof of Theorem 4.6 in the cases (i) $h_{c}=1$ and (ii) $h_{c}>1,|k-l|$ odd.

(i) Let $h_{c}=1, k-l \geq 2 n-1$. In this case $\widetilde{F}=\left(\tilde{f}_{0}, \widetilde{\Phi}_{0}\right)$ is any element of $\pi_{k+1}\left(S^{l}\right)$. If $n>0$, then $\chi_{*}([\widetilde{F}])=0$ (by deformation through the $2 n$ equations).

If $n=0, k \geq l-1$ is the only nontrivial case. Then $\chi_{*}$ is an isomorphism and any possible degree is realized by the trivial map. Thus, using the equality

$$
\begin{gathered}
\left\{\operatorname{deg}_{K}([F]+[0],[\widetilde{F}]+[\widetilde{0}])\right\}=\left\{\operatorname{deg}_{K}([F],[\widetilde{F}])\right\} \\
=\left\{\operatorname{deg}_{K}([F],[\widetilde{F}])\right\}+\left\{\operatorname{deg}_{K}([0],[\widetilde{0}])\right\},
\end{gathered}
$$

one may always choose $\widetilde{F}$ in such a way that $F$ is extendable to $Z_{h_{c}+1}=\cdots=$ $Z_{m}=0$.

(ii) Let $h_{c}>1$ and $|k-l|$ odd. If $\hat{m}=h_{c}-1=n-(k+1-l) / 2$ and $\hat{h}_{c}=n+1-(k+1-l) / 2$, being the critical level for $k+1+2 \hat{m}$, then $1 \leq \hat{m}<\hat{h}_{c}$. From Theorem 4.4, we have $\chi_{*}([\widetilde{F}])=0$, except for $k+1=l$ and $\hat{m}=n$, where $\chi_{*}([\tilde{F}])=\left(N / m_{0}^{n}\right) \operatorname{deg}\left(\tilde{f}_{0}, \widetilde{\Phi}_{0}\right)$. 
Thus any degree is realized if $N=m_{0}^{n}$ and, as above, one has an extension for $F$, while if $N>m_{0}^{n}$ only the multiples of $N / m_{0}^{n}$ are realized. In fact, if $\left(\tilde{f}_{0}, \widetilde{\Phi}_{0}\right)$ is any extension of $(1,0)$ to $I \times B_{0}$ (normalized to 1 ) of degree $d$, then the map

$$
\frac{R-\|Z\|}{R}\left(\tilde{f}_{0}-1, \widetilde{\Phi}_{0}, \ldots, Z_{j}^{n_{j} / m_{0}}\left(R_{0}-\left\|x_{0}\right\|\right) t(1-t), \ldots\right)+(1,0,0)
$$

is an equivariant extension of $(1,0,0)$, with zeros only at $Z_{j}=0, j=$ $1, \ldots, n, \Phi_{0}=0, \tilde{f}_{0}=-1$, and $\left|Z_{n+1}\right|^{2}+\cdots+\left|Z_{m}\right|^{2}=R^{2} / 4$. For $h_{c}=n+1$, the $K$-degree of this map is $d N / m_{0}^{n}$ (see Theorem 4.4). On the other hand, for $d>0$, consider the equivariant map:

$$
\begin{gathered}
\left(1-\frac{2}{R}\left|Z_{n+1}\right|,\left|Z_{n+1}\right|(2 t-1),\left|Z_{n+1}\right| x_{0}, Z_{n+1}^{n_{1} / m_{0}} \bar{Z}_{n+1}^{d} Z_{1}^{d},\right. \\
\left.Z_{n+1}^{n_{2} / m_{0}} \bar{Z}_{n+1} Z_{2}, \ldots, Z_{n+1}^{n_{n} / m_{0}} \bar{Z}_{n+1} Z_{n}\right) .
\end{gathered}
$$

For $Z_{n+1}=0$, the above map coincides with the map $(1,0,0)$. Furthermore, it has only one zero at $Z_{1}=\cdots=Z_{n}=0, t=\frac{1}{2}, x_{0}=0,\left|Z_{n+1}\right|=R / 2$, and for $0 \leq Z_{n+1} \leq R$, one may deform $Z_{n+1}$ linearly to 1 in all of the components excepts the first. By the product formula of the degree, this map has degree $(-1)^{k+2} d$. Clearly, changing $2 t-1$ into $1-2 t$, one generates all the integers. Q.E.D.

We would like to summarize what we have proved if $n \geq 1$. Namely,

$$
\pi_{l-1+2(n+1),}^{S^{1}}\left(S^{l+2 n}\right) \cong \mathbf{Z}_{N / m_{0}^{n}}
$$

where the latter group is $\operatorname{ker} p_{*}$. For $|k-l|$ even and $h_{c}>1$ one has $\mid k+$ $1-l \mid$ odd and $\hat{m}=h_{c}-1=n-(k-l) / 2=\hat{h}_{c}$. Here we need to compute $\chi_{*}\left(\pi_{k+1+2 \hat{m}, \cdot}^{S^{1}}\left(S^{l+2 n}\right)\right)$ when $\hat{m}=\hat{h}_{c} \geq 1 \quad\left(\hat{h}_{c}=1\right.$ only if $h_{c}=2$, thus $k-l=$ $2 n-2)$.

Proof of Corollary 4.7. (i) If $h_{c}=1$ and $n=0$, then $\operatorname{ker} p_{*}=\{0\}$ since any degree is realized by the trivial map. On the other hand, the map $\left(f_{0}\left(t, x_{0}, Z\right)\right.$, $\left.\Phi_{0}\left(t, x_{0}, Z\right)\right)$ being nonzero on $\partial\left(I \times B_{0} \times B\right)$, one may deform $p_{*}\left([F]_{S^{1}}\right)$ to $\left(f_{0}(0,0, R), \Phi_{0}(0,0, R)\right)$ (note that $\left.m \geq 1=h_{c}\right)$. Hence $p_{*}\left([F]_{S^{1}}\right)=0$.

(We add in passing that from here one may prove directly that any invariant map from $S^{k+2}$ into $S^{l}$ is trivial. Indeed, one may assume that $f=(1,0)$ on $\partial\left(I \times B_{0}\right)$ and since the map $f$ depends only on $|Z|(m=1)$ the deformation $f\left(t, x_{0}, \tau|Z|+(1-\tau) R\right)$ is valid. $)$

If $h_{c}=1$ and $n \geq 1$, then from Lemma 4.3 the morphism $p_{*}$ is onto. Now, given any element in $\pi_{k+1}\left(S^{l+2 n}\right)$ one may represent it as $\widehat{F}=\left(f_{0}, \Phi_{0}, \Phi\right)\left(t, x_{0}\right)$ with $\widehat{F}$ of norm 1 and value $(1,0,0)$ on $\partial\left(I \times B_{0}\right)$. Consider then the equivariant map

$$
F\left(t, x_{0}, Z_{1}\right)=\left(\frac{\left|Z_{1}\right|}{R}\left(f_{0}-1\right)+1,\left|Z_{1}\right| \Phi_{0}, \ldots, Z_{1}^{n_{j} / m_{0}} \Phi_{j}, \ldots\right) .
$$


Clearly, $p_{*}\left([F]_{S^{1}}\right)=0$ and $\operatorname{deg}_{K}(F)=[\widehat{F}]$ (using $y=\left|Z_{1}\right| / R$ ). To get the isomorphism, it is enough to remark that $\operatorname{deg}_{K}$ is one to one on $\operatorname{ker} p_{*}$ when it is well defined.

(ii) For $n=0$, the assertion is clear. Suppose then that $n \geq 1$. The morphism $\chi_{*}$ on $\pi_{k}\left(S^{l}\right)$ is such that

$$
\chi_{*}\left(\left[f_{0}, \Phi_{0}, Z_{1}^{n_{1} / m_{0}}, 0, \ldots, 0\right]\right)= \begin{cases}0 & \text { if } n>1, \\ \frac{n_{1}}{m_{0}} \Sigma^{2}\left[\left(f_{0}, \Phi_{0}\right)\right], & \text { if } n=1,\end{cases}
$$

since the composition of the map (of degree $\left.n_{1} / m_{0}\right)$ defined as $\left(t, x_{0}, Z_{1}\right) \mapsto$ $\left(t, x_{0}, Z_{1}^{n_{1} / m_{0}}\right)$ with $\chi_{*}\left(\left[f_{0}, \Phi_{0}, Z_{1}\right]\right)$ induces the product by $n_{1} / m_{0}$ (see [W, p. 479]). Since

$$
\pi_{l+1}\left(S^{l}\right)= \begin{cases}0 & \text { if } l<2, \\ \mathbf{Z} & \text { if } l=2, \\ \mathbf{Z}_{2} & \text { if } l>2,\end{cases}
$$

one has the first result (the case $k>l+1$ is not needed here).

On the other hand, if $F$ has $\operatorname{deg}_{K}(f)=[\tilde{f}]$, as above, one may perform the following nonequivariant deformations: replace $\left|Z_{1}\right|$ by $\tau\left|Z_{1}\right|+(1-\tau) R$ and $\left(Z_{1}^{\alpha} \Phi_{i}, Z_{1}^{\beta} \Phi_{j}\right)$ by

$$
\left(\begin{array}{cc}
\tau & -(1-\tau) \\
(1-\tau) & \tau
\end{array}\right)\left(\begin{array}{cc}
\tau Z_{1}^{\alpha} & 1-\tau \\
(\tau-1) Z_{1}^{\alpha+\beta} & \tau Z_{1}^{\beta}
\end{array}\right)\left(\begin{array}{c}
\Phi_{i} \\
\Phi_{j}
\end{array}\right)
$$

repeatedly on $\partial\left(I \times B_{0} \times B\right), i \neq j$ from 1 to $n$. Thus,

$$
\begin{aligned}
\chi_{*}([F]) & =\left[\left(f_{0}, \Phi_{0}, Z_{1}^{\widetilde{N} / m_{0}} \Phi_{1}, \Phi_{2}, \ldots, \Phi_{n}\right)\right] \\
& \left.=\frac{\tilde{N}}{m_{0}}\left[f_{0}, \Phi_{0}, Z_{1} \Phi_{1}, \Phi_{2}, \ldots, \Phi_{n}\right)\right]
\end{aligned}
$$

(see $\left[\right.$ W, p. 479]). It is clear that if $[\widehat{F}]=\left[\widehat{F}_{1}\right]+\left[\widehat{F}_{2}\right]$, then $\chi_{*}([F])=\chi_{*}\left(\left[F_{1}\right]\right)+$ $\chi_{*}\left(\left[F_{2}\right]\right)$.

Now, if $k-l=2 n-1$, then the map $\widehat{F}:=g /\|g\|$ where

$$
\begin{aligned}
& g\left(t, x_{0}\right)=\left(2\left(\frac{\left\|x_{0}\right\|^{2}}{R_{0}^{2}}+(2 t-1)^{2}\right)-1,\right. \\
& \left.\quad(2 t-1) t(1-t)\left(R_{0}^{2}-\left\|x_{0}\right\|^{2}\right), x_{0} t(1-t)\left(R_{0}^{2}-\left\|x_{0}\right\|^{2}\right)\right)
\end{aligned}
$$

has degree 1 . In fact, consider on $\partial\left([0,1] \times I \times B_{0}\right)$ the map $\left((1-y)\left(f_{0}-1\right)+\right.$ $\left.1,(1-y) \Phi_{0},(1-y) \Phi_{1}\right)$. In the last two terms, deform both factors $(1-y)$ and $t(1-t)\left(R_{0}^{2}-\left\|x_{0}\right\|^{2}\right) /\|g\|$ to 1 . If $x_{0}=0$ and $t=\frac{1}{2}$, then $f_{0}=-1$, the first term is $2 y-1$, and one may deform it linearly to $y-1$, obtaining a map of degree 1.

Now, since $k \geq l+1$ write $\left(2 t-1, x_{0}\right):=\tilde{x}_{0} \oplus Z, Z \in \mathbf{C}$. Then $\chi_{*}([F])=$ $\left[2\left(\left|x_{0}\right|^{2} / R_{0}^{2}+(2 t-1)^{2}\right)-1, \tilde{x}_{0}, Z_{1} Z\right]$ using the same deformation of positive 
factors as above. Thus, deforming the first term linearly to $R^{2}-2\left|Z_{1}\right|^{2}$ (look at its sign on $\partial\left(I \times B_{0} \times B\right)$ if $\left.\left(\tilde{x}_{0}, Z_{1} Z\right)=(0,0)\right)$ one gets the $(k-1)$ st suspension of the Hopf map. This proves (ii).

(iii) One has $2 h_{c}=2 n-(k-l-1)$; thus if $k<l+1$ then $p_{*}=0 \quad(|k-l|$ odd), while if $k \geq l+1$ then $h_{c} \leq n$ and $p_{*}$ is onto. Furthermore, whenever $\operatorname{deg}_{K}$ is well defined and $\operatorname{deg}_{K}(F)=0$ then $[F]=0$.

In the case that $k \leq l-1$ and $n \geq 0$, consider the equivariant map

$$
\begin{aligned}
F=\left(1-\frac{2}{R}\left|Z_{h_{c}}\right|,(2 t-1)\left|Z_{h_{c}}\right|, x_{0}\left|Z_{h_{c}}\right|, \bar{Z}_{h_{c}}^{d} Z_{n+1}^{d}, \ldots, \bar{Z}_{h_{c}} Z_{h_{c}-1}, \ldots,\right. \\
\left.Z_{h_{c}}^{n_{1} / m_{0}} \bar{Z}_{h_{c}} Z_{1}, Z_{h_{c}}^{n_{2} / m_{0}} \bar{Z}_{h_{c}} Z_{2}, \ldots, \bar{Z}_{h_{c}}^{n_{n} / m_{0}} \bar{Z}_{h_{c}} Z_{n}\right) .
\end{aligned}
$$

Then $\operatorname{deg}_{K}(F)=(-1)^{k} d$ and, changing $2 t-1$ to $1-2 t$, all the integers are obtained.

If $k \geq l+1 \geq 2$, writing $x_{0}=\left(\tilde{x}_{0}, x_{1}, y_{1}, \ldots, x_{n-h_{c}+1}, y_{n-h_{c}+1}\right)$, with $\tilde{x}_{0} \in \mathbf{R}^{l-1}$, consider the equivariant map

$$
\begin{aligned}
F= & \left(1-\frac{2}{R}\left|Z_{h_{c}}\right|,(2 t-1)\left|Z_{h_{c}}\right|, \tilde{x}_{0}\left|Z_{h_{c}}\right|, Z_{h_{c}}^{n_{1} / m_{0}} \bar{Z}_{h_{c}}^{d} Z_{1}^{d}, Z_{h_{c}}^{n_{2} / m_{0}} \bar{Z}_{h_{c}} Z_{2}, \ldots,\right. \\
& \left.Z_{h_{c}}^{\left(n_{h_{c}-1}\right) / m_{0}} \bar{Z}_{h_{c}} Z_{h_{c}-1}, Z_{h_{c}}^{n_{h_{c}} / m_{0}}\left(x_{1}+i y_{1}\right), \ldots, Z_{h_{c}}^{n_{h_{c}} / m_{0}}\left(x_{n-h_{c}+1}+i y_{n-h_{c}+1}\right)\right) .
\end{aligned}
$$

Then $\operatorname{deg}_{K}(F)=(-1)^{k} d$ and, changing $2 t-1$ to $1-2 t$, all the integers are again obtained.

Hence, if $k \neq l-1$, then $\operatorname{deg}_{K}$ is an isomorphism from $\operatorname{ker} p_{*}$ onto the integers. Furthermore, by performing rotations $\left(Z_{h_{c}}^{\alpha} Z_{i}, Z_{h_{c}}^{\beta} Z_{j}\right)$ of the type considered above we have that

$$
\chi_{*}([F])=\Sigma^{k+1+2\left(h_{c}-2\right)}\left[1-(2 / R) \mid Z_{h_{c}},, Z_{h_{c}}^{\widetilde{N} / m_{0}} Z_{h_{c}}^{d+h_{c}-2} Z_{1}^{d}\right] .
$$

Since $h_{c} \geq 2$ and $k \geq 0$, one is in the stable range and

$$
\chi_{*}([F])=d\left(\tilde{N} / m_{0}+d+h_{c}\right) \Sigma^{l+2 n} \eta,
$$

where $\eta$ is the Hopf map (by composition with $Z_{1}^{d}$ one gets the first factor; in the second factor only the parity is important). Thus, $\chi_{*}([F])=0$ if $d$ is even or if $d$ is odd and $\tilde{N} / m_{0}+h_{c}$ is even. Note that if $k=l-1$ and $d$ is a multiple of $N / m_{0}^{n}$ then, if moreover $N / m_{0}^{n}$ is odd, one has that $n_{j} / m_{0}$ is odd for all $j$ 's and $\tilde{N} / m_{0}+h_{c}=\tilde{N} / m_{0}+n+1$ is odd, too. Hence $\chi_{*}([F])=0$ for the extensions of the trivial map. Thus $\chi_{*}$ is well defined on $\operatorname{ker} p_{*} \cong \mathbf{Z}_{N / m_{0}^{n}}$.

If $l=0$ and $k$ is odd, set $x_{0}=\left(y_{0}, \tilde{x}_{0}\right)$ with $\tilde{x}_{0} \in \mathbf{R}^{k-1}$, of the form $\tilde{x}_{0}=\left(x_{1}, y_{1}, \ldots, x_{(k-1) / 2}, y_{(k-1) / 2}\right)$ and let $\varphi\left(x_{0}, Z\right)$ be an invariant Urysohn function with value 0 in an invariant neighborhood of $x_{0}=0$ and $Z=$ $\left(0, \ldots, 0, Z_{h_{c}}\right)$ with $0<\left|Z_{h_{c}}\right|=a<R$ (take for example $a=R / 2$ ) and 
value 1 in an invariant neighborhood (disjoint from the previous one) of the set $\left\{\left\|x_{0}\right\| \leq R_{0}, Z=0\right\} \cup\left\{\left\|x_{0}\right\| \geq R_{0}\right\} \cup\{\|Z\| \geq R\}$. Let

$$
\begin{aligned}
F\left(t, x_{0}, Z\right)=(2 t & +2 \varphi\left(x_{0}, Z\right)-1,\left(R Z_{h_{c}}-a Z_{1}\right)^{n_{1} / m_{0}-1} Z_{1}, \ldots, \\
& \left(R Z_{h_{c}}-a Z_{h_{c}-1}\right)^{\left(n_{h_{c}-1}\right) / m_{0}-1} Z_{h_{c}-1}, Z_{h_{c}}^{n_{h_{c}} / m_{0}}\left(x_{1}+i y_{1}\right), \ldots, \\
& \left.Z_{h_{c}}^{\left(n_{n-1}\right) / m_{0}}\left(x_{(k-1) / 2}+i y_{(k-1) / 2}\right),\left(x_{0} \mp i\left(a-\left|Z_{h_{c}}\right|\right)\right)^{d} Z_{h_{c}}^{n_{n} / m_{0}}\right) .
\end{aligned}
$$

Since $\pi_{k}\left(S^{0}\right)=0$ for $k \geq 1$, the class of the map $F$ belongs to $\operatorname{ker} p_{*}$ and its $K$-degree is well defined. From the definition of $\varphi$, the only zero of $F$ is at $x_{0}=0, Z_{1}=\cdots=Z_{h_{c}-1}=0,\left|Z_{h_{c}}\right|=a$, and $t=\frac{1}{2}$. Now, if $0 \leq Z_{h_{c}} \leq R$, deform $Z_{h_{c}}^{n_{j} / m_{0}}$ to 1 for $j=h_{c}, \ldots, n$, the part $R Z_{h_{c}}-a Z_{j}$ first to $R Z_{h_{c}}$ and then to 1 for $j=1, \ldots, h_{c}-1$. A final deformation of $\varphi$ to 0 will give the function

$$
\left(2 t-1, Z_{1}, \ldots, Z_{h_{c}-1}, x_{1}+i y_{1}, \ldots, x_{(k-1) / 2}+i y_{(k-1) / 2},\left(x_{0} \mp i\left(a-\left|Z_{h_{c}}\right|\right)\right)^{d}\right)
$$

with degree $\pm d$.

Finally, if $f$ is such that $p_{*}([F]) \neq 0$, one has that

$$
\chi_{*}\left(\left[\left(f_{0}, \Phi_{0}, Z_{1}^{n_{1} / m_{0}}, \ldots, Z_{h_{c}}^{n_{h_{c}} / m_{0}}, 0, \ldots, 0\right)\right]\right)=0
$$

if $h_{c}<n$, that is, if $k>l+1$. Thus the only nontrivial case is for $k=l+1$. Hence $\chi_{*}([F])=\left(N / m_{0}^{n}\right) \Sigma^{2 h_{c}}\left[\left(f_{0}, \Phi_{0}\right)\right]$, which is nontrivial only if $N / m_{0}^{n}$ is odd and $l \geq 2$. Then all $n_{j} / m_{0}$ are odd and $\tilde{N} / m_{0}+h_{c}=\tilde{N} / m_{0}+n$ is even. Q.E.D.

Remark D.5. The morphism found in the proof of (ii):

$$
[\tilde{f}] \in \pi_{k+1}\left(S^{l+2 n}\right) \rightarrow\left[f_{0}, \Phi_{0}, Z_{1} \Phi_{1}, \Phi_{2}, \ldots, \Phi_{n}\right] \in \pi_{k+2}\left(S^{l+2 n}\right)
$$

is a particular case of the following construction. Let $B: S^{r} \rightarrow O(p)$ represent an element of $\pi_{r}(O(p))$ and let $\left(f_{0}, \Phi\right):\left\{x \in \mathbf{R}^{q+1}:\|x\| \leq 1\right\} \rightarrow \mathbf{R}^{p+1} \backslash\{0\}$ be an element of $\pi_{q}\left(S^{p}\right)$ such that $\left(f_{0}, \Phi\right)_{\|x\|=1}=(1,0)$. Consider the map $J_{*}: \pi_{r}(O(p)) \times \pi_{q}\left(S^{p}\right) \rightarrow \pi_{r+q}\left(S^{p}\right)$ defined by

$$
J_{*}\left(B(\lambda),\left(f_{0}, \Phi\right)(x)\right)=\left[f_{0}(x),\|\lambda\| B(\lambda /\|\lambda\|) \Phi(x)\right]
$$

on $\partial\left\{(\lambda, x) \in \mathbf{R}^{r+1} \times \mathbf{R}^{q+1}:\|\lambda\| \leq 1,\|x\| \leq 1\right\}$. If $p=q$, then $\left(f_{0}, \boldsymbol{\Phi}\right)(x)=$ $\left(2\|x\|^{2}-1, x\left(1-\|x\|^{2}\right)\right)$ has degree 1 (as follows from the previous arguments) and $J_{*}\left(B(\lambda),\left(f_{0}, \Phi_{0}\right)\right)=\left[1-2\|\lambda\|^{2},\|\lambda\| B(\lambda /\|\lambda\|) x\right]$ is the Whitehead $J$-homomorphism used in [I]. In the present case $r=1, q=k+1, p=l+2 n$, and $\lambda /\|\lambda\|$ is represented by $Z_{1}$. Since

$$
\pi_{1}(O(l+2 n)) \cong \begin{cases}\mathbf{Z}_{2} & \text { if } l+2 n>2, \\ \mathbf{Z} & \text { if } l+2 n=2,\end{cases}
$$

one has that $\chi_{*}([F])$ is of order 2 if $l+2 n>2$. In particular, $\chi_{*}([F])=0$ if $\tilde{N} / m_{0}$ is even $(l+2 n>2)$. Since we will not need this construction here, we shall not pursue its study. 
Proof of Theorem 4.6 in the case $h_{c}>1$ and $|k-l|$ even. If $\widetilde{F}$ is the extension on $Z_{h_{c}}=0$, then consider $\chi_{*}([\tilde{F}])$ in the situation treated in Corollary 4.7. Thus $\chi_{*}([\widetilde{F}])=0$, giving a well-defined $K$-degree, except if $h_{c}>2, \tilde{N} / m_{0}+\hat{h}_{c}$ even or $h_{c}=2, \tilde{N} / m_{0}$ odd, $l+2 n>2$ and $h_{c}=2, k=l=0$, and $n=1$. In the first two exceptions $\chi_{*}$ is onto and one may always extend the map to the level $Z_{h_{c}}$. The argument for the last case runs as for $k=l-1$. Q.E.D.

Proof of Corollary 4.8. Since $h_{c}=n+1-(k-l) / 2$, if $k \geq l+2$ then $h_{c} \leq n$ and $p_{*}$ is onto. If $k=l$, then $h_{c}=n+1>n$. Hence from Theorem 4.4, we obtain $p_{*}=0$.

If $\tilde{N} / m_{0}+h_{c}$ is odd (excluding the case $n=1$ and $k=l=0$ ) then $\operatorname{ker} p_{*}=0$.

If $\tilde{N} / m_{0}+h_{c}$ is even (excluding the case $n=1$ and $k=l=0$ ) then $\operatorname{deg}_{K}(F)$ is well defined.

Now, for $k \leq l-2$ and $n \geq 0$, we consider the equivariant map

$$
\begin{aligned}
\left(\left(\left|Z_{h_{c}}\right|^{2}-\left|Z_{n+1}\right|^{2}\right)^{2}-\left|Z_{n+1}\right|^{2}\left|Z_{h_{c}}\right|^{2}+(2 t-1)^{2}+(R / 2)^{4},\right. & \\
\bar{Z}_{h_{c}} Z_{n+1}\left(\left|Z_{h_{c}}\right|^{2}-\left|Z_{n+1}\right|^{2}+\right. & i(2 t-1)),\left|Z_{h_{c}}\right| x_{0}, \bar{Z}_{h_{c}} Z_{n+2}, \ldots, \\
& \left.\bar{Z}_{h_{c}} Z_{h_{c}-1}, Z_{h_{c}}^{n_{1} / m_{0}} \bar{Z}_{h_{c}} Z_{1}, \ldots, \bar{Z}_{h_{c}}^{n_{n} / m_{0}} \bar{Z}_{h_{c}} Z_{n}\right)
\end{aligned}
$$

(if $n=0$, the last terms are not present. If $n=0, k=0$, and $l=2$ then only the first two equations are present and the term $\bar{Z}_{2} Z_{1}$ may be replaced by $\bar{Z}_{2}^{d} Z_{1}^{d}$ and $2 t-1$ by $1-2 t$ respectively).

If $k \geq l$ (thus $n \geq 1$, since $h_{c}>1$ ) let

$$
x_{0}=\left(\tilde{x}_{0}, x_{1}, y_{1}, \ldots, x_{n-h_{c}+1}, y_{n-h_{c}+1}\right)
$$

with $\tilde{x}_{0} \in \mathbf{R}^{l}$. Define a map by

$$
\begin{aligned}
\left(\left(\left|Z_{h_{c}}\right|^{2}-\left|Z_{1}\right|^{2}\right)^{2}-\left|Z_{1}\right|^{2}\left|Z_{h_{c}}\right|^{2}+(2 t-1)^{2}+(R / 2)^{4},\left|Z_{h_{c}}\right| \tilde{x}_{0},\right. \\
\quad Z_{h_{c}}^{n_{1} / m_{0}} \bar{Z}_{h_{c}} Z_{1}\left(\left|Z_{h_{c}}\right|^{2}-\left|Z_{1}\right|^{2}+i(2 t-1)\right), Z_{h_{c}}^{n_{2} / m_{0}} \bar{Z}_{h_{c}} Z_{2}, \ldots, \\
\left.\quad Z_{h_{c}}^{\left(n_{h_{c}-1}\right) / m_{0}} \bar{Z}_{h_{c}} Z_{h_{c}-1}, Z_{h_{c}}^{n_{h_{c}} / m_{0}}\left(x_{1}+i y_{1}\right), \ldots, Z_{h_{c}}^{n / m_{0}}\left(x_{n-h_{c}+1}+i y_{n-h_{c}+1}\right)\right) .
\end{aligned}
$$

Note that the above two maps belong to $\operatorname{ker} p_{*}$ and their $K$-degree is the class of

$$
\begin{array}{r}
\Sigma^{k+2\left(h_{c}-2\right)}\left(\left(\left|Z_{h_{c}}\right|^{2}-\left|Z_{1}\right|^{2}\right)^{2}-\left|Z_{1}\right|^{2}\left|Z_{h_{c}}\right|^{2}+(2 t-1)^{2}+(R / 2)^{4},\right. \\
\left.Z_{1}\left(\left|Z_{h_{c}}\right|^{2}-\left|Z_{1}\right|^{2}+i(2 t-1)\right)\right)
\end{array}
$$

where $0 \leq Z_{h_{c}} \leq R$ (consider $Z_{1}^{d}$ if $n=0, k=0, l=2$ ). 
Now perform the deformation

$$
\begin{aligned}
\left(\left(\left|Z_{h_{c}}\right|^{2}-\frac{R^{2}}{4}-\tau\left(\left|Z_{1}\right|^{2}-\frac{R^{2}}{4}\right)^{2}-\left(\tau\left|Z_{h_{c}}\right|^{2}+(1-\tau) R^{2}\right)\left|Z_{1}\right|^{2}\right)\right. \\
+\tau\left(\frac{R^{2}}{4}\right)^{2}+(2 t-1)^{2}, \\
\left.\quad Z_{1}\left(\left|Z_{h_{c}}\right|^{2}-\frac{R^{2}}{4}-\tau\left(\left|Z_{1}\right|^{2}-\frac{R^{2}}{4}\right)+i(2 t-1)\right)\right) .
\end{aligned}
$$

If $Z_{1}=0$, then the first equation is positive unless $\tau=0,2 t-1=0$, and $Z_{h_{c}}=R / 2$ (which is not a point on the boundary). If $t=\frac{1}{2},\left|Z_{h_{c}}\right|^{2}-R^{2} / 4=$ $\tau\left(\left|Z_{1}\right|^{2}-R^{2} / 4\right)$, and $\left|Z_{h_{c}}\right|^{2}+\left|Z_{1}\right|^{2}=R^{2}$ then $\left|Z_{1}\right|^{2}, Z_{h_{c}}>R^{2} / 4$; thus the first equation is strictly negative. Writing the term

$$
\left|Z_{h_{c}}\right|^{2}-R^{2} / 4=\left(Z_{h_{c}}-R / 2\right)\left(\tau\left(Z_{h_{c}}+R / 2\right)+(1-\tau)\right),
$$

one gets the class of $\left(\left(Z_{h_{c}}-R / 2\right)^{2}-\left|Z_{1}\right|^{2}+(2 t-1)^{2}, Z_{1}\left(Z_{h_{c}}-R / 2+i(2 t-1)\right)\right)$, which is the Hopf map $\eta:\left\{\left(x_{1}, x_{2}\right) \in \mathbf{C}^{2}:\left|x_{1}\right|^{2}+\left|x_{2}\right|^{2} \leq 1\right\} \rightarrow S^{2}$ given by $\eta\left(x_{1}, x_{2}\right)=\left(\left|x_{1}\right|^{2}-\left|x_{2}\right|^{2}, x_{1} x_{2}\right)$. Recall that $\eta$ generates $\pi_{3}\left(S^{2}\right)$. If $n=0$, $k=0$, and $l=2$, one obtains $\pm d \eta$ by changing $2 t-1$ to $1-2 t$. Furthermore, by performing rotations on $\left(Z_{h_{c}}^{\alpha} \Phi_{i}, Z_{h_{c}}^{\beta} \Phi_{j}\right)$ we have that

$$
\begin{gathered}
\chi_{*}([F])=\Sigma^{k+2\left(h_{c}-2\right)}\left[\left(\left|Z_{h_{c}}\right|^{2}-\left|Z_{1}\right|^{2}\right)^{2}-\left|Z_{h_{c}}\right|^{2}\left|Z_{1}\right|^{2}+(2 t-1)^{2}+\tau\left(\frac{R}{2}\right)^{4},\right. \\
\left.Z_{h_{c}}^{\widetilde{N} / m_{0}} \bar{Z}_{h_{c}}^{h_{c}-1} Z_{1}\left(\left|Z_{h_{c}}\right|^{2}-\left|Z_{1}\right|^{2}+i(2 t-1)\right)\right]
\end{gathered}
$$

for $\tau=1$ (the term $\bar{Z}_{h_{c}}^{h_{c}-1} Z_{1}$ has to be replaced by $\bar{Z}_{h_{c}}^{h_{c}-2+d} Z_{1}^{d}$ if $n=0$, $k=0$, and $l=2)$. Note that the homotopy is valid. Replacing $\left|Z_{h_{c}}\right|^{2}$ by $\left|Z_{h_{c}}\right|^{2}\left(\tau+(1-\tau)\left|Z_{h_{c}}\right|^{\alpha-2}\right)$ and a similar scaling on $Z_{1}$, one has, by the composition law,

$$
\begin{aligned}
\chi_{*}([F])= & d\left(\frac{\tilde{N}}{m_{0}}-h_{c}+2-d\right) \\
& \cdot \Sigma^{k+2\left(h_{c}-2\right)}\left[\left(\left|Z_{2}\right|^{2}-\left|Z_{1}\right|^{2}\right)^{2}-\left|Z_{1}\right|^{2}\left|Z_{2}\right|^{2}+(2 t-1)^{2},\right. \\
& \left.\quad Z_{1} Z_{2}\left(\left|Z_{2}\right|^{2}-\left|Z_{1}\right|^{2}+i(2 t-1)\right)\right]
\end{aligned}
$$

which is the composition of the map $\left(Z_{1}, Z_{2}, t\right) \mapsto\left(\left|Z_{1}\right|^{2}-\left|Z_{2}\right|^{2}+\right.$ $i(2 t-1), Z_{1} Z_{2}$ ) (i.e., the suspension of the Hopf map) with the Hopf map itself. Now $\eta \circ(\Sigma \eta)$ generates $\pi_{n+2}\left(S^{n}\right) \cong \mathbf{Z}_{2}$ for $n \geq 2$. Thus $\chi_{*}([F])$ generates $\mathbf{Z}_{2}$ if and only if $d\left(\tilde{N} / m_{0}-h_{c}+2-d\right)$ is odd $(d=1$ except for $n=0$, $k=0, l=2$ ). 
Furthermore, if $p_{*}([F])=\left[f_{0}, \Phi_{0}\right] \neq 0$ (hence $k>l$ ) then

$$
\chi_{*}\left(f_{0}, \Phi_{0}, Z_{1}^{n_{1} / m_{0}}, \ldots, Z_{h_{c}}^{n_{n} / m_{0}}, 0, \ldots, 0\right)=0 \text { if } k \geq l+3
$$

since then $h_{c}<n$. If $k=l+2$, then $h_{c}=n$ and $\chi_{*}([F])=\left(N / m_{0}^{n}\right) \Sigma^{2 n}\left[f_{0}, \Phi_{0}\right]$ $=\left(N / m_{0}^{n}\right) \Sigma^{2 n} p_{*}([F])$. Since $p_{*}([F])=0$ for $l=0,1$ then $\chi_{*}([F]) \neq 0$ only if $\left(N / m_{0}\right)^{n}$ is odd: $p_{*}([F])=\eta \circ \Sigma \eta$, or a suspension, if $l \geq 2$.

It remains to study the case $n=1, k=l=0$. In this case, the equivariant map

$$
\begin{array}{r}
F:=\left(\left(\left|Z_{2}\right|^{2}-\left|Z_{1}\right|^{2}\right)^{2}-\left|Z_{2}\right|^{2}\left|Z_{1}\right|^{2}+(2 t-1)^{2}+\left(R^{2} / 4\right)^{2},\right. \\
\left.Z_{2}^{n_{1} / m_{0}} \bar{Z}_{2}^{d} Z_{1}^{d}\left(\left|Z_{2}\right|^{2}-\left|Z_{1}\right|^{2} \pm i(2 t-1)\right)\right)
\end{array}
$$

has $K$-degree $\pm d$, according to the orientations (since the trivial map has extensions with $K$-degrees which are multiples of $\tilde{N} / m_{0}$, one obtains the isomorphism). Furthermore, $\chi_{*}([F])=\left(n_{1} / m_{0}-d\right) d(\eta \circ \Sigma \eta)$; here $h_{c}=2$, and $\chi_{*}([F])=0$ if $d$ is a multiple of $n_{1} / m_{0}$. Thus, $\chi_{*}$ is well defined on $\operatorname{ker} p_{*}$. Q.E.D.

Remark D.6. If $\beta: \mathbf{R} \times \mathbf{R}^{l} \times \mathbf{C}^{n} \rightarrow \mathbf{R} \times \mathbf{R}^{l} \times \mathbf{C}^{n}$ is the map defined by

$$
\left(t, y_{0}, \xi_{1}, \ldots, \xi_{n}\right) \mapsto\left(t, y_{0}, \xi_{1}^{\nu_{1}}, \ldots, \xi_{n}^{\nu_{n}}\right),
$$

for $\nu_{i} \geq 1$, of degree $\prod \nu_{i}$, then $\operatorname{deg}_{K}(\beta F)=\prod \nu_{i} \operatorname{deg}_{K}(F)$ (see [W, Theorem 8.2]; one has the suspension in the first variable). Thus, the induced map $\beta_{*}$ on $\pi_{k+2 m}^{S^{1}},\left(S^{l+2 n}\right), m \leq h_{c}$, will be one to one (not onto) if either $m<h_{c}$ or $m=h_{c}=1$ or $m=h_{c}>1,|k-l|$ is odd, and $k \neq l-1$. In the other cases $N$ is changed into $N \prod \nu_{i}$ and $\widetilde{N}$ into $\sum \nu_{j} n_{j}$; thus these groups depend on the action in the range. Furthermore, it is easy to verify that $\chi_{*} \beta_{*}=\beta_{*} \chi_{*}$. A similar effect will be felt if $m_{0}$ is replaced by 1 or by another common divisor of the $m_{j}$ 's. In fact, if on $\left(\xi_{1}, \ldots, \xi_{m}\right)$ the standard $S^{1}$-action is given and on $\left(Z_{1}, \ldots, Z_{n}\right)$ the action $\mathrm{e}^{i m_{0} \varphi}$, then the induced homomorphism

$$
\Theta^{*}: \pi_{k+2 h_{c}}^{S^{1}}\left(S^{l+2 n}\right) \rightarrow \pi_{k+2 h_{c}}^{S^{1}},\left(S^{l+2 n}\right)
$$

is an isomorphism on $\operatorname{Im} p_{*}$ and on $\operatorname{ker} p_{*}$ one has $\Theta^{*}[F]=m_{0}^{h_{c}-1}[F]$. This can be proved either by using the composition law $\left(\xi_{1}, \ldots, \xi_{h_{c}-1}, \xi_{h_{c}}\right) \mapsto$ $\left(\xi_{1}^{m_{0}}, \ldots, \xi_{h_{c}-1}^{m_{0}}, \xi_{h_{c}}^{m_{0}}\right)$ of degree $m_{0}^{h_{c}-1}$ from the set $\left\{|\xi| \leq R, 0 \leq \xi_{h_{c}} \leq R\right\}$ onto $\{|Z| \leq R, 0 \leq Z \leq R\}$ (note that $\xi_{h_{c}}$ being real positive, $\xi_{h_{c}}$ is deformable to $\xi_{h_{c}} R^{m_{0}-1}$ linearly) or by looking directly at the generator of the group. Hence, in order to have the degree of the invariant part nontrivial, there is no gain in considering the action $\mathbf{e}^{i m_{0} \varphi}$ if $h_{c}=1$ or $|k-l|$ is odd, and if $|k-l|$ is even only if $m_{0}$ is even and $\widetilde{N} / m_{0}+h_{c}$ is even (note, however, that $\chi_{*}$ detects the nontrivial elements in $\left.\pi_{k+2 h_{c}}^{S^{1}}\left(S^{l+2 n}\right)\right)$.

Proof of Theorem 4.9. (i) If $\bar{\Omega} \cap\{Z=0\}=\varnothing$, then $2 t+2 \varphi\left(x_{0}, 0\right)-1=2 t+1$ for $x_{0}$ in any small neighborhood $N$ of $\partial \Omega$. Thus, $p_{*}([F])=\left[2 t+1, \Phi_{0}\left(x_{0}, 0\right)\right]=$ 
0 . Hence, if either $m<h_{c}$ or if $m=h_{c}=1, n=0$, or $h_{c}>1,|k-l|$ is even, $k+l+n>1$ and $\tilde{N} / m_{0}+h_{c}$ is odd then all the $S^{1}$-degrees vanish. By a rotation in $Z$ (hence equivariant) and a translation in $x_{0}$, assume that $\left(x_{0}=0\right.$, $Z=(0, \ldots, 0, a))$ belongs to $\Omega, a>0$. In the case that $\bar{\Omega} \cap\{Z=0\} \neq \varnothing$ one may assume that $\left(x_{0}, Z=(0, \ldots, 0, \varepsilon)\right)$ is in $\Omega$, for any small $\varepsilon$.

(a) Let $h_{c}=1$ and set $S^{k}=\partial\left\{x_{0} \in \mathbf{R}^{k}:\left\|x_{0}\right\| \leq R_{0}, 0 \leq Z_{1} \leq R\right\}$. Any element in $\pi_{k}\left(S^{l+2 n-1}\right)$. can be written as $\left(\Phi_{0}, \Phi\right)\left(x_{0}, Z_{1}\right)$, where $\Phi_{0}$ has values in $\mathbf{R}^{l}$ and $\Phi$ has values in $\mathbf{C}^{n}$. Furthermore, one may extend $\left(\Phi_{0}, \Phi\right)$ radially to the ball $\left\{\left(x_{0}, Z_{1}\right):\left\|x_{0}\right\| \leq R_{0}, 0 \leq Z_{1} \leq R\right\}$ in such a way that the map $\left(\Phi_{0}, \Phi\right)$ is zero only at $x_{0}, Z_{1}=a<R$. Now define the equivariant map

$$
\left(\widetilde{\Phi}_{0}, \widetilde{\Phi}\right)\left(x_{0}, Z_{1}\right)=\left(\Phi_{0}\left(x_{0},\left|Z_{1}\right|\right), Z_{1}^{n_{1} / m_{0}} \Phi\left(x_{0},\left|Z_{1}\right|\right)\right) \text {. }
$$

This map has only one zero at $x_{0}=0,\left|Z_{1}\right|=a$. If $0 \leq Z_{1} \leq R_{1}$, the class of the map $\left(2 t+2 \varphi-1, \widetilde{\Phi}_{0}, \widetilde{\Phi}\right)$ is that of $\left(2 t-1, \Phi_{0}, \Phi\right)$, i.e., the suspension of $\left(\Phi_{0}, \Phi\right)$ (indeed, first deform $\widetilde{\Phi}$ to $\Phi$ and then $2 t+2 \varphi-1$ to $2 t-1$ recalling that $\left.\varphi\left(x_{0}, 0\right)=1, \varphi(0, a)=0\right)$. Conversely, given an equivariant map $\left(\Phi_{0}, \Phi\right)$, the map $\left(2 t+2 \varphi-1, \widetilde{\Phi}_{0}, \widetilde{\Phi}\right)$ will be a suspension, for $0 \leq Z_{1} \leq R$, if $\left(\Phi_{0}, \Phi\right)\left(x_{0},\left|Z_{1}\right|\right)$ has a nonzero extension from the set $\partial \Omega \cap\left\{0 \leq Z_{1} \leq R\right\}$ to $(\bar{B} \backslash \Omega) \cap\left\{0 \leq Z_{1} \leq R\right\}$, for instance, if $\Omega=\left\{\left\|x_{0}\right\|<R_{0}, r<\left|Z_{1}\right|<R\right\}$; then for $Z_{1}=r$, the map $\left(\Phi_{0}, \Phi\right)$ is deformable to $\left(\Phi_{0}, \Phi\right)(0, r)$. Indeed, the last map is extendable to $0 \leq Z_{1} \leq r$; then the same holds for $\left(\Phi_{0}, \Phi\right)$. For $\left\|x_{0}\right\| \geq R_{0}$ or $\left|Z_{1}\right| \geq R$, one may extend the map radially without zeros.

If $\bar{\Omega} \cap\{Z=0\} \neq \varnothing$, take

$$
\left(\Phi_{0}, \Phi\right):\left(\left\{\left\|x_{0}\right\| \leq \varepsilon\right\},\left\|x_{0}\right\|=\varepsilon\right) \rightarrow\left(S^{l+2 n-1},(1,0, \ldots, 0)\right)
$$

and get the map $\left(\widetilde{\Phi}_{0}, \tilde{\Phi}\right): \partial\left\{\left\|x_{0}\right\| \leq R_{0}, 0 \leq Z_{1} \leq R\right\} \rightarrow \mathbf{R}^{l} \times \mathbf{C}^{n} \backslash\{0\}$ defined by $\left(\widetilde{\boldsymbol{\Phi}}_{0}, \widetilde{\boldsymbol{\Phi}}\right)\left(x_{0}, Z_{1}\right)=\left(Z_{1} / \varepsilon\right)\left(\left(\Phi_{0}, \Phi\right)-(1,0, \ldots, 0)\right)+(1,0, \ldots, 0)$, extending $\left(\Phi_{0}, \Phi\right)$ as $(1,0, \ldots, 0)$ for $\varepsilon \leq\left\|x_{0}\right\| \leq R_{0}$. Thus the zeros of $\left(\widetilde{\Phi}_{0}, \widetilde{\Phi}\right)$ are in $\Omega$ and one may repeat the above argument provided that $l+2 n \geq 2$ (if $l=0,1$ and $n=0$ there is nothing to prove); one obtains in this way any element in $\operatorname{ker} p_{*}$ which is a suspension.

(b) We now consider the case $h_{c}>1,|k-l|$ odd. If $k \leq l-1$ and $n \geq 0$, the map

$$
\begin{aligned}
\left(a-\left|Z_{h_{c}}\right|, x_{0}, \bar{Z}_{h_{c}}^{d} Z_{n+1}^{d}, \ldots, \bar{Z}_{h_{c}} Z_{h_{c}-1}, Z_{h_{c}}^{n_{1} / m_{0}} \bar{Z}_{h_{c}} Z_{1},\right. & \\
& \left.Z_{h_{c}}^{n_{2} / m_{0}} \bar{Z}_{h_{c}} Z_{1}, \ldots, Z_{h_{c}}^{n_{n} / m_{0}} \bar{Z}_{h_{c}} Z_{n}\right)
\end{aligned}
$$

has $K$-degree $(-1)^{k} d$ and, changing $\bar{Z}_{h_{c}}^{d} Z_{n+1}^{d}$ to $Z_{h_{c}}^{d} \bar{Z}_{n+1}^{d}$, we have that the $K$-degree is $(-1)^{k+1} d$.

If $k \geq l+1 \geq 2$, the map

$$
\begin{aligned}
& \begin{array}{l}
\left(a-\left|Z_{h_{c}}\right|, \tilde{x}_{0}, Z_{h_{c}}^{n_{1} / m_{0}} \bar{Z}_{h_{c}}^{d} Z_{1}^{d}, Z_{h_{c}}^{n_{2} / m_{0}} \bar{Z}_{h_{c}} Z_{2}, \ldots, Z_{h_{c}}^{\left(n_{h_{c}-1}\right) / m_{0}} \bar{Z}_{h_{c}} Z_{h_{c}-1},\right. \\
\left.\qquad Z_{h_{c}}^{n_{h_{c}} / m_{0}}\left(x_{1}+i y_{1}\right), \ldots, Z_{h_{c}}^{n_{h_{c}} / m_{0}}\left(x_{n-h_{c}+1}+i y_{n-h_{c}+1}\right)\right)
\end{array} \\
& \text { also has } K \text {-degree }(-1)^{k} d .
\end{aligned}
$$


If $l=0$, the map given in the proof of Corollary 4.8 already has the right form. Note that if $l \geq 1$, replacing $a$ with $\varepsilon$, one also covers the case when $\bar{\Omega} \cap\{Z=0\} \neq \varnothing$. Moreover, if $l=0$, with $\Phi\left(x_{0}, 0\right)=0$, one cannot have $\bar{\Omega} \cap\{Z=0\} \neq \varnothing$ and $\Phi\left(x_{0}, 0\right) \neq 0$ on $\partial \Omega$, unless $k=0$ also. The case $k=l=0$ will be treated below.

Let $h_{c}>1$ and $|k-l|$ even. If $1 \leq k \leq l-2$, then choose $\varepsilon$ so small that the point $x_{0}=0, Z_{i}=0$ if $i \neq h_{c}, n+1, Z_{h_{c}}, Z_{n+1}$ with $\left|Z_{h_{c}}\right|=a$, $\left|Z_{n+1}\right|=\varepsilon$, belongs to $\Omega$. Write $x_{0}=\left(y_{0}, \tilde{x}_{0}\right)$ and consider the map

$$
\begin{aligned}
& \left(\left(\varepsilon^{2}\left|Z_{h_{c}}\right|^{2}-a^{2}\left|Z_{n+1}\right|^{2}\right)^{2}-\frac{a^{2}}{\varepsilon^{2}}\left|Z_{n+1}\right|^{2}\left|Z_{h_{c}}\right|^{2}+y_{0}^{2}+a^{4},\right. \\
& \bar{Z}_{h_{c}} Z_{n+1}\left(\varepsilon^{2}\left|Z_{h_{c}}\right|^{2}-a^{2}\left|Z_{n+1}\right|^{2}+i y_{0}\right), \tilde{x}_{0}, \\
& \left.\bar{Z}_{h_{c}} Z_{n+2}, \ldots, \bar{Z}_{h_{c}} Z_{h_{c}-1}, Z_{h_{c}}^{n_{1} / m_{0}} \bar{Z}_{h_{c}} Z_{1}, \ldots, Z_{h_{c}}^{n_{n} / m_{0}} \bar{Z}_{h_{c}} Z_{n}\right) .
\end{aligned}
$$

The zeros of the above map are in $\Omega$ and one again gets a suspension by deforming $\varphi$ to 0 . The coefficients $\varepsilon$ and $a$ can then be replaced by 1 . The case $\bar{\Omega} \cap\{Z=0\} \neq \varnothing$ can be treated in a similar way by taking $\varepsilon=a$.

If $1 \leq l \leq k$, choose $\varepsilon$ as before (i.e., such that $x_{0}=(0, \varepsilon, 0, \ldots, a)$ is in $\Omega)$. Writing $\tilde{x}_{0}=\left(x_{0}, v_{0}\right)$, the map

$$
\begin{aligned}
& \left(\left(\varepsilon^{2}\left|Z_{h_{c}}\right|^{2}-a^{2}\left|Z_{1}\right|^{2}\right)^{2}-\frac{a^{2}}{\varepsilon^{2}}\left|Z_{1}\right|^{2}\left|Z_{h_{c}}\right|^{2}+x_{0}^{2}+a^{4}, v_{0},\right. \\
& Z_{h_{c}}^{n_{1} / m_{0}} \bar{Z}_{h_{c}} Z_{1}\left(\left(\varepsilon\left|Z_{h_{c}}\right|\right)^{2}-\left(a\left|Z_{1}\right|\right)^{2}+i x_{0}\right), Z_{h_{c}}^{n_{2} / m_{0}} \bar{Z}_{h_{c}} Z_{2}, \ldots, \\
& \left.\quad Z_{h_{c}}^{n_{h_{c}-1} / m_{0}} \bar{Z}_{h_{c}} Z_{h_{c}-1}, Z_{h_{c}}^{n_{h_{c}} / m_{0}}\left(x_{1}+i y_{1}\right), \ldots, Z_{h_{c}}^{n_{n} / m_{0}}\left(x_{n-h_{c}+1}+i y_{n-h_{c}+1}\right)\right)
\end{aligned}
$$

has a nonzero $K$-degree. The case $\bar{\Omega} \cap\{Z=0\} \neq \varnothing$ can be treated in a similar way by choosing $\varepsilon=a$.

If $k=0, l \geq 2, l+2 n>2$, then the map

$$
\begin{aligned}
\left(\left|Z_{h_{c}}\right|-a,\left|Z_{1}\right|-\varepsilon, \bar{Z}_{h_{c}} Z_{3}, \ldots, \bar{Z}_{h_{c}} Z_{l / 2+1}, Z_{h_{c}}^{n_{1} / m_{0}} \bar{Z}_{1} Z_{2},\right. \\
\left.Z_{h_{c}}^{n_{2} / m_{0}} \bar{Z}_{h_{c}} Z_{l / 2+2}, \ldots, Z_{h_{c}}^{n_{n} / m_{0}} \bar{Z}_{h_{c}} Z_{h_{c}-1}\right)
\end{aligned}
$$

has $K$-degree which is the $(l+2 n-2)$-suspension of the map $\left(\left|Z_{1}\right|-\varepsilon, \bar{Z}_{1} Z_{2}\right)$, that is, the Hopf map $(\varepsilon=a$ if $\bar{\Omega} \cap\{Z=0\} \neq \varnothing)$.

If $k=0, l=2, n=0$, then $h_{c}=2$ and via a rotation one may assume that $\bar{\Omega} \cap\left\{Z_{2}=0\right\} \neq \varnothing$. Then $\Phi_{0}\left(Z_{1}, 0\right)=\Phi_{0}\left(\left|Z_{1}\right|, 0\right) \neq 0$ on $\partial \Omega$. Since $l=2$, the restriction of $\Phi_{0}\left(\left|Z_{1}\right|, 0\right)$ to $\partial \Omega$ extends without zeros to the set $0 \leq\left|Z_{1}\right| \leq R$. Moreover, if the restrictions to $\partial \Omega$ of $\Phi_{0}\left(Z_{1},\left|Z_{2}\right|\right)$ and of $\Phi_{0}\left(\left|Z_{1}\right|, 0\right)$ have a nonzero extension to the two-sphere: $\partial\left\{\|Z\| \leq R, 0 \leq Z_{2} \leq R\right\}$, then the $K$-degree of the above map is the suspension of a map from $S^{2}$ into $\mathbf{R}^{2} \backslash\{0\}$, 
which is always trivial. This is the case if either $\Omega=\{r<|Z|<R\}$ or $\Omega=\{|Z|<R\}$ (in this case $0 \in \Omega$ ).

If $l=0, k \geq 4$ (then one is restricted to the case $\bar{\Omega} \cap\{Z=0\}=\varnothing$ since $\left.\Phi_{0}\left(x_{0}, 0\right)=0\right)$, write $x_{0}=\left(x_{1}, \ldots, x_{k / 2}\right)$, with $x_{j}$ complex, and consider the map

$$
\begin{aligned}
&\left(Z _ { h _ { c } } ^ { n _ { 1 } / m _ { 0 } } \left(\left|Z_{h_{c}}\right|-\right.\right.\left.a+i\left(\left|x_{1}\right|^{2}-\left|x_{2}\right|^{2}\right)\right), Z_{h_{c}}^{n_{2} / m_{0}} x_{1} x_{2}, Z_{h_{c}}^{n_{3} / m_{0}} x_{3}, \ldots, Z_{h_{c}}^{n_{k / 2} / m_{0}} x_{k / 2}, \\
&\left.\left(R Z_{h_{c}}-a Z_{1}\right)^{n_{k / 2+1} / m_{0}-1} Z_{1}, \ldots,\left(R Z_{h_{c}}-a Z_{h_{c}-1}\right)^{n_{n} / m_{0}-1} Z_{h_{c}-1}\right) .
\end{aligned}
$$

The zeros of this map are either if $Z=0$, with $\varphi\left(x_{0}, 0\right)=1$, or $\left|Z_{h_{c}}\right|=a$, $x_{0}=0, Z_{i}=0, i=1, \ldots, h_{c}-1$ with $\varphi=0$, or if $\left|Z_{h_{c}}\right|=a, x_{0}=0,\left|Z_{i}\right|=R$ for some $i$ with $\varphi=1$. To compute the $K$-degree of this map, one may deform the factor $Z_{h_{c}}^{n_{i} / m_{0}}$ to 1 (for $j=1, \ldots, n_{k / 2}$ ) and the factor $R Z_{h_{c}}-a Z_{j}$ to $R Z_{h_{c}}$ and then to 1 , since on these zeros $\varphi=1$. Moreover, since the zeros of the $\operatorname{map}\left(\left|Z_{h_{c}}\right|-a+i\left(\left|x_{1}\right|^{2}-\left|x_{2}\right|^{2}\right), x_{1} x_{2}, x_{3}, \ldots, x_{k / 2}, Z_{1}, \ldots, Z_{h_{c}-1}\right)$ are in $\Omega$, one may deform $\varphi$ to 0 and obtain the $(2 n-2)$-suspension of the Hopf map. Since $n \geq 3$, this generates the only nontrivial degree.

If $l=0, k=2$, consider the map

$$
\begin{aligned}
\left(Z_{h_{c}}^{n_{1} / m_{0}}\left(\left|Z_{h_{c}}\right|-a+i\left(\left|x_{1}\right|^{2}-\left|Z_{1}\right|^{2}\right)\right), Z_{1}\left(R Z_{h_{c}}-a Z_{1}\right)^{n_{2} / m_{0}-1}\left(x_{1}\left|Z_{h_{c}}\right|-a \varepsilon\right)\right. \\
\left.Z_{2}\left(R Z_{h_{c}}-a Z_{2}\right)^{n_{3} / m_{0}-1}, \ldots, Z_{n-1}\left(R Z_{h_{c}}-a Z_{n-1}\right)^{n_{n} / m_{0}-1}\right)
\end{aligned}
$$

where $\varepsilon$ is so small that $\left(Z=(\varepsilon, 0, \ldots, 0, a), x_{0}=(\varepsilon, 0)\right)$ belongs to $\Omega$. Clearly the zeros of this map are either $Z=0$ with $\varphi\left(x_{0}, 0\right)=1$, or at $\left|Z_{h_{c}}\right|=$ $a, Z_{i}=0, x_{0}=0$ with $\varphi=0$, or at $\left|Z_{h_{c}}\right|=a, Z_{i}=0, i \geq 2,\left|Z_{1}\right|=$ $x_{1}=\varepsilon$ with $\varphi=0$, and $\left|Z_{h_{c}}\right|=a,\left|Z_{i}\right| \stackrel{=}{=} R$ for some $i$, with $\varphi=1$. To compute its $K$-degree, one may deform $Z_{h_{c}}^{n_{1} / m_{0}}$ to $1, R Z_{h_{c}}-a Z_{i}$ first to $R Z_{h_{c}}$ and then to $1, x_{1}\left|Z_{h_{c}}\right|-a \varepsilon$ to $x_{1} a$ and then to $x_{1}$. Note that the map $\left(Z_{h_{c}}-a+i\left(\left|x_{1}\right|^{2}-\left|Z_{1}\right|^{2}\right), Z_{1} x_{1}, Z_{2}, \ldots, Z_{n-1}\right)$ has its zeros only in $\Omega$. Thus $\varphi$ may be deformed to 0 and hence one obtains a $(2 n-2)$-suspension of the Hopf map. Note that this argument can be extended to the case $k \geq 4$.

If $l=0, k=0$, then $h_{c}=n+1$ and $p_{*}\left([F]_{S^{1}}\right)=0$ (see Theorem 4.4). If $0 \in \bar{\Omega}$, then $p_{*}\left([F]_{S^{1}}\right)=\operatorname{deg}(2 t-1)=1$. Thus, once again we cannot have $0 \in \Omega$. In fact, the set $B \backslash \bar{\Omega}$ is connected: if not, let $\Omega_{1}$ be the connected component of 0 , the set $\Omega_{1}$ would be bounded, invariant, and $\Phi_{0} \neq 0$ on $\partial \Omega_{1}$. Then it follows from Theorem 4.4 that $\operatorname{deg}\left(\Phi\left(Z_{1}, \ldots, Z_{n}, 0\right), \Omega_{1} \cap\left\{Z_{n+1}=\right.\right.$ $0\}, 0)=\left(N / m_{0}^{n}\right) \operatorname{deg}(2 t-1) \neq 0$. But, from the generalized homotopy property, this degree should be the degree of $\Phi\left(Z_{1}, \ldots, Z_{n}, R\right)$ with respect to $\Omega_{1} \cap$ $\left\{Z_{n+1}=R\right\}=\varnothing$. Since the set $B \backslash \bar{\Omega}$ is open, there is a path $Z(t)$ from 0 to $(R, 0, \ldots, 0)$ which avoids $\bar{\Omega}$. One may assume that the path is smooth and that $Z_{1}(t) \neq 0$ for $t>0$ (since the set $Z_{1}=0$ is a $(2(n+1)-2$ )surface and the path is one dimensional: use transversality). Since $e^{i \varphi} Z(t)$ does not intersect $\Omega$, one may choose the path such that $Z_{1}(t)$ is real and 
positive for $t>0$. At this point it is not difficult to see that one may choose the path in such a way that it is induced from an equivariant homeomorphism $\Theta\left(\xi_{1}, \ldots, \xi_{n+1}\right)=\left(Z_{1}, \ldots, Z_{n+1}\right)$ such that the $\xi_{1}$-real positive axis is sent into the path (indeed, go to the orbit space where the action is free). Now consider the map

$$
\begin{aligned}
&\left(\left(\left|\xi_{n+1}\right|-a+i\left(\left|\xi_{1}\right|-\left|\xi_{2}\right|\right) \xi_{n+1}^{n_{1} / m_{0}}, \xi_{2}\left(\varepsilon a+\xi_{1} \bar{\xi}_{n+1}\right)\left(R \xi_{n+1}-a \xi_{2}\right)^{n_{2} / m_{0}-1},\right.\right. \\
&\left.\left(R \xi_{n+1}-a \xi_{3}\right)^{n_{3} / m_{0}-1} \xi_{3}, \ldots,\left(R \xi_{n+1}-a \xi_{n}\right)^{n_{n} / m_{0}-1} \xi_{n}\right)
\end{aligned}
$$

for $n>1$, where $(\varepsilon, 0, \ldots, 0, a) \in \Theta^{-1}(\Omega), a>0, \varepsilon$ small enough, and $R$ such that the ball of radius $R$ contains $\Theta^{-1}(\Omega)$. Choose the neighborhood $N$ of $\partial \Omega$ in such a way that $\varphi\left(\xi_{1}, 0, \ldots, 0\right)=1$. The zeros of this map either are $\xi=0$ (where $\varphi=1$ ) or $\left|\xi_{n+1}\right|=a,\left|\xi_{1}\right|=\left|\xi_{2}\right|$, with $\left|\xi_{j}\right|=R$ for some $j$ (hence $\varphi=1$ since one is outside $\Theta^{-1}(\Omega)$ ), or $\xi_{j}=0, j \geq 3$, and $\left|\xi_{1}\right|=0$, or $\varepsilon$ (hence $\varphi=0$ since one is inside $\Theta^{-1}(\Omega)$ ).

For $0 \leq \xi_{n+1} \leq R$, deform the term $\xi_{n+1}^{n_{1} / m_{0}}$ first to 1 , the factors $\left(R \xi_{n+1}-a \xi_{j}\right)$ to $R \xi_{n+1}$ and then to $1(\varphi=1$ on the zeros of this deformation), $\varepsilon a$ to 0 $\left(\varphi=0\right.$ on the zeros of this deformation), and $\xi_{2} \xi_{1} \bar{\xi}_{n+1}$ to $\xi_{2} \xi_{1}$. One obtains the map

$$
\left(2 t+2 \varphi-1, \xi_{n+1}-a+i\left(\left|\xi_{1}\right|-\left|\xi_{2}\right|\right), \xi_{1} \xi_{2}, \xi_{3}, \ldots, \xi_{n}\right)
$$

and, by deforming $\varphi$ to 0 , the $(2 n-2)$-suspension of the Hopf map, if $n>1$. Since the class of $\Theta \circ f$ is the class (in $\mathbf{Z}_{2}$ ) of the map $F$, one has the result in this case.

Now let $l=0, k=0, n=1$ (then $h_{c}=2$ ). If $\Omega$ is such that any map $f\left(Z_{1},\left|Z_{2}\right|\right)$ has a nonzero continuous extension $\tilde{f}$ from $\partial \Omega \cap\left\{0 \leq Z_{2} \leq R\right\}$ to $(\bar{B} \backslash \Omega) \cap\left\{0 \leq Z_{2} \leq R\right\}$, then the class of $(2 t+2 \varphi-1, \tilde{f})$ is the suspension of $\tilde{f}$, i.e., a map from $S^{2}$ into $S^{1}$, hence trivial. For example, if $\Omega=\left\{\left(Z_{1}, Z_{2}\right):\left(\left|Z_{2}\right|-a\right)^{2}+\left|Z_{1}\right|^{2}<r^{2}\right\}, a>r$, one may extend the map $f$ radially outside the ball.

(ii) If $\bar{\Omega} \cap\{Z=0\} \neq \varnothing$, one gets any element of $\operatorname{ker} p_{*}$, except if $l=0$ besides the exceptions already encountered in the previous situations.

For the elements of $\operatorname{Im} p_{*}$, generated by the map

$$
\left(f_{0}\left(x_{0}, t\right), \Phi_{0}\left(x_{0}, t\right), Z_{1}^{n_{1} / m_{0}}, \ldots, Z_{m}^{n_{m} / m_{0}}, 0, \ldots, 0\right)
$$

in the case when $m \leq h_{c}$ and $k \geq l\left(k>l\right.$ if $m=h_{c}$, thus $\left.h_{c} \leq n\right)$, let $\Phi_{0}: \partial\left\{\left\|x_{0}\right\| \leq R_{0}\right\} \rightarrow \mathbf{R}^{l} \backslash\{0\}$ represent any element in $\pi_{k-1}\left(S^{l-1}\right)$. Extend $\Phi_{0}$ to the ball $\left\{\left\|x_{0}\right\| \leq R_{0}\right\}$ radially so that the only zero is at $x_{0}=0$. Thus, the map $\left(\Phi_{0}\left(x_{0}\right), Z_{1}^{n_{1} / m_{0}}, \ldots, Z_{m}^{n_{m} / m_{0}}, 0, \ldots, 0\right)$ has its only zero at the origin and $p_{*}\left(2 t+2 \varphi-1, \Phi_{0}\right)$ is the suspension of the class of the map $\Phi_{0}$. Conversely, if $\Omega$ is such that any nonzero map defined on $\partial(\Omega \cap\{Z=0\})$ extends without zeros to $(\bar{B} \backslash \Omega) \cap\{Z=0\}$, then the $S^{1}$-class of the map $\left(2 t+2 \varphi-1, \Phi_{0}, Z_{1}^{n_{1} / m_{0}}, \ldots, Z_{m}^{n_{m} / m_{0}}, 0, \ldots, 0\right)$ is the suspension of the class of 
$\left(\Phi_{0}, Z_{1}^{n_{1} / m_{0}}, \ldots, Z_{m}^{n_{m} / m_{0}}, 0, \ldots, 0\right)$. Note that if $l=0$, since $\Phi\left(x_{0}, 0\right)=0$, the equivariant map is nonzero on $\partial(\Omega \cap\{Z=0\})$ if $k>0$, but then $\pi_{k}\left(S^{l}\right)=0$.

Finally, if $k=l=0$ then $\operatorname{Im} p_{*}=0$ except if $m<h_{c}$. In fact, in this case $\pi_{0}\left(S^{0}\right)=\mathbf{Z}_{2}$ is generated by $2 t-1$ and then $\left(Z_{1}^{n_{1} / m_{0}}, \ldots, Z^{n_{m} / m_{0}}, 0\right)$ covers $\operatorname{Im} p_{*}$. Q.E.D.

We now give a simple example, as already indicated in the introduction, of a map having trivial generalized degree but its $S^{1}$-degree is nontrivial.

The same example shows that if one looked at the generalized degree on isotropy subgroups, one would not gain more information on the $S^{1}$-degree of the map (unless $m=n$ and the action is the same both on the domain and on the range of $f$ ).

Example D.7. Let $D$ be the unit disk in $\mathbf{C}^{3}$ and let $f: D \rightarrow \mathbf{R} \times \mathbf{C}^{2}$ be defined by

$$
f\left(\lambda, z_{1}, z_{2}\right)=\left(\left|z_{1}\right|^{2}+\left|z_{2}\right|^{2}-\frac{1}{2}, \lambda z_{1}, \lambda^{\alpha} z_{2}\right) ;
$$

if $\alpha$ is negative then $\lambda^{\alpha}=\bar{\lambda}^{|\alpha|}$.

The map $f$ has generalized degree 0 in $\pi_{5}\left(S^{4}\right)$ if and only if $\alpha$ is odd (see $\left[\mathrm{I}_{0}\right.$, p. 9]).

In particular, if $\alpha=1$ the map

$$
\tilde{f}\left(\lambda, z_{1}, z_{2}\right)=\left(\left|z_{1}\right|^{2}+\left|z_{2}\right|^{2}-\frac{1}{2}, \lambda z_{1}-\kappa\left(\lambda, z_{1}, z_{2}\right) \bar{z}_{2}, \lambda z_{2}+\kappa\left(\lambda, z_{1}, z_{2}\right) \bar{z}_{1}\right) \text {, }
$$

where $\kappa\left(\lambda, z_{1}, z_{2}\right)=1-\left(|\lambda|^{2}+\left|z_{1}\right|^{2}+\left|z_{2}\right|^{2}\right)$, is a nonvanishing extension of $f_{\mid \partial D}$ to $D$.

Now if we take on both $\mathbf{C}^{3}$ and $\mathbf{R} \times \mathbf{C}^{2}$ the standard semifree $S^{1}$-actions

$$
\begin{gathered}
\mathbf{e}^{i \varphi}\left(\lambda, z_{1}, z_{2}\right)=\left(\lambda, e^{i \varphi} z_{1}, e^{i \varphi} z_{2}\right), \quad\left(\lambda, z_{1}, z_{2}\right) \in \mathbf{C}^{3}, \\
\mathbf{e}^{i \varphi}\left(y_{0}, \xi_{1}, \xi_{2}\right)=\left(y_{0}, e^{i \varphi} \xi_{1}, e^{i \varphi} \xi_{2}\right), \quad\left(y_{0}, z_{1}, z_{2}\right) \in \mathbf{R} \times \mathbf{C}^{2},
\end{gathered}
$$

then the map $f$ is $S^{1}$-equivariant and has $S^{1}$-degree the couple $(0, \alpha+1)$, where 0 corresponds to the degree of the invariant part $f(\lambda, 0,0)=-\frac{1}{2}$ and $\alpha+1$ comes from its equivariant part. To see this, use the $S^{1}$-homotopy

$$
\left(\left|z_{1}\right|^{2}+\left|z_{2}\right|^{2}-\frac{1}{2},\left(\begin{array}{cc}
(1-t) \lambda & -t \\
t \lambda^{\alpha+1} & (1-t) \lambda^{\alpha}
\end{array}\right)\left(\begin{array}{l}
z_{1} \\
z_{2}
\end{array}\right)\right) .
$$

Hence, $\alpha+1$ is the Brouwer degree of the map $\left(\left|z_{1}\right|^{2}+\left|z_{2}\right|^{2}-\frac{1}{2}, \lambda^{\alpha+1} z_{1}, z_{2}\right)$ with respect to the open set $\left\{\left(\lambda, z_{1}, z_{2}\right) \in D, z_{1} \in \mathbf{R}^{+}\right\}$.

Thus the $S^{1}$-degree of $f$ is nontrivial if and only if $\alpha \neq-1$. In the case when $\alpha=-1$, the map $\tilde{f}\left(\lambda, z_{1}, z_{2}\right)=\left(\left|z_{1}\right|^{2}+\left|z_{2}\right|^{2}-\frac{1}{2}, \lambda z_{1}-\kappa\left(\lambda, z_{1}, z_{2}\right) z_{2}, \bar{\lambda} z_{2}+\right.$ $\left.\kappa\left(\lambda, z_{1}, z_{2}\right) z_{1}\right)$ is a nonvanishing $S^{1}$-equivariant extension of $f$ to $D$.

Observe that the degrees of the map $f$ on the isotropy subspaces do not give any further information. Indeed, since the above $S^{1}$-action is semifree the only 
isotropy subgroups $H$ of $S^{1}$ are $\{e\}$ and $S^{1}$ itself. If $H=\{e\}$, then the degree of $f$ is nontrivial only if $\alpha$ is even and, if $H=S^{1}$, then one is left with only the invariant part of $f$.

If instead we now let $S^{1}$ act on both $\mathbf{C}^{3}$ and $\mathbf{R} \times \mathbf{C}^{2}$ as

$$
\begin{aligned}
\mathbf{e}^{i \varphi}\left(\lambda, z_{1}, z_{2}\right) & =\left(\lambda, e^{i \varphi} z_{1}, e^{i 2 \varphi} z_{2}\right), \\
\mathbf{e}^{i \varphi}\left(y_{0}, \xi_{1}, \xi_{2}\right) & =\left(y_{0}, e^{i \varphi} \xi_{1}, e^{i 2 \varphi} \xi_{2}\right),
\end{aligned}
$$

we may reduce the analysis to a $S^{1}$-almost semifree action by considering the map $\tilde{f}: \mathbf{C}^{3} \rightarrow \mathbf{R} \times \mathbf{C}^{2}$ defined by

$$
\tilde{f}\left(\lambda, Z_{1}, Z_{2}\right)=f\left(\lambda, Z_{1}, Z_{2}^{2}\right)=\left(\left|Z_{1}\right|^{2}+\left|Z_{2}^{2}\right|^{2}-\frac{1}{2}, \lambda Z_{1}, \lambda^{\alpha} Z_{2}^{2}\right),
$$

where $\mathbf{C}^{3}$. denotes $\mathbf{C}^{3}$ with the standard semifree $S^{1}$-action. Now the map $\tilde{f}$ has $S^{1}$-degree the couple $(0,2+\alpha)$. To show this, we may proceed as follows. To each map of the form $\left(f_{0}, f_{1}, f_{2}\right): \mathbf{C}^{3} \rightarrow \mathbf{R} \times \mathbf{C}^{2}$ we associate the $S^{1}$-equivariant map $\left(f_{0}, f_{1}^{2}, f_{2}\right): \mathbf{C}^{3} \rightarrow \mathbf{R} \times \mathbf{C}_{\diamond}^{2}$, where $\mathbf{R} \times \mathbf{C}_{\diamond}^{2}$ stands for $\mathbf{R} \times \mathbf{C}^{2}$ with the $S^{1}$-action $\left(y_{0}, e^{i 2 \varphi} \xi_{1}, e^{i 2 \varphi} \xi_{2}\right)$.

This operation induces a multiplication by 2 for the $S^{1}$-degree of the map

$$
\left(\left|Z_{1}\right|^{2}+\left|Z_{2}\right|^{2}-\frac{1}{2}, \lambda^{2} Z_{1}^{2}, \lambda^{\alpha} Z_{2}\right)
$$

from $\mathbf{C}^{3}$. into $\mathbf{R} \times \mathbf{C}^{2}$ with the standard $S^{1}$-action. Using the same argument as above, we obtain that the map

$$
\left(\left|Z_{1}\right|^{2}+\left|Z_{2}^{2}\right|^{2}-\frac{1}{2}, \lambda^{2} Z_{1}^{2}, \lambda^{\alpha} Z_{2}^{2}\right)
$$

has $S^{1}$-degree equal to $(0,2(2+\alpha))$ and therefore our original map has $S^{1}$ degree equal to $(0,2+\alpha)$.

If $\alpha=-2$, the map

$$
\left(\left|Z_{1}\right|^{2}+\left|Z_{2}^{2}\right|^{2}-\frac{1}{2}, \lambda Z_{1}-\kappa\left(\lambda, Z_{1}, Z_{2}\right) Z_{2}, \bar{\lambda}^{2} Z_{2}^{2}+\kappa\left(\lambda, Z_{1}, Z_{2}\right)^{2} Z_{1}^{2}\right)
$$

is a nonvanishing $S^{1}$-equivariant extension of $\tilde{f}$ but it does not arise from a map depending on the variables $\left(\lambda, z_{1}, z_{2}\right)$. In fact, the map $\left(\left|z_{1}\right|^{2}+\left|z_{2}\right|^{2}-\right.$ $\left.\frac{1}{2}, \lambda z_{1}, \lambda^{\alpha} z_{2}\right)$, with the $S^{1}$-action given by $(*)$, has $S^{1}$-degree $(0, \alpha, 2+\alpha)$ which is never vanishing. This computation will be undertaken in [I.M.V.1].

We add a final warning: in general the $\Gamma$-degree of a map will have several components (as in the last case) corresponding to different isotropy subspaces, but the fact that one of these components is nonzero does not imply the existence of zeros in the corresponding isotropy subspace, as the following example shows.

Consider on both $\mathbf{R} \times \mathbf{C}^{2}$ and $\mathbf{R} \times \mathbf{R} \times \mathbf{C}$ the $S^{1}$-action given by

$$
\begin{aligned}
\mathbf{e}^{i \varphi}\left(x_{0}, \lambda, z\right) & =\left(x_{0}, \lambda, e^{i \varphi} z\right), \\
\mathbf{e}^{i \varphi}\left(y_{1}, y_{2}, \xi\right) & =\left(y_{1}, y_{2}, e^{i \varphi} \xi\right) .
\end{aligned}
$$

The $S^{1}$-equivariant map

$$
f\left(x_{0}, \lambda, z\right)=\left(x_{0}^{2}+|z|^{2}-\frac{1}{2},|\lambda|^{2} x_{0}+|z|^{2}, \lambda z\right),
$$


from the unit disk $D$ in $\mathbf{R} \times \mathbf{C}^{2}$ into $\mathbf{R} \times \mathbf{R} \times \mathbf{C}$ has the invariant part

$$
f\left(x_{0}, \lambda, 0\right)=\left(x_{0}^{2}-\frac{1}{2},|\lambda|^{2} x_{0}\right)
$$

with trivial generalized degree in $\pi_{3}\left(S^{2}\right)$. In fact, it has a nonvanishing extension to $D$ given by

$$
\left(x_{0}^{2}-\frac{1}{2},|\lambda|^{2} x_{0}+\left(1-x_{0}^{2}-|\lambda|^{2}\right) x_{0}\right) .
$$

On the other hand, the other component of the $S^{1}$-degree of $f$ is given by the Brouwer degree of the map

$$
\left(x_{0}^{2}+|z|^{2}-\frac{1}{2},|\lambda|^{2} x_{0}+\left(1-x_{0}^{2}-|\lambda|^{2}-|z|^{2}\right) x_{0}+|z|^{2}, \lambda z\right)
$$

with respect to the set $\left\{x_{0}^{2}+|\lambda|^{2}+|z|^{2} \leq 1, z \in \mathbf{R}^{+}\right\}$. Since the only zero of this map is $\left(x_{0}=-\frac{1}{2}, \lambda=0, z=\frac{1}{2}\right)$, it is easy to see that its degree is 1 . Thus the map $f$ has nontrivial $S^{1}$-degree arising from its $S^{1}$-equivariant component, but the only zeros of $f$ are $\left(x_{0}^{2}=\frac{1}{2}, \lambda=0, z=0\right)$; i.e., they belong to the fixed-point subspace of stationary points.

\section{REFERENCES}

[A] J. F. Adams, Prerequisites (on equivariant stable homotopy theory) for Carlsson's lecture. Lecture Notes in Math., vol. 1051, Algebraic Topology, Aarhus, Springer-Verlag, Berlin, 1982, pp. 483-532.

[B] G. E. Bredon, Introduction to compact transformation groups, Academic Press, New York, 1972.

[C.M] Group actions on manifolds, Contemp. Math., vol. 36, Amer. Math. Soc., Providence, R. I., 1985.

[C.M.Y] S. N. Chow, J. Mallet-Paret, and J. A. Yorke, Global Hopf bifurcation from a multiple eigenvalue, Nonlinear Anal. M.T.A. 2 (1978), 755-763.

[Da] E. N. Dancer, $A$ new degree for $S^{1}$-invariant gradient mappings and applications, Ann. Inst. H. Poincaré Anal. Non Linéaire 2 (1985), 329-370.

[Da.1] _ Perturbation of zeros in the presence of symmetries, J. Austral. Math. Soc. (Ser. A) 36 (1984), 106-125.

[D] J. Dugundji, Topology, Allyn \& Bacon, Boston, Mass., 1966.

[F] F. B. Fuller, An index of fixed point type for periodic orbits, Amer. J. Math, 89 (1967), 133-148.

[G.M.V] K. Geba, I. Massabó, and A. Vignoli, Generalized topological degree and bifurcation, Nonlinear Functional Analysis and Its Applications (Proc. NATO Advanced Study Inst., Maratea, Italy, 1985), Mathematical and Physical Sciences, vol. 173, Reidel, Dordrecht, 1986, pp. 5473.

[G] G. Greenberg, Lectures on algebraic topology, Benjamin, New York, 1967.

[H] H. Hauschild, Äquivariante Homotopie, I, Arch. Math. 29 (1977), 158-167.

[HI] __ Zerspaltung äquivarianter Homotopiemengen, Math. Ann. 230 (1977), 279-292.

[K] C. Kosniowski, Equivariant cohomology and stable cohomotopy, Math. Ann. 210 (1974), 83104.

[ $\left.\mathrm{I}_{0}\right]$ J. Ize, Bifurcation theory for Fredholm operators, Mem. Amer. Math. Soc. (7) 174 (1976).

[I] - Obstruction theory and multiparameter Hopf bifurcation, Trans. Amer. Math. Soc. 289 (1985), 757-792. 
[I.M.P.V] J. Ize, I. Massabó, J. Pejsachowicz, and A. Vignoli, Structure and dimension of global branches of solutions to multiparameter nonlinear equations, Trans. Amer. Math. Soc. 291 (1985), 383-435.

[I.M.V] J. Ize, I. Massabó, and A. Vignoli, Global results on continuation and bifurcation for equivariant maps, Nonlinear Functional Analysis and Its Applications, (Proc. NATO Advanced Study Inst., Maratea, Italy, 1985), Mathematical and Physical Sciences, vol. 173, Reidel, Dordrecht, 1986, pp. 74-111.

[I.M.V.1] _ Degree theory for equivariant maps, II (in preparation).

[L.S] J. Leray and J. Schauder, Topologie et équations fonctionelles, Ann. Sci. École Norm. Sup. 51 (1934), 45-78.

[Na] U. Namboodiri, Equivariant vector fields on spheres, Trans. Amer. Math. Soc. 278 (1983), 431-460.

[N] L. Nirenberg, Comments on nonlinear problems, Le Matematiche 36 (1981), 109-119.

[R] W. Rudin, Functional analysis, McGraw-Hill, New York, 1973.

[S] E. Spanier, Algebraic topology, McGraw-Hill, New York, 1966.

[t.D] T. tom Dieck, Transformation groups and representation theory, Lecture Notes in Math., vol. 766, Springer-Verlag, New York, 1979.

[V] A. Vanderbauwhede, Local bifurcation and symmetry, Res. Notes in Math., Pitman, Boston, Mass., 1982.

[W] G. W. Whitehead, Elements of homotopy theory, Graduate Texts in Math., Springer-Verlag, New York, 1978.

IIMAS-Unam, Apartado Postal 20-726, Administracion 20, Delegacion Alvaro ObreGón, 01000 Mexico D.F., MeXico

Dipartimento di Matematica, Università di Roma Tor Vergata, Via Orazio Raimondo (La Romanina), 00173 Rome, Italy

Dipartimento di Matematica, Università della Calabria, 87036 Arcavacata Dirende (CS), ITALY 